\title{
A Decade of Progress in Deep Brain Stimulation of the Subcallosal Cingulate for the Treatment of Depression
}

\author{
Sharafuddin Khairuddin ${ }^{1,+}$, Fung Yin Ngo ${ }^{1,+}$, Wei Ling Lim ${ }^{1,2}{ }^{1}$, Luca Aquili ${ }^{3}{ }^{(D}$, \\ Naveed Ahmed Khan ${ }^{4}{ }^{\oplus}$, Man-Lung Fung ${ }^{1}$, Ying-Shing Chan ${ }^{1}$, Yasin Temel ${ }^{5}$ \\ and Lee Wei $\operatorname{Lim} 1,2, *$ (D) \\ 1 Neuromodulation Laboratory, School of Biomedical Sciences, Li Ka Shing Faculty of Medicine, \\ The University of Hong Kong, L4 Laboratory Block, 21 Sassoon Road, Hong Kong, China; \\ sharaf@hku.hk (S.K.); fyngo1@connect.hku.hk (F.Y.N.); weilingl@sunway.edu.my (W.L.L.); \\ fungml@hku.hk (M.-L.F.); yschan@hku.hk (Y.-S.C.) \\ 2 Department of Biological Sciences, School of Science and Technology, Sunway University, \\ Bandar Sunway 47500, Malaysia \\ 3 School of Psychological and Clinical Sciences, Charles Darwin University, NT0815 Darwin, Australia; \\ luca.aquili@cdu.edu.au \\ 4 Department of Biology, Chemistry and Environmental Sciences, College of Arts and Sciences, \\ American University of Sharjah, Sharjah 26666, UAE; naveed5438@gmail.com \\ 5 Departments of Neuroscience and Neurosurgery, Maastricht University, 6229ER Maastricht, \\ The Netherlands; y.temel@maastrichtuniversity.nl \\ * Correspondence: drlimleewei@gmail.com \\ + These authors have joint first authorship.
}

Received: 14 August 2020; Accepted: 28 September 2020; Published: 12 October 2020

\begin{abstract}
Major depression contributes significantly to the global disability burden. Since the first clinical study of deep brain stimulation (DBS), over 446 patients with depression have now undergone this neuromodulation therapy, and 29 animal studies have investigated the efficacy of subgenual cingulate DBS for depression. In this review, we aim to provide a comprehensive overview of the progress of DBS of the subcallosal cingulate in humans and the medial prefrontal cortex, its rodent homolog. For preclinical animal studies, we discuss the various antidepressant-like behaviors induced by medial prefrontal cortex DBS and examine the possible mechanisms including neuroplasticity-dependent/independent cellular and molecular changes. Interestingly, the response rate of subcallosal cingulate Deep brain stimulation marks a milestone in the treatment of depression. DBS achieved response and remission rates of $64-76 \%$ and $37-63 \%$, respectively, from clinical studies monitoring patients from 6-24 months. Although some studies showed its stimulation efficacy was limited, it still holds great promise as a therapy for patients with treatment-resistant depression. Overall, further research is still needed, including more credible clinical research, preclinical mechanistic studies, precise selection of patients, and customized electrical stimulation paradigms.
\end{abstract}

Keywords: deep brain stimulation; treatment-resistant depression; major depressive disorder; subcallosal cingulate; medial prefrontal cortex

\section{Introduction}

Major depressive disorder (MDD) contributes significantly to the global disability burden and social burden [1,2]. In the US from 2005 to 2010, the economic burden of patients with major depressive 
disorder increased by $21.5 \%$ to $\$ 210.5$ billion [3]. The main symptoms of MDD include severe sadness, anxiety, cognitive deterioration, and suicidal thoughts [4]. Although its etiology is uncertain, genetic predisposition, developmental deficits, hormonal imbalance, and a stressful lifestyle may increase the risk for MDD [5-10].

Prior to the discovery of antidepressant medication, surgical ablation was used to effectively treat MDD in the US and Europe [11]. Pharmacological antidepressants first appeared in the late 20th century and these first-generation drugs became the first line treatment for depression [12]. However, newer generations of antidepressants were barely more effective than first-generation tricyclic antidepressants [13] and this has led to the emergence of treatment resistance. Treatment-resistant depression (TRD) is the failure to respond to the three different classes of treatment: antidepressants, psychotherapy, or electroconvulsive therapy given at a sufficient dose and time [14,15]. Approximately $20 \%$ to $30 \%$ of patients are refractory to pharmacotherapy and nearly $60 \%$ respond inadequately $[16,17]$, which can result in worse clinical responses, leading to additional social burdens [18]. As the pathogenesis of MDD involves multiple structures, a broad-acting safe therapy needs to be developed $[19,20]$.

With much progress in surgical techniques and advances in cardiac pacemakers, electrical stimulation has matured to become an adjustable stimulatory regimen [21]. Deep brain stimulation (DBS) is a procedure whereby deep brain structures are stimulated via precisely implanted electrodes. It was first used to alleviate movement disorders in patients with Parkinson's disease [22]. With advances in our understanding of the limbic circuitry, the focus has shifted to the antidepressant-like effects of DBS [23]. Some recent clinical studies have shown that DBS holds great promise in treating patients with TRD, and mechanistic studies in animals are currently in progress.

The use of DBS as a treatment for TRD was first proposed in a study by Kruger et al. on the differences in regional cerebral blood flow ( $\mathrm{rCBF}$ ) between remitted patients and bipolar depression (BD) patients [24]. They observed that rCBF in Brodmann Area 25 (BA25) was higher in remitted and BD patients compared to control patients and this was also seen in healthy patients with self-rated high negative affect [25]. Furthermore, Kruger et al. noted that mood provocation did not change the $\mathrm{rCBF}$ to this region in $\mathrm{BD}$ patients compared to MDD patients, indicating that dysfunction in the region was specific to depression [24]. Mayberg et al., who are pioneers of DBS as a treatment for depression, subsequently targeted BA25 after detecting metabolic abnormalities within the region that were consistent with those found in patients with TRD [19]. This landmark paper led to further developments in the application of DBS of this region as a treatment for depression.

Indeed, several research groups have used DBS to treat depression by targeting different brain regions in the limbic system. Jimenez et al. applied DBS to the inferior thalamic peduncle, whereas Schlaepfer et al. applied DBS in the nucleus accumbens core $[26,27]$ and successfully performed DBS on the medial forebrain bundle [28]. With rapid developments in DBS as a treatment for TRD, research is now focusing on the subcallosal cingulate (SCC). This review aims to examine and consolidate clinical and preclinical research on the use of DBS as a treatment for depression, targeting the subcallosal cingulate in humans and the ventromedial prefrontal cortex, the anatomical correlate in rodents.

\section{Outline of the Review}

The online PubMed database was searched for research articles in English using a Boolean operation with keywords including "deep brain stimulation" AND "depression" AND "subcallosal cingulate" OR "rodent" AND "medial prefrontal cortex". Relevant articles cited in the reference lists of the identified publications were also included. PubMed was utilized due to its extensive collection of indexed peer-reviewed journals. This review highlights the development of DBS as a treatment for TRD and discusses the findings and limitations of preclinical and clinical studies published in the recent decade. The neuroplasticity-dependent and -independent aspects of the molecular and 
cellular changes due to DBS are also discussed. Lastly, some potential approaches that may improve the precision, safety, and efficacy of DBS are proposed.

\section{The Development of Deep Brain Stimulation as a Treatment for Depression}

Deep brain stimulation involves the stereotactic implantation of thin electrodes in deep brain structures that are used to deliver electrical stimulation generated by a subcutaneous pulse generator $[29,30]$. Stimulation is generally applied at either a low/moderate frequency $(5-90 \mathrm{~Hz})$ or high frequency $(100-400 \mathrm{~Hz})$. Since the inception of DBS, a number of studies have demonstrated that this modality has the ability to treat pain, obsessive-compulsive disorder, and Parkinson's disease [16,21,31]. Its efficacy has been verified in Parkinson's disease patients, in which high frequency stimulation (HFS) of specific brain region(s) in the basal ganglia was able to stop tremors [21,32]. The use of DBS has been given FDA approval for the management of obsessive-compulsive disorder since 2007, but it is only provided under a humanitarian device exemption [33,34].

The following sections summarize the clinical studies on deep brain stimulation in the subcallosal cingulate for treating patients with treatment-resistant depression and preclinical studies of deep brain stimulation in the medial prefrontal cortex (mPFC) of rodents.

\section{Clinical and Preclinical Studies of SCC DBS for the Treatment of Depression}

Clinical studies of depression utilize rating scales of depression that assess changes in depressive symptoms in patients. Some scales are completed by the researcher such as the Hamilton Depression Rating Scale (HDRS) and the Montgomery-Åsberg Depression Rating Scale (MADRS). These rating scales should allow more consistent assessment between patients, but can lack consensus in their interpretation among researchers, which could lead to misdiagnosis [35]. Another weakness of rating scales conducted in this manner is that the accuracy of the results is dependent on the communication skills of the patient, which might be hampered by the disease itself. Other scales are completed by patients such as the Beck Anxiety Inventory (BAI), Beck Depression Inventory (BDI), and Quick Inventory of Depressive Symptomatology (QIDS). These rating scales should allow for a more accurate reporting of depressive symptoms, although the number and/or depth of questions may vary across different tests.

\subsection{Progress in the Development of SCC-DBS}

Different papers have referred to the SCC and similar regions under different names, e.g., the subcallosal cingulate gyrus (SCG), the subgenual cingulate, as well as Brodmann areas. Different historical names allow for different historical context. The subgenual cortex is used more interchangeably with the term Brodmann Area 25, named after Korbinian Brodmann. The subgenual cortex is located in the cingulate region as a narrow band in the caudal portion of the subcallosal area adjacent to the paraterminal gyrus. By comparison, the SCG is comprised of Brodmann areas 25, 24, and 32 [36]; SCG circuits; and limbic structures. The SCG is pivotal in mood, learning, reward, and memory [37] and has been implicated as an aberrant region in MDD. As the SCC can be effectively targeted by antidepressants, this makes the SCG a potential target of DBS against TRD [38,39]. Tables 1 and 2 list 39 clinical studies on the treatment efficacy of SCC-DBS for TRD. 
Table 1. A list of clinical studies on deep brain stimulation of the human subcallosal cingulate.

\begin{tabular}{|c|c|c|c|c|c|c|c|}
\hline Authors & Main Inclusion Criteria & No. of Patients & $\begin{array}{c}\text { Stimulation Target \& DBS } \\
\text { Design }\end{array}$ & Stimulation Parameters & $\begin{array}{c}\text { Clinical } \\
\text { Evaluation }\end{array}$ & Major Outcomes & Adverse Effects \\
\hline Sankar et al. $2020[40]$ & $\begin{array}{l}\text { TRD MDE; } \\
\text { Current MDE } \geq 12 \text { months; } \\
\text { HRSD-17 score } \geq 20 ; \\
\text { Non-responsive (NR) } \geq 4 \\
\text { antidepressant therapies; } \\
\text { HRSD-17 score } \geq 20 \text {. }\end{array}$ & 27 & $\underline{\text { Target SCG }}$ & $\begin{array}{c}\frac{\frac{\text { Implantation }}{\text { Bilateral }}}{\text { Stimulation Monopolar }} \\
\frac{\text { Frequency }}{130 \mathrm{~Hz}} \\
\frac{\text { Amplitude }}{3-6 \mathrm{~V}} \\
\frac{\text { Pulse Width }}{90 \mu \mathrm{s}}\end{array}$ & $\begin{array}{c}\text { Volumetric } \\
\text { analysis, } \\
\text { Whole brain grey } \\
\text { and white matter } \\
\text { analysis }\end{array}$ & $\begin{array}{l}\text { Left and average SCG volume } \\
\text { significantly higher in responders } \\
\text { compared to non-responders. } \\
\text { Right and average amygdala } \\
\text { volume significantly higher in } \\
\text { responders compared to } \\
\text { non-responders. } \\
\text { Left, right, and average thalamus } \\
\text { volume significantly higher in } \\
\text { responders compared to } \\
\text { non-responders. } \\
\text { Brain grey matter volume } \\
\text { significantly lower in responders } \\
\text { compared to non-responders. } \\
\text { Ratio of grey to white matter } \\
\text { volume significantly higher in } \\
\text { responders compared to } \\
\text { non-responders. }\end{array}$ & N.A. \\
\hline Riva-Posse et al. (2019) [41] & $\begin{array}{c}\text { TRD MDE; } \\
\text { Current MDE } \geq 12 \text { months; } \\
\text { HRSD-17 score } \geq 20 ; \\
\text { Non-responsive (NR) } \geq 4 \\
\text { antidepressant therapies; } \\
\text { HRSD-17 score } \geq 20 ; \\
\text { GAF }<50 \text {. }\end{array}$ & 9 & $\begin{array}{l}\text { Target SCC } \\
\text { Study Design Intraoperative } \\
\text { Sessions: } 6 \text { min session of } 3 \mathrm{~min} \\
\text { stimulation ON and } 3 \mathrm{~min} \\
\text { stimulation OFF. } \\
\text { Number of sessions: } \\
12 \text { trials (one at each of the eight } \\
\text { available contacts. Four per } \\
\text { hemisphere, plus four } \\
\text { sham trials). } \\
\text { Sham-controlled, double-blinded } \\
\text { trials (one case w/single } \\
\text { blind trial) }\end{array}$ & $\begin{array}{c}\frac{\begin{array}{c}\text { Implantation } \\
\text { Bilateral }\end{array}}{\text { Stimulation Monopolar }} \\
\frac{\text { Frequency }}{130 \mathrm{~Hz}} \\
\frac{\text { Amplitude }}{6 \mathrm{~mA}} \\
\frac{\text { Pulse Width }}{90 \mu \mathrm{s}}\end{array}$ & $\begin{array}{c}\text { ECG, EDA, MRI } \\
\text { Volume of Tissue } \\
\text { Activated, } \\
\text { Structural } \\
\text { Connectivity } \\
\text { Analysis }\end{array}$ & $\begin{array}{l}\text { Autonomic changes with SCC-DBS } \\
\text { correspond to salient behavioral } \\
\text { responses. } \\
\text { Distant effects of SCC-DBS in the } \\
\text { midcingulate cortex. } \\
\text { Increase in heart rate was only seen } \\
\text { with left SCC-DBS. } \\
\text { No significant relationship with } \\
\text { skin conductance. } \\
\text { These findings aid in the optimal } \\
\text { selection of contacts and } \\
\text { parameters in SCC-DBS surgery. }\end{array}$ & N.A. \\
\hline Eitan et al. (2018) [42] & $\begin{array}{c}\text { Both sexes, } 21-70 \text { years; } \\
\text { Non-psychotic MDD; } \\
\text { First MDE onset before } 45 \\
\text { years old with current MDE } \\
\geq 12 \text { months; } \\
\text { NR } \geq 4 \text { antidepressant } \\
\text { therapies; MADRS } \geq 22 ; \\
\text { GAF }<50 \text {; MMSE }>24 ; \\
\text { No changes in current } \\
\text { antidepressant treatments } \geq \\
4 \text { wks prior to study. }\end{array}$ & 9 & $\begin{array}{c}\text { Target BA25 } \\
\text { Study Design } \\
\text { Double-blind, randomized. } \\
\text { Two groups: High- OR } \\
\text { low-frequency DBS for } 12 \text { months } \\
\text { from } 1 \text { month after electrode } \\
\text { implantation. }\end{array}$ & $\begin{array}{l}\text { Implantation } \\
\text { Bilateral Stimulation } \\
\text { Monopolar } \\
\text { Frequency } \\
20 \mathrm{~Hz} \text { or } 130 \mathrm{~Hz} \\
\frac{\text { Amplitude }}{4-8 \mathrm{~mA}} \\
\frac{\text { Pulse Width }}{91 \mu \mathrm{s}}\end{array}$ & $\begin{array}{c}\text { MADRS, HRSD-17, } \\
\text { QIDS-SR, } \\
\text { Q-LES-Q, GAF, } \\
\text { HAM-A, CGI, PGI, } \\
\text { CANTAB battery. }\end{array}$ & $\begin{array}{l}\text { Four out of nine patients } \\
\text { responded }{ }^{\diamond} \text { at the end of DBS } \\
\text { ( } \geq 40 \% \text { reduction in MADRS from } \\
\text { baseline). } \\
\text { The effect of DBS at 6-12 months } \\
\text { was higher than DBS at } 1-6 \text { months. } \\
\text { High-frequency DBS showed } \\
\text { higher efficacy than } \\
\text { low-frequency DBS. } \\
\text { Non-responders crossed over after } \\
\text { first } 6 \text { months of DBS. }\end{array}$ & $\begin{array}{l}\text { Severe } \\
\text { One patient overdosed on } \\
\text { medication (dothiepin and } \\
\text { valium). }\end{array}$ \\
\hline
\end{tabular}


Table 1. Cont

\begin{tabular}{|c|c|c|c|c|c|c|c|}
\hline Authors & Main Inclusion Criteria & No. of Patients & $\begin{array}{c}\text { Stimulation Target \& DBS } \\
\text { Design }\end{array}$ & Stimulation Parameters & $\begin{array}{c}\text { Clinical } \\
\text { Evaluation }\end{array}$ & Major Outcomes & Adverse Effects \\
\hline Merkl et al. (2018) [43] & $\begin{array}{l}\text { Diagnosed MDD and } \\
\text { disease lasted for }>2 \text { years; } \\
\text { HAMD-24 score } \geq 20 ; \\
\text { ATHF Score } \geq 3 ; \\
\text { TRD: NR } \geq 2 \text { antidepressant } \\
\text { therapies; } \\
\text { Failed to respond to } \\
\text { antidepressants and ECT; } \\
\text { No changes in current } \\
\text { antidepressant treatments } \\
\geq 6 \text { wks prior to study. }\end{array}$ & 8 & $\begin{array}{l}\text { Target SCG } \\
\frac{\text { Study Design }}{\text { Randomized; }} \\
\text { Two groups: sham-DBS (delayed } \\
\text { onset) OR non-delayed onset } \\
\text { group for the first 8 weeks in a } \\
\text { blinded manner. } \\
\text { Open-label DBS afterwards for up } \\
\text { to } 28 \text { months. }\end{array}$ & $\begin{array}{l}\frac{\text { Implantation }}{\text { Bilateral }} \\
\frac{\text { Stimulation }}{\text { Monopolar }} \\
\frac{\text { Frequency }}{130 \mathrm{~Hz}} \\
\frac{\text { Amplitude }}{5-7 \mathrm{~V}} \\
\frac{\text { Pulse Width }}{90 \mu \mathrm{s}}\end{array}$ & $\begin{array}{l}\text { HAMD-24, } \\
\text { BDI, } \\
\text { MADRS. }\end{array}$ & $\begin{array}{l}\text { Three out of eight patients } \\
\text { responded ** after } 6 \text { months DBS; } \\
\text { three out of seven with the same } \\
\text { criteria after } 12 \text { months. } \\
\text { Two out of six responded ** at the } \\
\text { end of DBS, follow-up at } 28 \text { months. } \\
\text { Two out of six patients reached } \\
\text { remission } \square \text { at the end of DBS, } \\
\text { follow-up at } 28 \text { months. } \\
\text { This study showed a delayed } \\
\text { response in patients; no significant } \\
\text { antidepressant effects between } \\
\text { sham and active stimulation } \\
\text { compared to baseline. }\end{array}$ & $\begin{array}{c}\text { Non-severe } \\
\text { Headach; } \\
\text { Pain; } \\
\text { Scalp tingling; } \\
\text { Dizziness; } \\
\text { Light hypomania; } \\
\text { Inconvenient movement; } \\
\frac{\text { Severe }}{\text { NIL }}\end{array}$ \\
\hline Howell et al. (2018) [44] & $\begin{array}{c}\text { Both sexes aged } 18-70 \text { years; } \\
\text { current MDE } \geq 12 \text { months; } \\
\text { NR } \geq 4 \\
\text { antidepressant therapies; } \\
\text { HRSD-17 score } \geq 20 \text {; } \\
\text { GAF score } \leq 50\end{array}$ & 6 & $\begin{array}{l}\frac{\text { Target SCC (Cingulum Bundle }}{\text { and Forceps Minor) }}\end{array}$ & $\begin{array}{l}\frac{\text { Implantation }}{\text { Bilateral }} \\
\frac{\text { Stimulation }}{\text { Monopolar }} \\
\frac{\text { Frequency }}{130 \mathrm{~Hz}} \\
\frac{\text { Amplitude }}{4 \mathrm{~V}} \\
\frac{\text { Pulse Width }}{90 \mu \mathrm{s}}\end{array}$ & $\begin{array}{l}\text { HDRS-17, MRI } \\
\text { Field-cable } \\
\text { modeling } \\
\text { (no-VTA-based } \\
\text { analysis) }\end{array}$ & $\begin{array}{l}\text { All of the subjects responded. } \\
\text { Left and right cingulum bundles as } \\
\text { well as forceps minor are the most } \\
\text { likely therapeutic targets. } \\
\text { Right cingulum bundle activation } \\
\text { beyond a threshold may } \\
\text { protract recovery. } \\
\text { Uncinate fasciculus and frontal } \\
\text { pole were activated to a lesser } \\
\text { extent, may not be necessary for } \\
\text { anti-depressive effect of SCC-DBS. } \\
\text { Time to a atable response (TSR) was } \\
\text { 8-189 days, } 1 \text {-year HDRS-17 } \\
\text { was 2-11. } \\
\text { Field cable modeling was more } \\
\text { accurate than volume of activated } \\
\text { tissue at approximating } \\
\text { axonal activation. } \\
\text { Overstimulation of CB-DBS can be } \\
\text { detrimental to the recovery process. }\end{array}$ & N.A. \\
\hline Waters et al. (2018) [45] & $\begin{array}{c}\text { Both sexes aged } 18-70 \text { years; } \\
\text { current MDE } \geq 12 \text { months; } \\
\text { NR } \geq 4 \\
\text { antidepressant therapies; } \\
\text { HRSD-17 score } \geq 20 ; \\
\text { GAF score } \leq 50\end{array}$ & 4 & $\begin{array}{l}\text { Target SCC } \\
\text { Study Design } \\
\begin{array}{c}\text { Single-blinded, } \\
\frac{\text { Session }}{3 \mathrm{~min}}\end{array}\end{array}$ & $\begin{array}{l}\frac{\text { Implantation }}{\text { Bilateral }} \\
\text { Stimulation } \\
\text { Monopolar } \\
\frac{\text { Frequency }}{130 \mathrm{~Hz}} \\
\frac{\text { Amplitude }}{3-5 \mathrm{~V}} \\
\frac{\text { Pulse Width }}{90 \mu \mathrm{s}}\end{array}$ & $\begin{array}{l}\text { HDRS-17, } \\
\text { EEG }\end{array}$ & $\begin{array}{l}\text { Symptom severity scores decreased. } \\
\text { three out of four patients in } \\
\text { remission (HDRS-17 } \leq 7 \text {. } \\
\text { Test-retest reliability across four } \\
\text { repeated measures over } 14 \text { months } \\
\text { met or exceeded standards for valid } \\
\text { test construction in three out of four } \\
\text { patients for cortical-evoked } \\
\text { responses studied. }\end{array}$ & N.A. \\
\hline
\end{tabular}


Table 1. Cont.

\begin{tabular}{|c|c|c|c|c|c|c|c|}
\hline Authors & Main Inclusion Criteria & No. of Patients & $\begin{array}{c}\text { Stimulation Target \& DBS } \\
\text { Design }\end{array}$ & Stimulation Parameters & $\begin{array}{c}\text { Clinical } \\
\text { Evaluation }\end{array}$ & Major Outcomes & Adverse Effects \\
\hline Smart et al. (2018) [46] & $\begin{array}{c}\text { TRD patients enrolled from } \\
\text { two separate clinical trials } \\
\text { for Deep Brain Stimulation. } \\
\text { Trial } 1 \\
\text { Both sexes aged 18-70 years; } \\
\text { Diagnosis of a Major } \\
\text { Depressive Episode or } \\
\text { Bipolar Type II-current } \\
\text { episode depressed, } \\
\text { Current episode duration of } \\
\text { at least } 1 \text { year, } \\
\text { Non-responsive (NR) } \geq 4 \\
\text { antidepressant therapies. } \\
\text { Trial } 2 \\
\text { Both sexes aged 25-70 years; } \\
\text { Current depressive episode } \\
\text { of at least } 2 \text { years duration } \\
\text { OR a history of more than } \\
\text { four lifetime } \\
\text { depressive episodes, } \\
\text { Non-responsive (NR) } \geq 4 \\
\text { antidepressant therapies. }\end{array}$ & 14 & $\begin{array}{c}\text { Target SCC } \\
\text { Study Design } \\
\text { Double-blinded. } \\
\text { Intraoperative behavioral testing: } \\
\text { Frequency: } 130 \mathrm{~Hz} \\
\text { Pulse width: } 90 \mu \mathrm{Hs} \\
\text { Current: } 6 \mathrm{~mA} \\
\text { Eight patients continued SCC } \\
\text { local field potential. }\end{array}$ & $\begin{array}{c}\frac{\text { Implantation }}{\text { Bilateral }} \\
\frac{\text { Stimulation }}{\text { Monopolar }} \\
\frac{\text { Frequency }}{130 \mathrm{~Hz}} \\
\frac{\text { Amplitude }}{\text { for }} \\
\text { 6-8 } \mathrm{mA} \text { for St. Jude Medical } \\
\text { devices, 3.5-5 V for Medtronic } \\
\text { devices } \\
\frac{\text { Pulse Width }}{90 \mu \mathrm{s}}\end{array}$ & $\begin{array}{l}\text { HDRS-17, } \\
\text { MRI, } \\
\text { LFP. }\end{array}$ & $\begin{array}{l}11 \text { of } 14 \text { patients met the criteria for } \\
\text { DBS antidepressant response by } \\
6 \text { months. } \\
\text { Of the three } 6 \text { month } \\
\text { non-responders, one responded } \\
\text { after the } 6 \text { month study endpoint } \\
\text { but without a contact change } \\
\text { (Patient 2), one responded after a } \\
\text { contact switch in the left } \\
\text { hemisphere (Patient } 7) \text {, and one } \\
\text { remained a non-responder } \\
\text { (Patient } 6 \text { ). } \\
\text { Mean baseline HDRS- } 17 \text { of } 23.8 \text { and } \\
\text { SD of } 2.8 ; \text { HDRS-17 of } 9.6 \text { and SD of } \\
4.5 \text { at month } 6 ; 19.9 \text { weeks for stable } \\
\text { response with SD of } 20 \text { weeks. } \\
\text { Precision on the left may be more } \\
\text { important than precision on the } \\
\text { right, which is supported by } \\
\text { theta decreases. }\end{array}$ & N.A. \\
\hline Choi et al. (2018) [47] & $\begin{array}{c}\text { Both sexes aged } 18-70 \text { years; } \\
\text { current MDE } \geq 12 \text { months; } \\
\text { NR } \geq 4 \\
\text { antidepressant therapies; } \\
\text { HRSD-17 score } \geq 20 ; \\
\text { GAF score } \leq 50 \text {. }\end{array}$ & 15 & $\begin{array}{c}\text { Target SCC } \\
\text { Study Design } \\
\begin{array}{c}\text { Patients went through SCC-DBS, } \\
\text { followed by MRI scans. }\end{array}\end{array}$ & $\begin{array}{l}\frac{\text { Implantation }}{\text { Bilateral }} \\
\frac{\text { Stimulation }}{\text { Monopolar }} \\
\frac{\text { Frequency }}{130 \mathrm{~Hz}} \\
\frac{\text { Amplitude }}{6 \mathrm{~mA}} \\
\frac{\text { Pulse Width }}{90 \mu \mathrm{s}}\end{array}$ & $\begin{array}{l}\text { HDRS-17, } \\
\text { MRI, DWI, Volume } \\
\text { of Tissue Activated }\end{array}$ & $\begin{array}{l}\text { Significant differences in the } \\
\text { pathway activation changes over } \\
\text { time between remitters and } \\
\text { non-remitters. } \\
\text { Non-remitters had significantly } \\
\text { larger net changes in their pathway } \\
\text { activation connection in both the } \\
\text { near and long term relative to the } \\
\text { initial plan. }\end{array}$ & N.A. \\
\hline Conen et al. (2018) [48] & $\begin{array}{c}\text { TRD (unipolar); } \\
\text { NR } \geq 4 \\
\text { antidepressant therapies; } \\
\text { MADRS Score } \geq 22 \text {. }\end{array}$ & 7 & $\begin{array}{c}\text { Targets SCC followed by Ventral } \\
\text { Anterior Capsule, nucleus } \\
\text { accumbens (separately, unless } \\
\text { patient in remission, and later } \\
\text { combined, for } \\
\text { non-responding patients). } \\
\text { Study Design DBS was applied } \\
\text { sequentially for } 3 \text { months per } \\
\text { region, for a total period ranging } \\
\text { from } 16-45 \text { months. }\end{array}$ & N.A. & $\begin{array}{l}\text { MADRS, } \\
\text { HAM-D 17, } \\
\text { GAF. }\end{array}$ & $\begin{array}{l}\text { Remitters had higher regional } \\
\text { cerebral blood flow in the baseline } \\
\text { prefrontal cortex and subsequent } \\
\text { tests when compared to } \\
\text { non-remitters and non-responders. } \\
\text { Chronic DBS increased prefrontal } \\
\text { cortex regional cerebral blood flow. } \\
\text { Remitted patients had higher } \\
\text { prefrontal cerebral blood flow } \\
\text { at baseline. }\end{array}$ & N.A. \\
\hline
\end{tabular}


Table 1. Cont

\begin{tabular}{|c|c|c|c|c|c|c|c|}
\hline Authors & Main Inclusion Criteria & No. of Patients & $\begin{array}{c}\text { Stimulation Target \& DBS } \\
\text { Design }\end{array}$ & Stimulation Parameters & $\begin{array}{c}\text { Clinical } \\
\text { Evaluation }\end{array}$ & Major Outcomes & Adverse Effects \\
\hline Holtzheimer et al. (2017) [15] & $\begin{array}{c}\text { Both sexes aged 21-70 years; } \\
\text { Unipolar, } \\
\text { non-psychotic MDD } \\
\text { First MDE onset before } \\
45 \text { years old with current } \\
\text { MDE } \geq 12 \text { months; } \\
\text { NR } \geq 4 \\
\text { antidepressant therapies, } \\
\text { MADRS Score }>22 ; G \text { GAF }< \\
50 ; \text { MMSE }>24 ; \\
\text { No changes in current } \\
\text { antidepressant treatments } \geq \\
4 \text { wks prior to study. }\end{array}$ & $\begin{array}{l}60 \text { (DBS) } \\
30 \text { (Sham) }\end{array}$ & $\begin{array}{c}\text { Target SCC } \\
\text { Study Design } \\
\text { DBS or sham stimulation } 2 \text { weeks } \\
\text { after implantation for } 6 \text { months in } \\
\text { randomized and } \\
\text { double-blind manner. } \\
\text { Two groups: DBS or sham } \\
\text { then both groups received } \\
\text { open-label stimulation for } 6 \\
\text { months or } 2 \text { years. }\end{array}$ & $\begin{array}{l}\frac{\text { Implantation }}{\text { Bilateral }} \\
\frac{\text { Stimulation }}{\text { Monopolar }} \\
\frac{\text { Frequency }}{130 \mathrm{~Hz}} \\
\frac{\text { Amplitude }}{4-8 \mathrm{~mA}} \\
\frac{\text { Pulse Width }}{91 \mu \mathrm{s}}\end{array}$ & $\begin{array}{l}\text { MADRS, GAF } \\
\text { HRSD-17, 30-item } \\
\text { Inventory of } \\
\text { Depressive } \\
\text { Symptomatology, } \\
\text { QIDS-SR, WSAS, } \\
\text { PGI, } \\
\text { CGI, QOL, } \\
\text { HAM-A. }\end{array}$ & $\begin{array}{l}\text { Insignificant difference in response } \\
\text { * between sham and DBS at the end } \\
\text { of the } 6 \text {-month double-blind phase. } \\
38 \text { patients responded }{ }^{*} \text { and } 20 \\
\text { remitted } \square \text { after } 6 \text { month DBS. } \\
\text { In } 2 \text { years of open-label active DBS, } \\
48 \% \text { achieved antidepressant } \\
\text { response and } 25 \% \text { achieved } \\
\text { remission - clinically meaningful } \\
\text { long-term outcomes. }\end{array}$ & $\begin{array}{l}\text { Severe } \\
\text { Eight of } 40 \text { events reported } \\
\text { related to device or surgery: } \\
\text { six infections (in five } \\
\text { patients), one skin erosion } \\
\text { over the extension wires, } \\
\text { and one postoperative } \\
\text { seizure. }\end{array}$ \\
\hline McInerney et al. (2017) [14] & $\begin{array}{c}\text { Current MDE } \geq 12 \text { months; } \\
\text { HRSD-17 Score } \geq 20 ; \\
\text { NR } \geq 4 \\
\text { antidepressant therapies. }\end{array}$ & 20 & $\begin{array}{c}\text { Target SCG } \\
\text { Study Design } \\
\text { DBS for 12 months open-label }\end{array}$ & $\begin{array}{l}\frac{\text { Implantation }}{\text { Bilateral }} \\
\frac{\text { Stimulation }}{\text { Monopolar }} \\
\frac{\text { Frequency }}{130 \mathrm{~Hz}} \\
\frac{\text { Amplitude }}{3.5-5 \mathrm{~V}} \\
\frac{\text { Pulse Width }}{90 \mu \mathrm{s}}\end{array}$ & $\begin{array}{c}\text { Wisconsin Card } \\
\text { Sorting Task } \\
\text { (WCST), Hopkins } \\
\text { Verbal Learning } \\
\text { Test, Controlled } \\
\text { Oral Word } \\
\text { Association Test } \\
\text { (COWA), } \\
\text { Finger Tap Test, } \\
\text { Stroop Test, } \\
\text { HRSD-17. }\end{array}$ & $\begin{array}{l}\text { Significant reduction in HRSD-17 } \\
\text { from baseline to experimental } \\
\text { follow-up. } \\
\text { Baseline scores differed } \\
\text { significantly between responders } \\
\text { and non-responders. } \\
11 \text { patients responded } * * \text { and nine } \\
\text { were non-responders. } \\
\text { WCST Test results indicated that } \\
\text { the total errors were predictive of } \\
\text { responsiveness to DBS. } \\
\text { No significant deterioration in } \\
\text { cognition and psychomotor speed. } \\
\text { Improvements in verbal memory } \\
\text { and verbal fluency. }\end{array}$ & N.A. \\
\hline Riva-Posse et al. (2018) [49] & $\begin{array}{c}\text { Both sexes aged } 18-70 \text { years; } \\
\text { current MDE } \geq 12 \text { months; } \\
\text { NR } \geq 4 \\
\text { antidepressant therapies; } \\
\text { HRSD-17 score } \geq 20 \text {; } \\
\text { GAF score } \leq 50 \text {. }\end{array}$ & 11 & $\begin{array}{c}\text { Target SCC } \\
\text { Study Design } \\
\text { DBS from } 4 \text { weeks after surgery } \\
\text { and lasted for } 6 \text { months, } \\
\text { open-label. } \\
\text { Stimulation contacts were } \\
\text { changed in non-responders and } \\
\text { they were stimulated for } \\
6 \text { more months. }\end{array}$ & $\begin{array}{l}\frac{\text { Implantation }}{\text { Bilateral }} \\
\frac{\text { Stimulation }}{\text { Monopolar }} \\
\frac{\text { Frequency }}{130 \mathrm{~Hz}} \\
\frac{\text { Amplitude }}{6-8 \mathrm{~mA}} \\
\frac{\text { Pulse Width }}{91 \mu \mathrm{s}}\end{array}$ & HRSD-17 & $\begin{array}{l}\text { Eight out of } 11 \text { responded } * * \text { and six } \\
\text { remitted } \square \square \text { after } 6 \text { month DBS. } \\
\text { Nine responded }{ }^{* *} \text { and six remitted } \\
\square \square \text { after } 12 \text { month DBS. } \\
\text { Two did not respond throughout } \\
\text { the study. } \\
\text { Tractography-based surgery } \\
\text { reduced variability in the effects of } \\
\text { stimulation on patient-specific } \\
\text { brain circuitry. }\end{array}$ & N.A. \\
\hline Tsolaki et al. (2017) [50] & TRD & 2 & Target SCC & $\begin{array}{c}\text { Implantation Bilateral } \\
\text { Stimulation Monopolar } \\
\frac{\text { Frequency }}{130 \mathrm{~Hz}} \\
\frac{\text { Amplitude }}{8 \mathrm{~mA}} \\
\frac{\text { Pulse Width }}{91 \mu \mathrm{s}}\end{array}$ & $\begin{array}{l}\text { MRI, DTI, CT, FSL } \\
\text { Probabilistic } \\
\text { tractography, } \\
\text { Volume of Tissue } \\
\text { Activated, } \\
\text { MADRS. }\end{array}$ & $\begin{array}{l}\text { One patient was a responder ( } 81 \% \\
\text { change in MADRS score). } \\
\text { Responder's contacts were closer to } \\
\text { the Tractography-guided optimized } \\
\text { target (TOT), unlike the } \\
\text { non-responder. }\end{array}$ & N.A. \\
\hline
\end{tabular}


Table 1. Cont

\begin{tabular}{|c|c|c|c|c|c|c|c|}
\hline Authors & Main Inclusion Criteria & No. of Patients & $\begin{array}{c}\text { Stimulation Target \& DBS } \\
\text { Design }\end{array}$ & Stimulation Parameters & $\begin{array}{c}\text { Clinical } \\
\text { Evaluation }\end{array}$ & Major Outcomes & Adverse Effects \\
\hline Accolla et al. (2016) [51] & $\begin{array}{c}\text { MDD; } \\
\text { NR to treatments; } \\
\text { Currently in a depressive } \\
\text { episode as in DSM-IV Axis I } \\
\text { disorders; } \\
\text { HAMD-24 score of } \geq 20 \text {. }\end{array}$ & 5 & $\begin{array}{c}\text { Target BA25 } \\
\text { Study Design } \\
\text { Double-blind. } \\
\text { Each homologous electrode pair } \\
\text { was activated separately on } \\
5 \text { consecutive days, } \\
\text { then antidepressant effects was } \\
\text { assessed } 24 \text { h later. } \\
\text { Open-label DBS for up to } \\
24 \text { months. } \\
\text { Pre- and post-DBS MRI images } \\
\text { were taken. }\end{array}$ & $\begin{array}{l}\frac{\text { Implantation }}{\text { N.A. }} \\
\frac{\text { Stimulation }}{\text { Monopolar }} \\
\frac{\text { Frequency }}{130 \mathrm{~Hz}} \\
\frac{\text { Amplitude }}{5 \mathrm{~V}} \\
\frac{\text { Pulse Width }}{90 \mu \mathrm{s}}\end{array}$ & $\begin{array}{l}\text { HAMD-24, } \\
\text { BDI. }\end{array}$ & $\begin{array}{l}\text { Four out of five patients did not } \\
\text { show sustained response ** to DBS } \\
\text { (also } \geq 50 \% \text { reduction in DBI). } \\
\text { One patient responded } * * \text { to DSS of } \\
\text { the bilateral posterior gyrus rectus } \\
\text { instead of the intended target } \\
\text { (BA25). }\end{array}$ & N.A. \\
\hline Richieri et al. (2016) [52] & $\begin{array}{l}\text { Diagnosed MDD; } \\
\text { Severe cognitive defects and } \\
\text { relapsed after ECT. }\end{array}$ & 1 & $\begin{array}{c}\text { Target BA25 } \\
\text { Study Design } \\
\text { DBS at Day } 5 \text { after } \\
\text { electrode implantation. }\end{array}$ & $\begin{array}{l}\frac{\text { Implantation }}{\text { Bilateral }} \\
\frac{\text { Stimulation }}{\text { Bipolar }} \\
\frac{\text { Frequency }}{130 \mathrm{~Hz}} \\
\frac{\text { Amplitude }}{4.2 \mathrm{~V}} \\
\frac{\text { Pulse Width }}{90 \mu \mathrm{s}}\end{array}$ & QIDS SR-16 & $\begin{array}{l}\text { Remitted (QIDS SR-16 3/48) at } \\
1 \text { month after DBS and maintained } \\
\text { at the end of DBS. }\end{array}$ & Seizure \\
\hline Hilimire et al. (2015) [53] & $\begin{array}{l}\text { Both sexes aged } 18-70 \text { years; } \\
\text { Current MDE } \geq 12 \text { months, } \\
\text { Non-responsive (NR) } \geq 4 \\
\text { antidepressant therapies, } \\
\text { HRSD-17 score } \geq 20 \text {, } \\
\text { GAF score } \leq 50 \text {. }\end{array}$ & 7 & $\begin{array}{c}\text { Target SCC } \\
\text { Study Design } \\
\text { DBS for } 6 \text { months, open-label. } \\
\text { Behavioral testing and } \\
\text { electrophysiological recording (i) } \\
\text { before electrode implantation, (ii) } \\
\text { after } 1 \text { month DBS and (iii) after } \\
6 \text { month DBS. }\end{array}$ & $\begin{array}{l}\frac{\text { Implantation }}{\text { Bilateral }} \\
\frac{\text { Stimulation }}{\text { Monopolar }} \\
\frac{\text { Frequency }}{130 \mathrm{~Hz}} \\
\frac{\text { Amplitude }}{4-8 \mathrm{~mA}} \\
\frac{\text { Pulse Width }}{91 \mu \mathrm{s}}\end{array}$ & $\begin{array}{l}\text { HDRS-17, } \\
\text { Emotional } \\
\text { self-referential task, } \\
\text { EEG recording. }\end{array}$ & $\begin{array}{l}\text { Reduced proportion of negative } \\
\text { self-descriptive words compared to } \\
\text { baseline after } 1 \text { month and } \\
6 \text { month DBS. } \\
\text { Significant reduction in P1 } \\
\text { amplitude compared to baseline } \\
\text { (for negative word self-description) } \\
\text { after } 1 \text { month and } 6 \text { month DBS, } \\
\text { and P3 amplitudes at } 6 \text { month } \\
\text { DBS only } \\
\text { Reduced depression severity. }\end{array}$ & N.A. \\
\hline $\begin{array}{l}\text { Martin-Blanco et al. } \\
\text { (2015) [54] }\end{array}$ & $\begin{array}{c}\text { Both sexes aged } 18-70 \text { years; } \\
\text { current MDE } \geq 12 \text { months; } \\
\text { NR } \geq 4 \\
\text { antidepressant therapies; } \\
\text { HRSD-17 score } \geq 20 ; \\
\text { GAF score } \leq 50 \text {. }\end{array}$ & 7 & $\begin{array}{c}\text { Target SCG } \\
\text { Study Design } \\
\text { Chronic DBS for } 9 \text { months on } \\
\text { average for clinical stabilization. } \\
\text { A PET scan was acquired } \\
\text { (i) during active stimulation and } \\
\text { (ii) after } 48 \text { hof } \\
\text { inactive stimulation. }\end{array}$ & $\begin{array}{l}\frac{\text { Implantation }}{\text { Bilateral }} \\
\text { Stimulation } \\
\frac{\text { Monopolar }}{\text { Frequency }} \\
\frac{135 \mathrm{~Hz}}{\text { Amplitude }} \\
\frac{3.5-5 \mathrm{~V}}{\text { Pulse Width }} \\
\frac{120-210 \mu \mathrm{ss}}{}\end{array}$ & HAMD-17, PET & $\begin{array}{l}\text { Decreased metabolism in BA24, } \\
\text { BA6, caudate putamen after } \\
48 \mathrm{~h} \text { DBS. } \\
\text { This study suggests metabolic } \\
\text { changes spread after longer periods } \\
\text { of no stimulation. } \\
\text { No clinical changes were detected } \\
\text { according to HAMD-17. }\end{array}$ & N.A. \\
\hline
\end{tabular}


Table 1. Cont.

\begin{tabular}{|c|c|c|c|c|c|c|c|}
\hline Authors & Main Inclusion Criteria & No. of Patients & $\begin{array}{c}\text { Stimulation Target \& DBS } \\
\text { Design }\end{array}$ & Stimulation Parameters & $\begin{array}{c}\text { Clinical } \\
\text { Evaluation }\end{array}$ & Major Outcomes & Adverse Effects \\
\hline Puigdemont et al. (2015) [55] & $\begin{array}{c}\text { Severe TRD; } \\
\text { Both sexes aged } 18-70 \text { years; } \\
\text { current MDE } \geq 12 \text { months; } \\
\text { NR } \geq 4 \text { antidepressant } \\
\text { therapies; } \\
\text { HRSD-17 score } \geq 20 ; \\
\text { GAF score } \leq 50 \text {. }\end{array}$ & 5 & $\begin{array}{c}\text { Target SCG } \\
\text { Study Design } \\
\text { Randomized, Double-blind. } \\
\text { After stable clinical remission to } \\
\text { DBS, patients were allocated to } \\
\text { two groups, one with } \\
\text { (i) } 3 \text { month DBS-ON, then } \\
\text { (ii) } 3 \text { month sham stimulation } \\
\text { (ON-OFF arm) or OFF-ON arm } \\
\text { and the other, vice-versa. }\end{array}$ & $\begin{array}{l}\frac{\text { Implantation }}{\text { Bilateral }} \\
\frac{\text { Stimulation }}{\text { Monopolar }} \\
\text { Frequency } \\
130-135 \mathrm{~Hz} \\
\frac{\text { Amplitude }}{3.5-5 \mathrm{~V}} \\
\frac{\text { Pulse Width }}{120-240 \mu \mathrm{ss}}\end{array}$ & $\begin{array}{l}\text { Volume of Tissue } \\
\text { Activated, } \\
\text { HRSD-17 }\end{array}$ & $\begin{array}{l}\text { Active stimulation: four of five } \\
\text { patients were remitted patients. } \\
\text { Sham stimulation: Only two } \\
\text { patients remained in remission, } \\
\text { another two relapsed, and one } \\
\text { showed a progressive worsening } \\
\text { without reaching relapse criteria. }\end{array}$ & N.A. \\
\hline Serra-Blasco et al. (2015) [56] & $\begin{array}{c}\text { Treatment-Resistant Depression } \\
\text { (TRD) Group } \\
\text { Resistant to } \\
\text { pharmacological treatment; } \\
\text { min. stage IV of } \\
\text { Thase-Rush scale; } \\
\text { HDRS score } \geq 18 . \\
\text { First Episode MDD } \\
\text { (FE MDD) Group } \\
\text { HDRS score } \geq 14 ; \\
\text { Newly diagnosed MDD. }\end{array}$ & 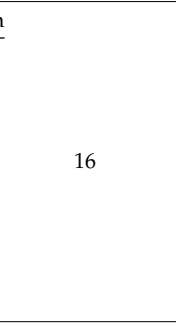 & $\begin{array}{c}\text { Target SCG } \\
\text { Study Design } \\
\text { DBS began at } 48 \text { h postoperative } \\
\text { and ended when each patient had } \\
\text { stabilized response for at least } \\
\text { three consecutive visits, tests } \\
\text { conducted before surgery, and } \\
12 \text { months after DBS treatment. }\end{array}$ & $\begin{array}{l}\frac{\text { Implantation }}{\text { Bilateral }} \\
\frac{\text { Stimulation }}{\text { Monopolar }} \\
\frac{\text { Frequency }}{135 \mathrm{~Hz}} \\
\frac{\text { Amplitude }}{3.5-5 \mathrm{~V}} \\
\frac{\text { Pulse Width }}{120-210 \mu \mathrm{s}}\end{array}$ & $\begin{array}{c}\text { Rey Auditory } \\
\text { Verbal Learning } \\
\text { Test, Trail Making } \\
\text { Tests-A and -B, } \\
\text { Wechsler Adult } \\
\text { Intelligence Scale } \\
\text { III, Tower of } \\
\text { London Test, } \\
\text { HDRS-17. }\end{array}$ & $\begin{array}{l}\text { FE MDD and TRD saw significant } \\
\text { improvements over time } \\
\text { in memory. } \\
\text { No significant difference was } \\
\text { observed in both groups on } \\
\text { executive functioning, language, } \\
\text { and processing speed. } \\
\text { DBS was well tolerated and had no } \\
\text { adverse effect on } \\
\text { neuropsychological and } \\
\text { cognitive function. }\end{array}$ & N.A. \\
\hline Choi et al. (2015) [57] & $\begin{array}{c}\text { Both sexes aged } 18-70 \text { years; } \\
\text { current MDE } \geq 12 \text { months; } \\
\text { NR } \geq 4 \\
\text { antidepressant therapies; } \\
\text { HDRS-17 score } \geq 20 ; \\
\text { GAF score } \leq 50 \text {. }\end{array}$ & 9 & $\begin{array}{c}\text { Target SCC } \\
\text { Intraoperative Sessions: } 6 \text { min } \\
\text { session of } 3 \text { min stimulation ON, } \\
\text { and } 3 \text { min stimulation OFF. } \\
\text { Number of sessions: } \\
12 \text { trials (one at each of the } \\
\text { eight available contacts; four per } \\
\text { hemisphere, plus four } \\
\text { sham trials). } \\
\text { Study Design } \\
\text { Sham-controlled, } \\
\text { Double-blind trials (one case } \\
\text { w/single blind trial). }\end{array}$ & $\begin{array}{c}\begin{array}{c}\text { Acute } \\
\text { Implantation }\end{array} \\
\text { Bilateral } \\
\frac{\text { Stimulation }}{\text { Monopolar }} \\
\frac{\text { Frequency }}{130 \mathrm{~Hz}} \\
\frac{\text { Amplitude }}{6 \mathrm{~mA}} \\
\frac{\text { Pulse Width }}{90 \mu \mathrm{s}}\end{array}$ & $\begin{array}{c}\text { MRI with FSL } \\
\text { analysis, } \\
\text { Volume of } \\
\text { Tissue Activated. }\end{array}$ & $\begin{array}{l}\text { Behavioral switch was apparent to } \\
\text { patients within the first minute of } \\
\text { the initiation of stimulation and } \\
\text { effects were sustained while } \\
\text { stimulation remained on. } \\
\text { Three common white matter } \\
\text { bundles were affected by } \\
\text { stimulation: (i) the uncinate } \\
\text { fasciculus, (ii) the forceps minor, } \\
\text { and (iii) the left cingulum } \\
\text { bundle.Seven of nine patients with } \\
\text { left hemispheric contact had a } \\
\text { response to treatment at } 6 \text { months. }\end{array}$ & \\
\hline Sun et al. (2015) [58] & $\begin{array}{c}\text { NR } \geq 4 \text { antidepressant } \\
\text { therapies, } \\
\text { Mean HRSD-17 score of } \\
25 \text { (3). }\end{array}$ & 20 & $\begin{array}{c}\text { Target SCC } \\
\text { Session: 100 min, } \mathrm{w} / 15 \text { min break } \\
\text { EEG recording sessions/day } \\
\text { Session 1: DBS On } \\
\text { Session 2: DBS Random (On/Off) } \\
\text { Session 3: DBS (Off) }\end{array}$ & $\begin{array}{l}\frac{\text { Implantation }}{\text { Bilateral }} \\
\text { Stimulation } \\
\text { Monopolar } \\
\text { Frequency } \\
130 \mathrm{~Hz} \\
\text { Amplitude } \\
2-7.25 \mathrm{~mA} \mathrm{OR} \\
2-6 \mathrm{~V} \\
\frac{\text { Pulse Width }}{90 \mu \mathrm{s}}\end{array}$ & $\begin{array}{l}\text { EEG, } \\
\text { HDRS-17. }\end{array}$ & $\begin{array}{l}\text { Suppression of gamma oscillations } \\
\text { by DBS during working memory } \\
\text { performance and the treatment } \\
\text { efficacy of DBS for TRD may be } \\
\text { associated with the improved } \\
\text { GABAergic neurotransmission, } \\
\text { previously shown to be deficient in } \\
\text { MDD. The present study also } \\
\text { suggests that modifying treatment } \\
\text { parameters to achieve suppression } \\
\text { of gamma oscillations and } \\
\text { increased theta-gamma coupling } \\
\text { may lead to optimized DBS efficacy } \\
\text { for TRD. }\end{array}$ & N.A. \\
\hline
\end{tabular}


Table 1. Cont

\begin{tabular}{|c|c|c|c|c|c|c|c|}
\hline Authors & Main Inclusion Criteria & No. of Patients & $\begin{array}{c}\text { Stimulation Target \& DBS } \\
\text { Design }\end{array}$ & Stimulation Parameters & $\begin{array}{c}\text { Clinical } \\
\text { Evaluation }\end{array}$ & Major Outcomes & Adverse Effects \\
\hline $\begin{array}{l}\text { Perez-Caballero et al. } \\
\text { (2014) [59] }\end{array}$ & $\begin{array}{l}18-70 \text { years old with MDE; } \\
\text { Resistant to } \\
\text { pharmacological treatment } \\
\text { and at most, a partial } \\
\text { response to ECT; } \\
\text { HAMD-17 Score } \geq 18 \text {. }\end{array}$ & 8 & $\begin{array}{l}\text { Target SCG } \\
\text { Study Design All patients } \\
\text { received chronic DBS within } 48 \mathrm{~h} \\
\text { after implantation. } \\
\text { Four patients took NSAIDs for up } \\
\text { to } 30 \text { days postoperative, four } \\
\text { patients did not. }\end{array}$ & $\begin{array}{l}\text { Implantation } \\
\text { Quadrupolar } \\
\frac{\text { Stimulation }}{135 \mathrm{~Hz}} \\
\frac{\text { Amplitude }}{3.5-5 \mathrm{~V}} \\
\frac{\text { Pulse Width }}{120-210 \mu \mathrm{s}}\end{array}$ & HDRS-17 & $\begin{array}{l}\text { At week } 1 \text { after surgery, all patients } \\
\text { without NSAID prescription } \\
\text { responded }{ }^{* *} \text { and two remitted } \square \square \text {; } \\
\text { three patients with NSAID } \\
\text { responded }{ }^{* *} \text {, and two remitted } \\
\text { At week } 4 \text { after surgery, three } \\
\text { patients without NSSID remitted } \\
\text { qu; no patients with NSAID } \\
\text { responded }{ }^{* *}\end{array}$ & N.A. \\
\hline Merkl et al. (2013) [60] & $\begin{array}{c}\text { MDD; } \\
\text { NR to treatments; } \\
\text { Currently in a depressive } \\
\text { episode as in DSM-IV Axis } \\
\text { I disorder; } \\
\text { HAMD-24 score of } \geq 20 ; \\
\text { HDRS-24 score } \geq 24 \text {. }\end{array}$ & 6 & $\begin{array}{l}\text { Target SCG } \\
\text { Study Design DBS on } 11-19 \text { days } \\
\text { after electrode implantation, } 24 \mathrm{~h} \\
\text { acute stimulation followed by } \\
\text { sham stimulation for each of the } \\
\text { three electrode pairs. } \\
\text { Up to } 6 \text { months of } \\
\text { chronic stimulation. }\end{array}$ & $\begin{array}{l}\frac{\text { Implantation }}{\text { Bilateral }} \\
\frac{\text { Stimulation }}{\text { Monopolar }} \\
\frac{\text { Frequency }}{130 \mathrm{~Hz}} \\
\frac{\text { Amplitude }}{2.5-10 \mathrm{~V}} \\
\frac{\text { Pulse Width }}{90 \mu \mathrm{s}}\end{array}$ & $\begin{array}{c}\text { HAMD-24, } \\
\text { MADRS } \\
\text { BDI, } \\
\text { TMT-A, TMT-B, } \\
\text { CVLT, TAP, Boston } \\
\text { Naming Test, } \\
\text { Stroop Test, Word } \\
\text { Fluency Test. }\end{array}$ & $\begin{array}{l}\text { Non-significant reduction in } \\
\text { HAMD-24, BDI, and MADRS } \\
\text { scores for acute DBS and } \\
\text { sham stimulation. } \\
\text { 0/4 contact pair locations showed } \\
\text { significant BDI and } \\
\text { MADRS improvements. } \\
\text { Contact pair } 3 / 7 \text { for Patient } 4 \text { saw a } \\
77 \% \text { reduction in HAMDD-24 score } \\
\text { and } 62 \% \text { reduction of MADRS score. } \\
\text { Reduced HDRS-24, BDI, and } \\
\text { MADRS scores at the end of } \\
\text { chronic stimulation. } \\
\text { Two out of six remissions }{ }^{\circ} \text { at the } \\
\text { end of chronic stimulation. }\end{array}$ & $\begin{array}{c}\text { Mild } \\
\text { Headache; } \\
\text { Pain; } \\
\text { Scalp tingling; } \\
\text { Dizziness; } \\
\text { Sore throat; } \\
\text { Hardware-related; } \\
\frac{\text { Severe }}{\text { NIL }}\end{array}$ \\
\hline Ramasubbu et al. (2013) [61] & $\begin{array}{c}\text { Aged between } 20-60 \text { years; } \\
\text { Diagnosed MDD; } \\
\text { HAMD-17 score } \geq 20 ; \\
\text { NR } \geq 4 \\
\text { antidepressant therapies. } \\
\text { (Enrolled patients were } \\
\text { among the most } \\
\text { treatment resistant). }\end{array}$ & 4 & $\begin{array}{c}\text { Target SCC } \\
\text { Study Design } \\
\text { Double-blind DBS optimization. } \\
\text { Open-label continuous DBS for } 6 \\
\text { months after optimization period. } \\
\text { Varied parameters for each } \\
\text { patient during optimization. }\end{array}$ & $\begin{array}{l}\text { Implantation } \\
\text { Bilateral Stimulation } \\
\text { Monopolar } \\
\text { Frequency } \\
0 / 5 / 20 / 50 / 130 / 185 \mathrm{~Hz} \\
\text { Amplitude } \\
0-10.5 \mathrm{~V} \\
\frac{\text { Pulse Width }}{0 / 90 / 150 /} \\
270 / 450 \text { us }\end{array}$ & $\begin{array}{l}\text { HAMD-17, } \\
\text { MADRS, } \\
\text { HAM-A, } \\
\text { CGI. }\end{array}$ & $\begin{array}{l}\text { Postoperative optimization: All } \\
\text { four patients showed maximal } \\
\text { response at longer pulse widths; } \\
\text { three patients experienced a 50\% } \\
\text { reduction in HAMDD-17 score. } \\
\text { Longer pulse widths were } \\
\text { correlated to short-term } \\
\text { improvement. Longer pulse width } \\
\text { also induced insomnia, confusion, } \\
\text { and drowsiness; improved by } \\
\text { turning off stimulation. } \\
\text { Chronic stimulation: two patients } \\
\text { responded *ै at the end of } \\
\text { open-label DBS, with longer } \\
\text { pulse width. } \\
\text { Electrode targets suggested to be } \\
\text { individualized, as opposed to } \\
\text { standard as in movement disorders, } \\
\text { owing to the complexity of cortical } \\
\text { gyral anatomy }\end{array}$ & $\begin{array}{l}\text { Mild } \\
\text { Anxiety; } \\
\text { Drowsiness; } \\
\text { Confusion; } \\
\text { Insomnia. }\end{array}$ \\
\hline
\end{tabular}


Table 1. Cont

\begin{tabular}{|c|c|c|c|c|c|c|c|}
\hline Authors & Main Inclusion Criteria & No. of Patients & $\begin{array}{c}\text { Stimulation Target \& DBS } \\
\text { Design }\end{array}$ & Stimulation Parameters & $\begin{array}{c}\text { Clinical } \\
\text { Evaluation }\end{array}$ & Major Outcomes & Adverse Effects \\
\hline Torres et al. (2013) [62] & $\begin{array}{l}\text { Type I bipolar depression; } \\
\text { Poor response to ECT and } \\
\text { pharmacotherapy. }\end{array}$ & 1 & $\begin{array}{c}\text { Target SCC } \\
\text { Study Design } \\
\text { DBS from 15 days after } \\
\text { implantation and follow-up for } \\
9 \text { months. }\end{array}$ & $\begin{array}{l}\frac{\text { Implantation }}{\text { N.A. }} \\
\frac{\text { Stimulation }}{\text { Monopolar }} \\
\frac{\text { Frequency }}{130 \mathrm{~Hz}} \\
\frac{\text { Amplitude }}{6 \mathrm{~mA}} \\
\frac{\text { Pulse Width }}{91 \mu \mathrm{s}}\end{array}$ & $\begin{array}{l}\text { HDRS-17, } \\
\text { BDI, } \\
\text { MADRS, } \\
\text { GAF, } \\
\text { Young Mania } \\
\text { Scale. }\end{array}$ & $\begin{array}{l}\text { Scores improved across tests. } \\
\text { Psychotic symptoms disappeared. } \\
\text { Manic episodes reduced. }\end{array}$ & N.A. \\
\hline Broadway et al. (2012) [63] & $\begin{array}{c}\text { Both sexes aged } 18-70 \text { years; } \\
\text { current MDE } \geq 12 \text { month; } \\
\text { NR } \geq 4 \\
\text { antidepressant therapies; } \\
\text { HRSD-17 score } \geq 20 ; \\
\text { GAF score } \leq 50 \text {. }\end{array}$ & 12 & $\begin{array}{c}\text { Target SCC } \\
\text { Study Design } \\
\text { DBS for up to } 24 \text { weeks. }\end{array}$ & $\begin{array}{l}\frac{\text { Implantation }}{\text { N.A. }} \\
\frac{\text { Stimulation }}{\text { Monopolar }} \\
\frac{\text { Frequency }}{130 \mathrm{~Hz}} \\
\frac{\text { Amplitude }}{6-8 \mathrm{~mA}} \\
\frac{\text { Pulse Width }}{90 \mu \mathrm{s}}\end{array}$ & $\begin{array}{l}\text { HRSD-17, } \\
\text { Frontal and } \\
\text { posterior Theta } \\
\text { cordance. }\end{array}$ & $\begin{array}{l}\text { Reduced HDRS-17 scores between } \\
\text { baseline and the end of DBS among } \\
\text { all patients. } \\
\text { Six patients had significantly } \\
\text { reduced HRSD-17 scores } * \text { at the } \\
\text { end of DBS. } \\
\text { Increased frontal theta cordance } \\
\text { between baseline and week } 4 \text { in } \\
\text { responders correlated with their } \\
\text { decreased depressive state at later } \\
\text { time points. }\end{array}$ & N.A. \\
\hline Hamani et al. (2012) [64] & $\begin{array}{l}\text { TRD; } \\
\text { NR to respond to } \\
\text { pharmacotherapy, } \\
\text { psychotherapy, transcranial } \\
\text { magnetic stimulation, ECT, } \\
\text { vagus nerve stimulation. } \\
\text { Relapsed after receiving } 6 \\
\text { month SCC-DBS. }\end{array}$ & 1 & $\begin{array}{c}\text { Target SCC } \\
\text { Study Design Administered } \\
\text { tranylcypromine before surgery. } \\
\text { DBS for } 6 \text { months. }\end{array}$ & $\begin{array}{l}\frac{\text { Implantation }}{\text { Bilateral }} \\
\frac{\text { Stimulation }}{\text { Monopolar }} \\
\frac{\text { Frequency }}{130 \mathrm{~Hz}} \\
\frac{\text { Amplitude }}{2.5 \mathrm{~V}} \\
\frac{\text { Pulse Width }}{90 \mu \mathrm{s}} \\
\end{array}$ & HAMD-17 & $\begin{array}{l}\text { Before relapse: SCC-DBS reduced } \\
\text { HAMD-17 score from } 22 \text { to } 9 \text { after } 4 \\
\text { month DBS. } \\
\text { After relapse: MAOI } \\
\text { supplementation restored the } \\
\text { therapeutic effect of DBS; } \\
\text { HAMD-17 score lowered from } 22 \text { to } \\
16 \text { (after } 2 \text { weeks), to } 8 \text { (after } 2 \\
\text { months) and to } 9 \text { (after } 4 \text { months). }\end{array}$ & N.A. \\
\hline Holtzheimer et al. (2012) [65] & $\begin{array}{c}\text { Both sexes aged } 18-70 \text { years; } \\
\text { current MDE } \geq 12 \text { months; } \\
\text { NR } \geq 4 \\
\text { antidepressant therapies; } \\
\text { HRSD-17 score } \geq 20 ; \\
\text { GAF score } \leq 50 \text {. }\end{array}$ & 17 & $\begin{array}{c}\text { Target SCC } \\
\text { Study Design } \\
\text { Intraoperative testing of electrode } \\
\text { location for } 12 \text { or } 17 \text { patients. } \\
\text { Stimulation: } \\
\text { (i) } 4 \text { weeks of sham stimulation, } \\
\text { followed by } \\
\text { (ii) } 24 \text { weeks of open label DBS } \\
\text { for } 24 \text { weeks, followed by } \\
\text { (iii) single-blind discontinuation } \\
\text { for } 1 \text { week and open label } \\
\text { stimulation for up to } 2 \text { years. }\end{array}$ & $\begin{array}{l}\frac{\text { Implantation }}{\text { Bilateral }} \\
\frac{\text { Stimulation }}{\text { Monopolar }} \\
\frac{\text { Frequency }}{130 \mathrm{~Hz}} \\
\frac{\text { Amplitude }}{4-8 \mathrm{~mA}} \\
\frac{\text { Pulse Width }}{91 \mu \mathrm{s}}\end{array}$ & $\begin{array}{l}\text { HRSD-17, } \\
\text { BDI-II, } \\
\text { GAF. }\end{array}$ & $\begin{array}{l}\text { Reduced depression and } \\
\text { increased function. } \\
11 \text { patients responded } * * \text { and seven } \\
\text { further remitted } \square \square \text { after } 2 \text { year DBS. } \\
\text { Efficacy was similar for patients } \\
\text { with MDD and those with BP. } \\
\text { A modest sham stimulation effect } \\
\text { was found, likely due to a decrease } \\
\text { in depression after the surgical } \\
\text { intervention, but prior to entering } \\
\text { the sham phase. }\end{array}$ & $\begin{array}{c}\text { Anxiety; } \\
\text { Worsened depression, } \\
\text { Nausea; } \\
\text { Headache; } \\
\text { Infection; } \\
\text { Suicide attempts. }\end{array}$ \\
\hline
\end{tabular}


Table 1. Cont.

\begin{tabular}{|c|c|c|c|c|c|c|c|}
\hline Authors & Main Inclusion Criteria & No. of Patients & $\begin{array}{c}\text { Stimulation Target \& DBS } \\
\text { Design }\end{array}$ & Stimulation Parameters & $\begin{array}{c}\text { Clinical } \\
\text { Evaluation }\end{array}$ & Major Outcomes & Adverse Effects \\
\hline Lozano et al. (2012) [66] & $\begin{array}{c}\text { Both sexes aged } 30-60 \text { years; } \\
\text { First MDE before } 35 \text { years; } \\
\text { HRSD-17 score } \geq 20 ; \\
\text { GAF }<50 \text {. }\end{array}$ & 21 & $\begin{array}{c}\text { Target SCG } \\
\frac{\text { Study Design DBS for } 12 \text { months, }}{\text { open label. }}\end{array}$ & $\begin{array}{l}\frac{\text { Implantation }}{\text { Bilateral }} \\
\text { Stimulation } \\
\text { N.A. } \\
\frac{\text { Frequency }}{110-140 \mathrm{~Hz}} \\
\frac{\text { Amplitude }}{3.5-7 \mathrm{~mA}} \\
\frac{\text { Pulse Width }}{65-182 \mu \mathrm{s}}\end{array}$ & $\begin{array}{l}\text { HRSD-17, } \\
\text { CGI-S. }\end{array}$ & $\begin{array}{l}\text { Improved global functioning and } \\
\text { less severe depression. } \\
13 \text { patients responded }{ }^{* * *} \text {, based on } \\
\text { HRSD-17 scores. }\end{array}$ & $\begin{array}{l}\text { Gastrointestinal problems; } \\
\text { Skin problem; } \\
\text { Suicide; } \\
\text { Spasms; } \\
\text { Weight gain; } \\
\text { Insomnia. }\end{array}$ \\
\hline Puigdemont et al. (2012) [67] & $\begin{array}{l}18-70 \text { years old with MDE; } \\
\text { Resistant to } \\
\text { pharmacological treatment } \\
\text { and at most, a partial } \\
\text { response to ECT; } \\
\text { HAMD-17 Score } \geq 18 .\end{array}$ & 8 & $\begin{array}{l}\text { Target SCG } \\
\text { Study Design Intraoperative } \\
\text { feedback was provided during } \\
\text { surgery for electrode placement. } \\
\text { DBS began at } 48 \mathrm{~h} \text { postoperative } \\
\text { and ended when each patient had } \\
\text { stabilized their response for at } \\
\text { least three consecutive visits. }\end{array}$ & $\begin{array}{l}\frac{\text { Implantation }}{\text { Bilateral }} \\
\text { Stimulation } \\
\frac{\text { Monopolar }}{\text { Frequency }} \\
\frac{135 \mathrm{~Hz}}{\text { Amplitude }} \\
\frac{\text { Am-5 V }}{\text { Pulse Width }} \\
90 \mu \mathrm{s}\end{array}$ & $\begin{array}{l}\text { HAMD-17, } \\
\text { MADRS, } \\
\text { CGI. }\end{array}$ & $\begin{array}{l}\text { Seven patients responded ** and } \\
\text { three remitted } \square \text { व after } \\
6 \text { month DBS. } \\
\text { Five patients responded }{ }^{* *} \text { and four } \\
\text { remitted } \square \square \text { after } 12 \text { month DBS. } \\
\text { Three out of four remitted patients } \\
\text { after } 12 \text { month DBS had remitted } \\
\text { after } 3 \text { month DBS. }\end{array}$ & $\begin{array}{l}\text { Suicide ideation; } \\
\text { Neck pain; } \\
\text { Recurrence; Depression; } \\
\text { Cephalalgia. }\end{array}$ \\
\hline Kennedy et al. (2011) [18] & $\begin{array}{l}\text { Current MDE } \geq 12 \text { months; } \\
\text { HRSD-17 score } \geq 20 ; \\
\text { NR } \geq 4 \\
\text { antidepressant therapies. }\end{array}$ & 20 & $\begin{array}{c}\text { Target SCG } \\
\text { DBS patients were monitored for } \\
\text { 3-6 years. }\end{array}$ & $\begin{array}{l}\frac{\text { Implantation }}{\text { Bilateral }} \\
\text { Stimulation } \\
\text { Monopolar } \\
\text { Frequency } \\
\text { 124.7 Hz (average) } \\
\text { Amplitude } \\
4.3 \mathrm{~V} \text { (average) } \\
\frac{\text { Pulse Width }}{70.6 \mathrm{ss}}\end{array}$ & $\begin{array}{l}\text { HAMD-17 } \\
\text { 36-item } \\
\text { Short-Form } \\
\text { Healthy Survey } \\
\text { Questionnaire. }\end{array}$ & $\begin{array}{l}64.3 \% \text { patients responded }{ }^{* *} \text { at the } \\
\text { last follow-up visit. } \\
35 \% \text { patients remitted } \text { व } \text { at the last } \\
\text { follow-up visit. } \\
\text { Scores at the last visit tended } \\
\text { towards maintenance of } \\
\text { therapeutic scores at } 3 \text { years. }\end{array}$ & $\begin{array}{l}\text { Depression; } \\
\text { Suicidal thoughts; } \\
\text { Suicide } \\
\text { (All determined to be } \\
\text { unrelated to DBS). }\end{array}$ \\
\hline Guinjoan et al. (2010) [68] & $\begin{array}{l}\text { Chronic TRD; } \\
\text { Family history of } \\
\text { affective disorders; } \\
\text { Poor response to } \\
\text { antidepressants, ECT, } \\
\text { and psychotherapy. }\end{array}$ & 1 & $\begin{array}{l}\text { Target BA25 } \\
\text { Study Design } \\
\text { Positioning was aided by } \\
\text { intraoperative feedback. } \\
\text { Bilateral DBS was conducted for } \\
12 \text { months. } \\
\text { Followed by unilateral-left, then } \\
\text { right DBS, for } 6 \text { months }\end{array}$ & $\begin{array}{l}\frac{\text { Implantation }}{\text { Bilateral }} \\
\frac{\text { Stimulation }}{\text { Monopolar }} \\
\text { Frequency } \\
\frac{130 \mathrm{~Hz}}{3.5-5 \mathrm{~V}} \\
\frac{\text { Amplitude }}{3.5-5} \\
\frac{\text { Pulse Width }}{90 \mu \mathrm{s}}\end{array}$ & $\begin{array}{l}\text { HAMD-17, } \\
\text { BDI. }\end{array}$ & $\begin{array}{l}\text { Patient's condition plateaued after } \\
6 \text { month bilateral DBS. } \\
\text { Left unilateral DBS led to rapid } \\
\text { worsening in mood. } \\
\text { Right unilateral DBS reversed the } \\
\text { symptoms and the patient made } \\
\text { significant improvements over } \\
\text { bilateral stimulation. Patient } \\
\text { remitted at } 18 \text { months. }\end{array}$ & Orthostatic hypotension. \\
\hline
\end{tabular}


Table 1. Cont.

\begin{tabular}{|c|c|c|c|c|c|c|c|}
\hline Authors & Main Inclusion Criteria & No. of Patients & $\begin{array}{c}\text { Stimulation Target \& DBS } \\
\text { Design }\end{array}$ & Stimulation Parameters & $\begin{array}{c}\text { Clinical } \\
\text { Evaluation }\end{array}$ & Major Outcomes & Adverse Effects \\
\hline $\begin{array}{l}\text { Holtzheimer and Mayberg } \\
\text { (2010) [69] }\end{array}$ & $\begin{array}{l}\text { Showed signs and } \\
\text { symptoms of MDD; } \\
\text { Had suicidal ideation in } \\
\text { current MDE; } \\
\text { Did not improve in } \\
\text { symptoms with } \\
\text { pharmacotherapy, } \\
\text { psychotherapy, and ECT } \\
\text { HAM-D score was } 25\end{array}$ & 1 & $\begin{array}{c}\text { Target SCC } \\
\text { Study Design Positioning of } \\
\text { electrodes was aided by } \\
\text { intraoperative feedback } \\
24 \text { weeks of open-label DBS and } \\
\text { chronic stimulation beyond } \\
\text { the assessment }\end{array}$ & $\begin{array}{l}\frac{\text { Implantation }}{\text { Bilateral }} \\
\frac{\text { Stimulation }}{\text { Monopolar }} \\
\frac{\text { Frequency }}{130 \mathrm{~Hz}} \\
\frac{\text { Amplitude }}{6 \mathrm{~mA}} \\
\frac{\text { Pulse Width }}{91 \mu \mathrm{s}} \\
\end{array}$ & HAMD-17 & $\begin{array}{l}\text { HAMD-17 score lowered to } 9 \text { at the } \\
\text { end of DBS follow-up. } \\
\text { Sustained antidepressant response } \\
\text { up to } 2 \text { years after surgery }\end{array}$ & N.A. \\
\hline Hamani et al. (2009) [70] & $\begin{array}{l}\text { Diagnosed MDD; } \\
\text { current MDE } \geq 12 \text { months; } \\
\text { HAMD-17 score }>20 ; \\
\text { GAF } \leq 50 ; \\
\text { NR } \geq 4 \\
\text { antidepressant therapies. }\end{array}$ & 20 & $\begin{array}{c}\text { Target SCG } \\
\text { Study Design DBS began at } 2 \\
\text { weeks after surgery and lasted for } \\
12 \text { months }\end{array}$ & $\begin{array}{l}\frac{\text { Implantation }}{\text { Bilateral }} \\
\frac{\text { Stimulation }}{\text { Monopolar }} \\
\frac{\text { Frequency }}{130 \mathrm{~Hz}} \\
\frac{\text { Amplitude }}{3-5 \mathrm{~V}} \\
\frac{\text { Pulse Width }}{90 \mu \mathrm{s}}\end{array}$ & HAMD-17 & $\begin{array}{l}11 \text { responded }{ }^{* *} \text { at the end of } 6 \\
\text { month DBS follow-up. } \\
\text { Electrodes in responders were } \\
\text { positioned ventrally relative to the } \\
\text { landmarks of the medial } \\
\text { prefrontal lobe. }\end{array}$ & N.A. \\
\hline Puigdemont et al. (2009) [71] & $\begin{array}{l}\text { Suffered from MDD, with } \\
\text { several MDE accompanied } \\
\text { by psychotic symptoms; } \\
\text { Responded poorly to } \\
\text { pharmacotherapy and ECT; } \\
\text { Relapse following SCG-DBS } \\
\text { with different features; } \\
\text { Psychotic as opposed to } \\
\text { depressive from } \\
\text { previous episodes. }\end{array}$ & 1 & $\begin{array}{c}\text { Target SCG } \\
\text { Study Design DBS for } 4 \text { months, } \\
\text { then switched off because of } \\
\text { relapse and administered ECT for } \\
3 \text { weeks } \\
\text { Resumed DBS until } 12 \text { months } \\
\text { from the beginning of DBS. }\end{array}$ & $\begin{array}{l}\frac{\text { Implantation }}{\text { Bilateral }} \\
\frac{\text { Stimulation }}{\text { Monopolar }} \\
\frac{\text { Frequency }}{135 \mathrm{~Hz}} \\
\frac{\text { Amplitude }}{3.6 \mathrm{~V}} \\
\frac{\text { Pulse Width }}{90 \mu \mathrm{s}}\end{array}$ & HAMD-17 & $\begin{array}{l}\text { Sustained response without the } \\
\text { need of ECT before relapse. } \\
\text { Maintained remission in DBS after } \\
\text { ECT until the end of follow-up. }\end{array}$ & N.A. \\
\hline
\end{tabular}


Table 1. Cont.

\begin{tabular}{|c|c|c|c|c|c|c|c|}
\hline Authors & Main Inclusion Criteria & No. of Patients & $\begin{array}{c}\text { Stimulation Target \& DBS } \\
\text { Design }\end{array}$ & Stimulation Parameters & $\begin{array}{c}\text { Clinical } \\
\text { Evaluation }\end{array}$ & Major Outcomes & Adverse Effects \\
\hline Lozano et al. (2008) [72] & $\begin{array}{l}\text { Current MDE } \geq 12 \text { months; } \\
\text { HRSD-17 score } \geq 20 ; \\
\text { NR } \geq 4 \\
\text { antidepressant therapies. }\end{array}$ & 20 & $\begin{array}{l}\text { Target SCG } \\
\text { Study Design Blinded-DBS in } \\
\text { between and after surgery, } \\
\text { monitored for up to } 1 \text { year. }\end{array}$ & $\begin{array}{l}\frac{\text { Implantation }}{\text { Bilateral }} \\
\frac{\text { Stimulation }}{\text { Monopolar }} \\
\frac{\text { Frequency }}{130 \mathrm{~Hz}} \\
\frac{\text { Amplitude }}{3.5-5 \mathrm{~V}} \\
\frac{\text { Pulse Width }}{90 \mu \mathrm{s}}\end{array}$ & $\begin{array}{l}\text { HRSD-17, Beck } \\
\text { Anxiety Inventory, } \\
\text { BDI, CGI-S, } \\
\text { PET scans, } \\
\text { Neuropsychological } \\
\text { tests. }\end{array}$ & $\begin{array}{l}\text { Mean HRSD-17 score lower than } \\
\text { baseline at all time points. } \\
12 \text { patients responded } \\
\text { remitted } \square \text { after } 6 \text { month DBS, } 7 \\
11 \text { patients responded }{ }^{* *} \text { Do DBS, } 7 \\
\text { were nearly remitted or remitted } \text { 口ם } \\
\text { after } 12 \text { month DBS. } \\
\text { Eight responses maintained from } 6 \\
\text { month to } 12 \text { month DBS. } \\
\text { PET Scans: decreases in orbital, } \\
\text { medial prefrontal cortex, and } \\
\text { insula. Increases in lateral } \\
\text { prefrontal cortex, parietal, anterior, } \\
\text { and posterior cingulate by } 6 \\
\text { months; increases in metabolic } \\
\text { activity in regions adjacent to SCG. }\end{array}$ & $\begin{array}{l}\text { Seven patients without } \\
\text { adverse effects. } \\
\text { Wound Infection; } \\
\text { Headache; } \\
\text { Pain; } \\
\text { Seizure; } \\
\text { Worsened mood; } \\
\text { Irritability. }\end{array}$ \\
\hline McNeely et al. (2008) [73] & $\begin{array}{l}\text { Current MDE } \geq 12 \text { months, } \\
\text { HRSD-17 score } \geq 20, \\
\text { Non-responsive (NR) } \geq 4 \\
\text { antidepressant therapies, }\end{array}$ & 6 & $\begin{array}{l}\text { Target BA25 } \\
\text { Continuous DBS for } 12 \text { months. }\end{array}$ & $\begin{array}{c}\frac{\text { Implantation }}{\text { Bilateral }} \\
\frac{\text { Stimulation }}{\text { Monopolar }} \\
\frac{\text { Frequency }}{130 \mathrm{~Hz}} \\
\frac{\text { Amplitude }}{3-4.5 \mathrm{~V}} \\
\frac{\text { Pulse Width }}{60 \mu \mathrm{s}}\end{array}$ & $\begin{array}{c}\text { HRSD-17 } \\
\text { Object alternation } \\
\text { Test } \\
\text { Iowa gambling } \\
\text { task } \\
\text { Visual delayed } \\
\text { recall memory } \\
\text { Verbal delayed } \\
\text { memory } \\
\text { Verbal list learning } \\
\text { Stroop color-word }\end{array}$ & $\begin{array}{l}6 \text { months: four responded at the } \\
\text { end of DBS ** } \\
\text { General Neuropsychological } \\
\text { Performance: } \\
\text { Manual Motor Skills: Improved for } \\
\text { dominant and non-dominant hand } \\
\text { by } 12 \text { months. } \\
\text { Verbal learning: Restored } \\
\text { impairments in two patients at the } \\
\text { end of } 12 \text { months. } \\
\text { No significant correlations between } \\
\text { change in mood and } \\
\text { neuropsychological function at } 6 \\
\text { and } 12 \text { months. }\end{array}$ & N.A. \\
\hline Neimat et al. (2008) [74] & $\begin{array}{l}\text { Family history of severe } \\
\text { MDD. } \\
\text { Failed to respond to } \\
\text { antidepressants, adjuncts, } \\
\text { and ECT. } \\
\text { Relapsed after } \\
\text { ablative cingulotomy }\end{array}$ & 1 & 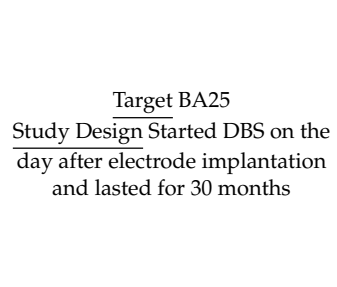 & $\begin{array}{l}\frac{\text { Implantation }}{\text { Bilateral }} \\
\frac{\text { Stimulation }}{\text { Monopolar }} \\
\frac{\text { Frequency }}{130 \mathrm{~Hz}} \\
\frac{\text { Amplitude }}{4.5 \mathrm{~V}} \\
\frac{\text { Pulse Width }}{60 \mu \mathrm{s}}\end{array}$ & HAMD-17 & $\begin{array}{l}\text { HAMD-17 score decreased from } 19 \\
\text { before surgery to } 8 \text { at } 6 \text { months } \\
\text { after DBS. } \\
\text { Sustained remission until the end } \\
\text { of DBS study (scored 7) }\end{array}$ & N.A. \\
\hline
\end{tabular}


Table 1. Cont.

\begin{tabular}{|c|c|c|c|c|c|c|c|}
\hline Authors & Main Inclusion Criteria & No. of Patients & $\begin{array}{c}\text { Stimulation Target \& DBS } \\
\text { Design }\end{array}$ & Stimulation Parameters & $\begin{array}{c}\text { Clinical } \\
\text { Evaluation }\end{array}$ & Major Outcomes & Adverse Effects \\
\hline Mayberg et al. (2005) [19] & $\begin{array}{l}\text { Current MDE } \geq 12 \text { months, } \\
\text { HRSD-17 score } \geq 20, \\
\text { Non-responsive (NR) } \geq 4 \\
\text { antidepressant therapies. }\end{array}$ & 6 & $\begin{array}{c}\text { Target BA25 } \\
\text { Study Design } \\
\text { 1-5 min-off stimulation in } \\
\text { acute DBS for } \\
5 \text { days postoperative. } \\
\text { Chronic DBS for } 6 \text { months after } \\
\text { pulse generator was implanted } \\
\text { and optimized for } 4 \text { wks }\end{array}$ & $\begin{array}{l}\text { Implantation } \\
\text { Bilateral Stimulation } \\
\text { Monopolar } \\
\text { Acute: } \\
\text { Frequency } \\
\frac{10-130 \mathrm{~Hz}}{\text { Amplitude }} \\
\frac{\text { A.0-9.0V }}{0.0-9} \\
\frac{\text { Pulse Width }}{30-250 \mu \mathrm{\mu s}} \\
\text { Chronic: } \\
\frac{\text { Frequency }}{130 \mathrm{~Hz}} \\
\frac{\text { Amplitude }}{4 \mathrm{~V}} \\
\frac{\text { Pulse Width }}{60 \mu \mathrm{s}}\end{array}$ & $\begin{array}{c}\text { HDRS-17, } \\
\text { MADRS, } \\
\text { CGI, } \\
\text { Positive and } \\
\text { Negative Affective } \\
\text { Scale. }\end{array}$ & $\begin{array}{l}\text { Acute effects: Sudden feeling } \\
\text { oc calmnness } \\
\text { Chronic effects: five patients } \\
\text { responded }{ }^{* *} \text { after } 2 \text { month DBS. } \\
\text { Response maintained in four } \\
\text { patients at the end of } 6 \text { month DBS. } \\
\text { Three patients achieved remission } \\
\text { व or near remission at the end of } 6 \\
\text { month DBS. }\end{array}$ & $\begin{array}{l}\text { Mild } \\
\text { Lightheadedness; } \\
\text { Psychomotor slowing } \\
\text { Skin infection; } \\
\text { Skin erosion. }\end{array}$ \\
\hline
\end{tabular}

* $\geq 40 \%$ reduction in MADRS and average GAF in months $4-6$ not worse than baseline; ${ }^{* *} \geq 50 \%$ reduction in HRSD-17 (HAMD-17) score from baseline; *** $\geq 40 \%$ reduction in HRSD-17

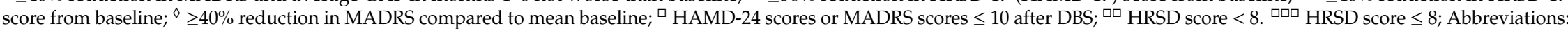
ATHF = Anti-depressant Treatment History Form, BA25 = Brodmann Area 25, BDI/-II = Beck Depression Inventory/-II, CGI, PGI, CANTAB = Clinician and Patient Global Impression of Severity and Improvement (CGI; PGI) and cognitive function (CANTAB); CVLT = California verbal learning test, DBS = deep brain stimulation, DWI = Diffusion-weighted imaging, ECG $=$ electrocardiogram, ECT = electroconvulsive therapy, EDA = electrodermal activity, EEG = electroencephalography, GAF = Global assessment function, HAM-A = Hamilton Anxiety Rating Scale, HRSD-17/HDRS-17 = Hamilton Rating Scale for Depression/ Hamilton Depression Rating Scale, (f)MRI = (functional) magnetic resonance imaging; MADRS/MARDS = Montgomery-Asberg Depression Rating Scale, MAOI = monoamine oxidase inhibitors, MDD = major depressive disorder, MDE $=$ major depressive episodes, MMSE $=$ Mini-Mental State Examination, NSAID = non-steroidal anti-inflammatory drug, NR = non-responsive, PET = positron emission tomography, QIDS/-SR = Quick Inventory of Depressive Symptomatology/-self report, Q-LES-Q = Quality of Life and Satisfaction Questionnaire, $\mathrm{SCC}=$ subcallosal cingulate, $\mathrm{SCG}=$ subcallosal cingulate gyrus, $\mathrm{SCR}=$ skin conductance response, $\mathrm{QOL}=\mathrm{Quality}$ of Life Enjoyment and Satisfaction Questionnaire, TRD = treatment-resistant depression, WSAS = Work and Social Adjustment Scale. 
Table 2. Summary of response and remission rates from clinical studies.

\begin{tabular}{|c|c|c|c|c|c|c|c|c|}
\hline \multirow[b]{2}{*}{ Authors } & \multicolumn{2}{|c|}{$\leq 6$ months } & \multicolumn{2}{|c|}{ 6-12 months } & \multicolumn{2}{|c|}{ 12-24 months } & \multicolumn{2}{|c|}{$\geq 24$ months } \\
\hline & $\begin{array}{c}\text { Response } \\
(\%)\end{array}$ & $\begin{array}{l}\text { Remission } \\
(\%)\end{array}$ & $\begin{array}{c}\text { Response } \\
(\%)\end{array}$ & $\begin{array}{c}\text { Remission } \\
(\%)\end{array}$ & $\begin{array}{c}\text { Response } \\
(\%)\end{array}$ & $\begin{array}{l}\text { Remission } \\
(\%)\end{array}$ & $\begin{array}{c}\text { Response } \\
(\%)\end{array}$ & $\begin{array}{c}\text { Remission } \\
(\%)\end{array}$ \\
\hline Sankar et al. 2020 [40] & NA & NA & NA & NA & NA & NA & NA & NA \\
\hline Riva Posse et al. (2019) [41] & NA & NA & NA & NA & NA & NA & NA & NA \\
\hline Eitan et al. (2018) [42] & NA & NA & NA & NA & 44.4 & NA & NA & NA \\
\hline Merkl et al. (2018) [43] & 37.5 & 12.5 & 43 & 14.2 & 33 & 33 & 33 & NA \\
\hline Howell et al. (2018) [44] & - & - & 33.3 & 66.7 & - & - & - & - \\
\hline Waters et al. (2018) [45] & NA & NA & NA & NA & NA & NA & NA & NA \\
\hline Smart et al. (2018) [46] & - & - & 78.5 & - & - & - & - & - \\
\hline Choi et al. (2018) [47] & NA & NA & NA & NA & NA & NA & NA & NA \\
\hline Conen et al. (2018) [48] & - & - & 28.6 & 42.9 & - & - & - & - \\
\hline Holtzheimer et al. (2017) [15] & $\begin{array}{c}22 \\
20 \text { (sham) }\end{array}$ & $\begin{array}{c}10 \\
7 \text { (sham) } \\
\end{array}$ & $\begin{array}{c}28 \\
30 \text { (sham) }\end{array}$ & $\begin{array}{c}12 \\
7 \text { (sham) }\end{array}$ & $\begin{array}{c}54 \\
52 \text { (sham) } \\
\end{array}$ & $\begin{array}{c}17 \\
20 \text { (sham) }\end{array}$ & $\begin{array}{c}48 \\
44 \text { (sham) }\end{array}$ & $\begin{array}{c}25 \\
12 \text { (sham) }\end{array}$ \\
\hline McInerney et al. (2017) [14] & - & - & 55 & - & - & - & - & - \\
\hline Riva-Posse et al. (2018) [49] & 72.7 & 54.5 & 81.8 & 54.5 & - & - & - & - \\
\hline Tsolaki et al. 2017 [50] & 50 & - & - & - & - & - & - & - \\
\hline Accolla et al. (2016) [51] & - & - & - & - & 79 & 20 & - & - \\
\hline Richieri et al. (2016) [52] & $\begin{array}{l}100 \\
\text { (Case } \\
\text { Study) }\end{array}$ & - & - & - & - & - & - & - \\
\hline Hilimire et al. (2015) [53] & NA & NA & NA & NA & NA & NA & NA & NA \\
\hline Martin-Blanco et al. (2015) [54] & NA & NA & NA & NA & NA & NA & NA & NA \\
\hline Puigdemont et al. (2015) [55] & - & 80 & - & - & - & - & - & - \\
\hline Serra-Blasco et al. (2015) [56] & - & - & - & - & $\begin{array}{l}75 \text { (F.E.) } \\
87 \text { (TRD) }\end{array}$ & - & - & - \\
\hline Choi et al. 2015 [57] & NA & NA & NA & NA & NA & NA & NA & NA \\
\hline Sun et al. 2015 [58] & NA & NA & NA & NA & NA & NA & NA & NA \\
\hline $\begin{array}{l}\text { Perez-Caballero et al. } \\
\text { (2014) [59] }\end{array}$ & 50 & - & - & - & - & - & - & - \\
\hline Merkl et al. (2013) [60] & - & - & - & 30 & - & - & - & - \\
\hline Ramasubbu et al. (2013) [61] & 50 & - & - & - & - & - & - & - \\
\hline Torres et al. (2013) [62] & $\begin{array}{c}100 \\
\text { (2 Case } \\
\text { Studies) }\end{array}$ & - & $\begin{array}{c}100 \\
\text { (2 Case } \\
\text { Studies) }\end{array}$ & - & $\begin{array}{c}100 \\
\text { (2 Case } \\
\text { Studies) }\end{array}$ & - & $\begin{array}{c}100 \\
\text { (2 Case } \\
\text { Studies) }\end{array}$ & - \\
\hline Broadway et al. (2012) [63] & 50 & - & - & - & - & - & - & - \\
\hline Hamani et al. (2012) [64] & - & $\begin{array}{c}100 \\
\text { (Case } \\
\text { Study) }\end{array}$ & $\begin{array}{c}100 \\
\text { (Case } \\
\text { Study) }\end{array}$ & - & - & - & - & - \\
\hline Holtzheimer et al. (2012) [65] & 18 & 41 & 36 & 36 & 58 & 92 & - & - \\
\hline Lozano et al. (2012) [66] & 57 & - & 48 & - & 62 & - & - & - \\
\hline Puigdemont et al. (2012) [67] & 37.5 & 37.5 & 87.5 & 37.5 & 62.5 & 50 & - & - \\
\hline Kennedy et al. (2011) [18] & - & - & 62.5 & - & 46.2 & - & 75 & - \\
\hline Guinjoan et al. (2010) [68] & $\begin{array}{c}100 \\
\text { (Case } \\
\text { Study) }\end{array}$ & - & $\begin{array}{l}100 \\
\text { (Case } \\
\text { Study) }\end{array}$ & - & $\begin{array}{l}100 \\
\text { (Case } \\
\text { Study) }\end{array}$ & - & - & $\begin{array}{c}100 \\
\text { (Case } \\
\text { Study) }\end{array}$ \\
\hline $\begin{array}{l}\text { Holtzheimer and Mayberg } \\
\text { (2010) [69] }\end{array}$ & $\begin{array}{c}100 \\
\text { (Case } \\
\text { Study) }\end{array}$ & - & $\begin{array}{c}100 \\
\text { (Case } \\
\text { Study) }\end{array}$ & - & $\begin{array}{c}100 \\
\text { (Case } \\
\text { Study) }\end{array}$ & - & $\begin{array}{l}100 \\
\text { (Case } \\
\text { Study) }\end{array}$ & - \\
\hline Hamani et al. (2009) [70] & - & - & 55 & - & - & - & - & - \\
\hline Puigdemont et al. (2009) [71] & $\begin{array}{c}100 \\
\text { (Case } \\
\text { Study) }\end{array}$ & - & $\begin{array}{c}100 \\
\text { (Case } \\
\text { Study) }\end{array}$ & - & - & - & - & - \\
\hline Lozano et al. (2008) [72] & 35 & 10 & 60 & 35 & - & - & - & - \\
\hline McNeely et al. (2008) [73] & 66 & NA & - & - & - & - & - & - \\
\hline Neimat et al. (2008) [74] & $\begin{array}{c}100 \\
\text { (Case } \\
\text { Study) }\end{array}$ & NA & - & - & - & - & $\begin{array}{c}100 \\
\text { (Case } \\
\text { Study) }\end{array}$ & - \\
\hline Mayberg et al. (2005) [19] & 66 & 50 & - & - & - & - & - & - \\
\hline Average & $63.8 \%$ & $43.9 \%$ & $66.5 \%$ & $36.5 \%$ & $69.3 \%$ & $42.4 \%$ & $76 \%$ & $62.5 \%$ \\
\hline Range & $18-100$ & $10-100$ & $28.6-100$ & $12-66.7$ & $33-100$ & $17-92$ & 33-100 & $12-100$ \\
\hline
\end{tabular}

The first evidence-based clinical study on SCC-DBS was published by Mayberg et al. in 2005 [19]. Among six patients with an average of 5.6 years of major depressive episode (MDE), four responded to 
the treatment, but three remitted or nearly remitted during the stimulation, even without changing medications. The authors found that the metabolic activity in the SCC normalized from a hyperactive state and was accompanied by reduced local blood flow as detected by Positron Emission Tomography (PET) [19]. In a study from 2003 to 2006 by Lozano et al. on chronic DBS in 20 patients with an average of 6.9 years of current MDE, 11 patients responded, but seven remitted [72], which was similar to the response and remission rates of Mayberg et al. In a 3.5-year follow-up study, the response rate was consistent across time points, but the remission rate increased from $18.8 \%$ to $42.9 \%$ at the last visit [18]. Both studies reported changes in structures distal to SCC after DBS, which explains the persistent response throughout the DBS treatments [18,72].

In a case report by Neimat et al., a 55-year-old female TRD patient who relapsed after a subgenual cingulotomy, achieved sustained remission for up to 30 months with SCC-DBS treatment [74]. In a case reported by Guinjoan et al. in 2010, a 60-year-old male TRD patient responded to unilateral SCC-DBS in the right hemisphere, but unilateral stimulation in the left hemisphere worsened his mood. This is in line with the asymmetrical response to antidepressants in the SCC region. However, the authors noted a further study was needed with more patients to validate the effects of unilateral stimulation on mood enhancement [68].

Similarly, in a preliminary study in 2012 by Puigdemont et al. on eight patients with an average of 6.3 years of current MDE, they found that five patients responded at the end of the 12-month DBS, but three out of four final remitters remitted after 3 months of DBS [67]. Their cognitive functions were not exacerbated and their memory functions were actually improved in cognitive assessments in 2015 [56]. Concurrently, a clinical study conducted in three different medical centers also reported similar efficacies of SCC-DBS, suggesting that DBS has reliable stimulation effects. Among 21 patients with an average of 5 years of current MDE, 13 responded to the treatment and the rest performed better than at baseline by the end of the study, although one patient committing suicide by medication overdose [66].

\subsection{Remission Rates}

Some previous studies reported higher initial response and/or remission rates compared to more recent studies $[19,67,72]$. In the study by Perez-Caballero et al., they suggested that electrode insertion-induced inflammation could affect response and remission rates. Four of the eight recruited patients took non-steroidal anti-inflammatory drugs (NSAIDs), which resulted in a diminished antidepressant response toward DBS, whereas the other four not taking NSAIDs gradually responded and remitted. The authors also analyzed the role of inflammation in the early DBS response in rats [59], which is discussed in a later section of this review. A later study in 2015 by Puigdemont et al. reported that remission was maintained in four out of five remitted patients in the 3-month active stimulation group, whereas only two patients remitted in the sham stimulation group. They concluded that continuous active stimulation was important in maintaining the therapeutic effect [55]. This was supported by an earlier case of a 27-year-old patient on DBS for 2 years whose symptoms worsened due to battery depletion, but improved again upon battery replacement [69].

Table 2 reflects the different response and remission rates, at 6-month intervals, across the duration of the studies in Table 1. This reporting allows for a cursory longitudinal tracking in understanding how response and remission may change with time. Among the reviewed studies on DBS, the response rate ranged from $18 \%$ to $87.5 \%$ and remission rate ranged from $10 \%$ to $92 \%$ (excluding all case studies) across the different time points (see Table 2), which were comparable to earlier clinical studies $[19,72]$. However, large-scale controlled trials are needed to further validate the efficacy of DBS in patients with TRD. Some predictive markers discovered in these studies could facilitate the selection of more responsive patients and increase the safety of DBS. A noteworthy study by Holtzheimer and Mayberg demonstrated some changes in the response and remission rates with DBS [69]. The authors noted that several months after a response and/or remission in their depressive symptoms, worsening of symptoms was temporarily observed at 16 weeks. They attributed the temporary worsening of symptoms to 
the difficulty of some patients reintegrating into society. In an earlier study by Lozano et al. in 2008, they also observed a similar occurrence at 4 months. These findings highlight the complexity of treating neuropsychiatric diseases, as the recovery periods are not always consistent and can be affected by different factors.

\subsection{Significant Challenges in the Development of SCC-DBS}

A larger study that aimed to recruit 201 patients was conducted by Holtzheimer et al. in 2017 to further validate the therapeutic effects of DBS [15]. A futility analysis conducted after 90 patients had been recruited showed no significant differences between the DBS and sham groups, leading to the early termination of the study. During a 6-month double-blind trial, no significant differences were found in the response of the DBS group compared to the sham group. However, among 77 patients that received subsequent open-label DBS for up to 2 years, 38 responded and 20 remitted. Holtzheimer et al. offered several explanations for the observed result. First, the patients selected for the study had an average current episode duration of around 12 years, whereas most studies recruited patients with an average current depressive episode of about 3-5 years. Holtzheimer also posited the possibility of suboptimal contact during the first 12 months, further affecting the results. This landmark paper was initially thought to be the death knell for DBS as a treatment for TRD. However, a summit of key academics within the field determined that DBS protocols required further modification and patient recruitment needed refining to better assess the therapeutic effects of DBS for TRD [75]. Considering that multiple other studies showed the efficacy and effectiveness of DBS for TRD, the conference considered several possibilities for the discrepancies in the findings, some conclusions were that DBS was initiated too early before optimal targeting was secured, a lack of specificity and standardization in the improvement of symptoms, high placebo effects typically seen in the treatment of psychiatric disorders, and study design. The heterogeneity of the symptoms of the disease was also emphasized, which suggested that different circuitry might be involved in different individuals. The key conclusions from the summit included that patient selection should be better and more refined, study designs should be either fast to fail or fast to succeed, registries should be established for better subject tracking, and longitudinal data should be collected. The paper stressed that the complexities of the disease were real and better experimental designs were needed to truly reflect the effects of DBS as a treatment for TRD for a better response and remission rate and to allow the elucidation of the mechanistic role of DBS.

\subsection{Adverse Effects}

The safety of SCC-DBS was subsequently assessed following the initial results of the efficacy of DBS in treating TRD. In 2008, McNeely et al. conducted a trial on six patients with an average of 5.6 years of current MDE. They found that mood was significantly improved during the 1-year DBS treatment without serious cognitive deterioration [73]. Moreines et al. found that DBS treatment in both unipolar and bipolar TRD patients with at least 2 years of current MDE improved executive functions and stabilized their memory [76]. Similarly, SCC-DBS for 6 months followed by depression treatment in patients with MDD, who had increased negative emotional processing and/or reduced positive emotions, resulted in reduced negative emotional bias [53]. Martín-Blanco et al. reported that a 52-year-old female had a seizure after 5 weeks of DBS. As severe MDD may predispose patients to seizures, the authors recommended that patients should be evaluated for seizures before administering DBS and parameters might need to be adjusted to within safe ranges [52]. In a study in 2017 by McInerney et al. on 20 unipolar TRD patients with an average of 6.9 years of MDE, they reported that 11 patients responded at the end of the 12-month DBS without further deterioration of cognitive functions. They also found a correlation between verbal fluency and mood improvement, which could be predictive of the DBS response [14]. The side effects reported in this review range from mild symptoms, such as headaches, dizziness and gastrointestinal irritation [43,66], to more severe effects including suicidal ideation and device malfunction $[15,67]$. This reporting should not discourage the development of therapies. Indeed, many treatments including serotonin-selective reuptake inhibitors 
have severe side effects, including increased fractures and suicidal ideations $[77,78]$. In the study of therapies, it is important to report these side effects and to note that these therapies are administered by a professional, whose role is to detect and modulate the therapies accordingly.

\subsection{Stimulation Parameters}

Several studies have attempted to optimize the parameters of DBS for treating mood disorders. As previously mentioned, Eitan et al. reported that high-frequency stimulation (HFS) was more effective at lowering MADRS scores compared with low frequency stimulation [42]. Indeed, the most commonly used stimulation frequency was in the high frequency range of $130-135 \mathrm{~Hz}$, although some studies have tested frequencies between 5 and $185 \mathrm{~Hz}[19,61]$ (see Table 1). The pulse width used in DBS also varied greatly across studies. In a study by Ramasubbu et al., they found that a long pulse width of 180-270 $\mu$ s was effective [61]. However, this study also reported that DBS with a long pulse width caused patients to experience stimulation-induced insomnia, anxiety, confusion, and drowsiness. Previous studies by Lozano et al. and Holtzheimer et al. demonstrated that shorter pulse widths of 30-60 $\mu$ s led to clinical improvements in depression symptoms without these side effects $[66,72]$. Indeed, Ramasubbu et al. suggested that longer pulse widths with lower amplitudes and shorter pulse widths with higher amplitudes could produce comparable therapeutic benefits. The amplitude of the stimulating current used in DBS to elicit a therapeutic response also tended to vary across studies. The amplitude is the first parameter to be adjusted when patients do not respond to the treatment. Among 38 clinical studies, the overall current range was $2-8 \mathrm{~mA}$ and voltage range was $2.5-10.5 \mathrm{~V}$. The variability in the amplitude underscores the personalized nature of DBS, which requires specific adjustments to achieve individual therapeutic effects.

\subsection{Electrode Implantation}

Several clinical DBS studies have also tried to improve the accuracy of electrode implantation in order to precisely target regions of interest. The pioneering work by Mayberg et al. used PET scans of pathological glucose metabolism to guide the electrode implantation. Riva-Posse et al. used individualized tractography maps based on a group connectome blueprint of past responders to DBS to identify optimal target regions for electrode implantation [49]. Riva-Posse et al. used a four-bundle white matter blueprint, which resulted in good clinical outcomes in eight out of 11 patients, which suggests that the use of this method could improve the precision of implantation. Similarly, Tsolaki et al. investigated the use of FMRIB Software Library (FSL) probabilistic tractography in SCC-DBS [50]. Several studies have used other methods to try to specify the optimal stimulation points. Choi et al. investigated the best contact positions that elicited the best response during intraoperative testing [57]. They used diffusion-directed magnetic resonance imaging and patient-specific tractography maps to guide the implantation. They also used fiber tract probabilistic tractography to determine the putative fiber tract activation in patients, which was used to guide the electrode implantation for the best response, rather than the salient response. Contacts in the left hemisphere were found to produce the best consistent intraoperative response to DBS in seven out of nine patients at 6 months. Smart et al. validated this result in their study using local field potentials following unilateral HFS-DBS [46]. They found that left-sided stimulation evoked broadband effects, compared with right sided stimulation, which evoked only beta and gamma bands. Additionally, a decrease in theta bands was consistently accompanied by behavioral improvements. They concluded that the precision of electrodes in the left-hemisphere was more important and instructive than in the right hemisphere. In contrast, Guinjoan et al. and Howell et al. found that right hemisphere targets were critical for behavioral improvements [44,68]. Guinjoan et al. showed that right unilateral DBS could reverse and remit a patient's worsening mood induced by left unilateral DBS. Howell et al. showed that right cingulate bundle activation beyond a threshold could protract the recovery. Further research is needed to elucidate the differences in these studies. 


\subsection{Other Responses to DBS}

With regard to other potential responses to DBS, recent studies have attempted to characterize non-behavioral evoked responses. Conen et al. identified higher rCBF in patients at baseline and during DBS therapy compared to non-remitters and non-responders [48]. Riva-Posse et al. observed autonomic changes in responders undergoing DBS [41]. In a study by Smart et al., assessing the efficacy of DBS, they found consistent changes in left theta local field potentials, which could provide another consistent parameter to monitor. Based on this finding, they proceeded to adjust the contacts for one non-responder, who was able to achieve a response by the end of the study. In a study by Sankar et al. on responders and non-responders who had previously undergone SCG-DBS, they found that both groups had significant volume differences in the left and average SCG; in the right and average amygdala; and in the left, right, and average thalamus (Sankar et al. 2020). Additionally, non-responders had significantly greater grey matter volume compared to responders and a greater grey to white matter ratio. This important information provides yet more criteria for assessing if a patient might respond to DBS. Expanding the breadth of data obtained during clinical trials has the potential to advise clinicians on the efficacy of DBS, and to help predict non-responders and adjust the stimulation parameters. This will improve patient welfare and allows for a more accurate examination of the mechanisms of DBS in improving depressive-like symptoms.

Furthermore, it would be prudent to use preclinical results to advise clinical cases. In a previous preclinical study, Hamani et al. reported that DBS supplemented with tranylcypromine increased the antidepressant-like response in animals by $20 \%-30 \%$ compared to either treatment alone. They later reported on a patient who relapsed after 4 years of remission following DBS treatment [64]. Based on their previous work, they administered tranylcypromine before the DBS treatment, which allowed the patient to enter remission again.

\section{Preclinical Studies of Electrical Stimulation in the Medial Prefrontal Cortex in Rodents}

Following the success of a number of preliminary clinical studies, several preclinical studies were conducted to investigate the antidepressant-like effects of DBS [79]. The MPFC in rats is widely regarded to be homologous to the SCC in humans. The mPFC together with the amygdala, hippocampus, and hypothalamus controls the stress response, autonomic functions, and cognition in rats [80-83]. Using PET imaging, glucose metabolism was observed to normalize in the mPFC from a hyperactive state following DBS, which was similarly observed in the SCC after $1 \mathrm{~h}$ of DBS [84]. However, the homology between subdivisions of MPFC and SCC is still under debate. The vmPFC can be further subdivided into the infralimbic (IL) and prelimbic (PrL) regions. Although there are overlaps, the IL and PrL innervate different regions to different extents, including the lateral hypothalamus, dorsal raphe nucleus, and amygdala, among efferent regions [85-87]. The PrL has been shown to innervate to important limbic regions associated with SCC projections [87]. Meanwhile, the infralimbic cortex (IL) is believed to be structurally homologous based on comparisons involving tractography analysis [88-90]. Some assert that the whole ventromedial prefrontal cortex (vmPFC) is homologous to the SCC [91,92]. Others assert that the vmPFC is functionally distinct from the dorsal medial prefrontal cortex [70,91]. Nevertheless, the vmPFC is generally regarded as homologous to BA25, although a thorough understanding of specific correlations remains to be seen. Table 3 lists 29 preclinical studies on the effects of vmPFC-DBS on animal behaviors.

\section{1. vmPFC Stimulation}

Hamani et al. published the first preclinical study of vmPFC-DBS in rats in 2010. They used the forced swim test (FST), which models "helplessness" in animals including anxiolytic-like and anti-anhedonic-like behavior. They found DBS reduced the immobility score in FST, indicating antidepressant-like effects. The authors attributed the behavioral changes to serotonergic function in the dorsal raphe nucleus (DRN) as lesions in this structure abolished the behavioral effects 
in FST [91]. Another animal study in 2012 found the optimal stimulation frequency and amplitude of vmPFC-DBS was $130 \mathrm{~Hz}$ and $200 \mu \mathrm{A}$ that produced anti-anhedonic-like effects and produced a charge density similar to DBS in humans [93]. They found a lesion in the DRN abolished the higher sucrose consumption due to DBS, even with a normal hippocampal brain-derived neurotrophic factor (BDNF) profile. They postulated that an interaction between BDNF and neurochemical substances potentiated the antidepressant-like response [92]. The anti-anhedonic-like effects of DBS were also supported in studies by Rea et al. and Edemann-Callesen et al. They conducted an intracranial, self-stimulation paradigm in Flinders sensitive line and Flinders resistant line rats to assess reward-seeking behaviors, which demonstrated that the anti-anhedonic-like effect of vmPFC-DBS was independent of the dopaminergic reward system [94,95]. Bruchim-Samuel et al. reported that modulation of the ventral tegmental area could prolong the behavioral changes. They found that intermittent acute patterned stimulation administered to the ventral tegmental area of Flinders sensitive line rats resulted in antidepressant-like and anti-anhedonic-like behaviors [96]. Strikingly, a study by Bregman et al. in 2018 found that the antidepressant-like effect of DBS was serotonin transporter-independent. This could be of benefit to some patients with a mutated serotonin transporter-promoter gene (5-HTTLPR), which underlies the poor response to conventional selective serotonin re-uptake inhibitors that target serotonin transporters [97].

Beside changes in neurochemical and neurotrophin profiles, neuroplasticity changes induced by DBS have also been investigated. For instance, Bambico et al. reported increased hippocampal neurogenesis and BDNF levels after vmPFC-DBS, which led to anti-anhedonic-like behaviors, but was not sufficient for an overall antidepressant-like effect [98]. Correspondingly, Liu et al. found a correlation between vmPFC-HFS and hippocampal neurogenesis and improvements in short- and long-term memory in middle-aged rats. This suggests that DBS has therapeutic potential in age-dependent memory deficits [99].

\subsection{Other Brain Targets}

As preclinical studies have progressed, several brain targets of DBS have been established. Hamani et al. in 2014 demonstrated that DBS in the nucleus accumbens induced a similar antidepressant-like effect to DBS in the vmPFC, even though the stimulations modulated different circuits. This may contribute to more customized stimulation targeting based on the patient's symptoms [100]. Bregman et al. reported that the HFS of the medial forebrain bundle induced antidepressant-like behaviors in the FST [101]. Interestingly, this antidepressant effect was not mediated by increases in either serotonin or dopamine release in the nucleus accumbens. Lim et al. in 2015 emphasized that only HFS of the vmPFC led to anti-anhedonic-like effects and these pronounced antidepressant-like effects were induced by modulating the activity of serotonergic neurons in the DRN [102]. However, the authors did not investigate the effects of different stimulation parameters on depressive-like behaviors in various DBS targets. The study by Etiévant et al. supported the modulation of DRN by DBS and added that glial integrity was a prerequisite to the antidepressant-like outcome [103]. In another study, mice subjected to chronic social defeat stress were administered 7 days of 5-h vmPFC-DBS, which resulted in increased social interactive behavior accompanied by DRN modulation [104]. Interestingly, a recent study demonstrated that the potentiation of the anxiolytic response to vmPFC stimulation was associated with exposure to an enriched environment. This indicates that an enriched living environment can facilitate the beneficial effects of DBS intervention [105]. Creed et al. conducted DBS on the entopeduncular and the subthalamic nuclei to compare antidepressant-like effects [106]. Chronic Subthalamic nucleus DBS was reported to impair performance in the learned helplessness task, with no significant effects in anxiety tests. These results were associated with decreased hippocampal BDNF and TrkB mRNA. Interestingly, entopeduncular nucleus DBS did not increase depressive-like behavior in the learned helplessness task, indicating a superior target over the subthalamic nucleus for the treatment of depressive-like behaviors. Meng et al. reported reductions in depressive-like behaviors in animals stimulated in 
the lateral habenula; this observation was associated with elevations in dopamine, norepinephrine, and serotonin in both blood serum and in the hippocampus [107].

\section{3. vmPFC-Linked Modulation of Other Structures}

Other structures have been found to be modulated by vmPFC-DBS. For example, Lim et al. reported that activation of the medial subthalamic nucleus contributed to antidepressant-like behavior [108]. In a rat model of post-traumatic stress disorder, IL-DBS reduced firing in the basolateral amygdala, which attenuated fear and produced a slight anxiolytic-like effect [109]. A recent study showed that DBS resulted in elevated spontaneous firing of noradrenergic locus coeruleus neurons and strengthened the coherence between the prefrontal cortex and locus coeruleus. The latter was protective against stress and was responsible for the antidepressant-like effect seen in FST [110]. On the other hand, Insel et al. reported that there was reduced communication between IL and ventral hippocampus in rats after 10 days of 8-h IL-DBS and such coherence was higher in depressed subjects [111]. Jiménez-Sánchez et al. in 2016 reported two studies on acute IL-DBS in naive and olfactory bulbectomized rat models. In naive animals, IL-DBS induced antidepressant-like behaviors and increased prefrontal glutamate efflux, which activated the $\alpha$-amino-3-hydroxy-5-methyl-4-isoxazolepropionic acid receptor (AMPAR) to modulate DRN output [81]. In olfactory bulbectomized rats, similar changes were noted in the prefrontal serotonergic and glutamatergic output with the activation of AMPAR and antidepressant-like behaviors [33].

\subsection{Synergism with Other Treatments}

Antidepressant-like effects in different DBS paradigms are leading to some advancements in the field. One such investigation by Laver et al. in 2014 examined the use of augmentation agents such as buspirone, risperidone, and pindolol to enhance DBS efficacy. However, these agents did not increase the antidepressant response of the rats receiving DBS treatment, when compared to those co-administered monoamine oxidase inhibitors in previous studies [64,112]. It is possible that a response may become evident in clinical trials. Perez-Caballero et al. in 2014 reported an interesting early response to stimulation, in which sham-treated rats had reduced immobility and increased swimming in FST at weeks 1 and 2, but not at week 6 post treatment. They reasoned that this was caused by insertion-induced inflammation as pretreatment by indomethacin reduced the expression of pro-inflammatory mediators (TNF- $\alpha, \mathrm{COX} 1, \mathrm{COX} 2$ ) and reversed the antidepressant-like behaviors in sham-treated animals [59]. Rummel et al. in 2016 reported that chronic continuous HFS did not have more benefits than chronic intermittent stimulation in treatment-resistant rats with congenitally learned helplessness [113].

\subsection{Other Biological Parameters Modulated}

Similar to the research direction of clinical studies, preclinical studies have also attempted to characterize other biological parameters of DBS, including more precise electrode implantation. Lehto et al. characterized real-time fMRI responses in the brain following DBS, and found strong connectivity between the vmPFC and amygdala, which validated vmPFC as a target region [114]. Perez-Cabalerro et al. used PET scans to study the immediate effects of electrode implantation. They found that metabolism was decreased locally (vmPFC), but was increased in ventral regions, including dorsal and ventral hippocampus, piriform and insular cortex, nucleus accumbens, ventral tegmental area, ventral pallidum, hypothalamus, and the preoptic area [115]. This was in agreement with other studies on the effect of DBS on depressive-like behavior, but it is noteworthy to see these effects simply via electrode insertion. 
Table 3. A list of preclinical studies on deep brain stimulation of the medial prefrontal cortex in rodents.

\begin{tabular}{|c|c|c|c|c|c|c|}
\hline Authors & Target & Animal & Animal Models \& DBS Design & Stimulation Parameters & Behavioral Tests & Outcomes \\
\hline \multirow{3}{*}{ Jia et al. (2019) [116] } & \multirow{3}{*}{ vmPFC } & \multirow{3}{*}{$\begin{array}{l}\text { Sprague-Dawley rats, } \\
\text { male. }\end{array}$} & \multirow{3}{*}{$\begin{array}{l}\text { CUS animal model. } \\
\text { Open field test and forced swim test } \\
\text { before DBS. }\end{array}$} & \multirow{3}{*}{$\begin{array}{c}\text { Unipolar } \\
\text { High Frequency } \\
\text { Frequency: } 130 \mathrm{~Hz} \\
\text { Amplitude: } 100 \mu \mathrm{A} \\
\text { Pulse Width: } 90 \mu \mathrm{s} \\
\text { Low Frequency } \\
\text { Frequency: } 20 \mathrm{~Hz} \\
\text { Amplitude: } 400 \mu \mathrm{A} \\
\text { Pulse Width: } 0.2 \mu \mathrm{s}\end{array}$} & Sucrose preference test & $\begin{array}{l}\text { CUS rats had a } \\
\text { lowered sucrose } \\
\text { preference compared } \\
\text { to control rats. }\end{array}$ \\
\hline & & & & & Open field test & $\begin{array}{l}\text { No significant } \\
\text { difference in } \\
\text { locomotion was } \\
\text { recorded between } \\
\text { CUS and } \\
\text { control groups. } \\
\end{array}$ \\
\hline & & & & & Forced swim test & $\begin{array}{c}\text { Both High- and } \\
\text { Low-Frequency } \\
\text { Stimulation reduced } \\
\text { immobility compared } \\
\text { to sham rats. }\end{array}$ \\
\hline \multirow[b]{2}{*}{ Papp et al. (2019) [117] } & \multirow[b]{2}{*}{ vmPFC } & \multirow[b]{2}{*}{$\begin{array}{l}\text { Wistar-Kyoto rats, male (DBS) } \\
\text { Wistar rats, male } \\
\text { [Venlafaxine(VFX)-treated] }\end{array}$} & \multirow[b]{2}{*}{$\begin{array}{l}\text { CMS animal model. } \\
\text { Two, 2-h DBS sessions were conducted, } \\
\text { one on the preceding evening and the } \\
\text { other on the following morning before } \\
\text { each sucrose intake test and the NORT } \\
\text { T1 session. }\end{array}$} & \multirow[b]{2}{*}{$\begin{array}{l}\text { Frequency: } 130 \mathrm{~Hz} \\
\text { Amplitude: } 250 \mu \mathrm{A} \\
\text { Pulse width: } 90 \mu \mathrm{s}\end{array}$} & Sucrose intake test & $\begin{array}{l}\text { During the first } 2 \mathrm{wks} \\
\text { of CMS, sucrose } \\
\text { intake decreased } \\
>50 \% \text { across groups. } \\
\text { VFX treatment } \\
\text { restored sucrose } \\
\text { intake levels. }\end{array}$ \\
\hline & & & & & $\begin{array}{l}\text { Novel object recognition } \\
\text { test }\end{array}$ & $\begin{array}{c}\text { Wistar Kyoto rats: } \\
\text { DBS rescued novel } \\
\text { object recognition tes } \\
\text { across all groups. } \\
\text { Wistar rats: VFX } \\
\text { rescued novel object } \\
\text { recognition test in } \\
\text { CMS animals } \\
\text { administered with D2 } \\
\text { antagonist, but not in } \\
\text { D2-administered } \\
\text { CMS animals. VFX } \\
\text { also did not rescue } \\
\text { groups administered } \\
\text { with D3 antagonist. }\end{array}$ \\
\hline Bhaskar et al. (2018) [105] & vmPFC & $\begin{array}{l}\text { Wistar rats, } \\
\text { male. }\end{array}$ & $\begin{array}{l}\text { Naïve animal model. } \\
\text { DBS for } 15 \text { min prior to and throughout } \\
\text { behavioral testing. }\end{array}$ & $\begin{array}{c}\text { Bipolar } \\
\text { Frequency: } 100 \mathrm{~Hz} \\
\text { Amplitude: } 200 \mu \mathrm{A} \\
\text { Pulse Width: } 100 \mu \mathrm{s}\end{array}$ & Home-cage emergence test & $\begin{array}{c}\text { Enriched } \\
\text { environment } \\
\text { potentiated the } \\
\text { efficacy of HFS on } \\
\text { reduced escape } \\
\text { latency time in the } \\
\text { Naiive animal model. }\end{array}$ \\
\hline
\end{tabular}


Table 3. Cont

\begin{tabular}{|c|c|c|c|c|c|c|}
\hline Authors & Target & Animal & Animal Models \& DBS Design & Stimulation Parameters & Behavioral Tests & Outcomes \\
\hline & & & & & Elevated plus maze & $\begin{array}{l}\text { HFS with an enriched } \\
\text { environment reduced } \\
\text { the anxiety index and } \\
\text { increased head dips. }\end{array}$ \\
\hline & & & & & $\begin{array}{c}\text { Novel object } \\
\text { recognition test }\end{array}$ & $\begin{array}{l}\text { No significant } \\
\text { difference. }\end{array}$ \\
\hline \multirow{2}{*}{ Bregman et al. (2018) [97] } & \multirow{2}{*}{ vmPFC } & \multirow{2}{*}{$\begin{array}{l}\text { SERT homozygous knockout } \\
\text { and wildtype mice, male. }\end{array}$} & \multirow{2}{*}{$\begin{array}{l}\text { Serotonin transporter (SERT) knockout } \\
\text { model. } \\
\text { DBS for } 4 \mathrm{~h} \text { before forced swim test and } \\
\text { open field test. }\end{array}$} & \multirow{2}{*}{$\begin{array}{l}\text { Bilateral Monopolar } \\
\text { Frequency: } 130 \mathrm{~Hz} \\
\text { Amplitude: } 100 \mu \mathrm{A} \\
\text { Pulse Width: } 90 \mu \mathrm{s}\end{array}$} & Forced swim test & $\begin{array}{c}\text { Both wild-type and } \\
\text { knockout-DBS mice } \\
\text { had reduced } \\
\text { immobility time } \\
\text { compared to sham. }\end{array}$ \\
\hline & & & & & Open field test & $\begin{array}{l}\text { Knockout-DBS mice } \\
\text { had lower locomotion } \\
\text { counts than sham and } \\
\text { wild-type mice. }\end{array}$ \\
\hline Lehto et al. (2018) [114] & $\mathrm{IL}$ & $\begin{array}{l}\text { Sprague-Dawley rats, } \\
\text { male. }\end{array}$ & $\begin{array}{l}\text { Naïve animal model. } \\
\text { All stimulation paradigms consisted of } \\
\text { three blocks of } 60 \mathrm{~s} \text { of rest and } 18 \mathrm{~s} \text { of } \\
\text { stimulation, ending with an additional } \\
\text { rest period, giving a total paradigm of } 4 \\
\text { min } 54 \mathrm{~s} .\end{array}$ & $\begin{array}{c}\text { Monopolar } \\
\text { Frequency: } 20 / 35 / 70 / 100 / 130 / 160 / 200 \mathrm{~Hz} \\
\text { tested in randomized order. } \\
\text { Amplitude: } 1.4-1.7 \mathrm{~mA} \text { distributed } \\
\text { equally among the three electrode } \\
\text { channels } \\
\text { Pulse Width: } 180-\mu \mathrm{s}\end{array}$ & N.A. & $\begin{array}{c}\text { fMRI conducted to } \\
\text { characterize changes } \\
\text { in the brain following } \\
\text { DBS. } \\
\text { IL-DBS at varying } \\
\text { stimulation } \\
\text { parameters } \\
\text { significantly triggered } \\
\text { the amygdala. } \\
\text { Orientation selective } \\
\text { stimulation was able } \\
\text { to recruit neuronal } \\
\text { pathways of distinct } \\
\text { orientations relative } \\
\text { to the position of the } \\
\text { electrode. }\end{array}$ \\
\hline \multirow[t]{2}{*}{ Papp et al. (2018) [118] } & \multirow[t]{2}{*}{ vmPFC } & \multirow[t]{2}{*}{$\begin{array}{c}\text { Wistar rats, } \\
\text { male. }\end{array}$} & \multirow{2}{*}{$\begin{array}{l}\text { CMS animal model. } \\
\text { Two, 2-h DBS sessions were conducted, } \\
\text { one on the previous evening and one the } \\
\text { next morning } 15 \text { min before each } \\
\text { behavioral test. }\end{array}$} & \multirow{2}{*}{$\begin{array}{c}\text { Bipolar } \\
\text { Frequency: } 130 \mathrm{~Hz}, \\
\text { Amplitude: } 250 \mu \mathrm{A}, \\
\text { Pulse Width: } 90 \mu \mathrm{s}\end{array}$} & Sucrose intake test & $\begin{array}{c}\text { DBS increased } \\
\text { sucrose intake across } \\
\text { all treatment groups, } \\
\text { except for } \\
\text { imipramine-treated } \\
\text { animals. } \\
\end{array}$ \\
\hline & & & & & Elevated plus maze & $\begin{array}{l}\text { DBS increased the } \\
\text { anxiolytic open arm } \\
\text { entries in all } \\
\text { treatment groups. }\end{array}$ \\
\hline
\end{tabular}


Table 3. Cont.

\begin{tabular}{|c|c|c|c|c|c|c|}
\hline Authors & Target & Animal & Animal Models \& DBS Design & Stimulation Parameters & Behavioral Tests & Outcomes \\
\hline & & & & & $\begin{array}{l}\text { Novel object recognition } \\
\text { test }\end{array}$ & $\begin{array}{l}\text { DBS rescued the } \\
\text { abolished novel object } \\
\text { recognition in CMS } \\
\text { sham-treated animals, } \\
\text { across all treatment } \\
\text { groups. }\end{array}$ \\
\hline \multirow[b]{3}{*}{$\begin{array}{l}\text { Perez-Caballero et al. } \\
\text { (2018) [115] }\end{array}$} & \multirow[b]{3}{*}{$\mathrm{IL}$} & \multirow[b]{3}{*}{$\begin{array}{l}\text { Wistar rats, } \\
\text { male. }\end{array}$} & \multirow[b]{3}{*}{$\begin{array}{l}\text { Six independent sets of animals using } \\
\text { naïve (unoperated controls) and DBS-off } \\
\text { animals. }\end{array}$} & \multirow[b]{3}{*}{ N.A. } & Paw-pressure test & $\begin{array}{l}\text { Ibuprofen, tramadol, } \\
\text { and morphine } \\
\text { significantly } \\
\text { increased the paw } \\
\text { withdrawal threshold } \\
\text { in naïve animals } \\
\text { relative to respective } \\
\text { vehicle alone, } \\
\text { demonstrating a clear } \\
\text { analgesic effect. }\end{array}$ \\
\hline & & & & & Open field test & $\begin{array}{c}\text { No analgesics altered } \\
\text { the motor activity of } \\
\text { rats. }\end{array}$ \\
\hline & & & & & Modified forced swim test & $\begin{array}{c}\text { Electrode } \\
\text { implantation induced } \\
\text { a significant reduction } \\
\text { in the immobility } \\
\text { scores of } \\
\text { vehicle-treated } \\
\text { animals. } \\
\text { Ibuprofen abolished } \\
\text { the } \\
\text { antidepressant-like } \\
\text { effect of electrode } \\
\text { implantation in the } \\
\text { modified forced swim } \\
\text { test, increasing the } \\
\text { DBS-off animal's } \\
\text { immobility. } \\
\text { Neither morphine nor } \\
\text { tramadol } \\
\text { counteracted the } \\
\text { antidepressant-like } \\
\text { effect of DBS-off } \\
\text { animals. }\end{array}$ \\
\hline
\end{tabular}


Table 3. Cont.

\begin{tabular}{|c|c|c|c|c|c|c|}
\hline Authors & Target & Animal & Animal Models \& DBS Design & Stimulation Parameters & Behavioral Tests & Outcomes \\
\hline & & & & & $\begin{array}{l}\text { Novelty suppressed } \\
\text { feeding test }\end{array}$ & $\begin{array}{c}\text { Electrode } \\
\text { implantation reduced } \\
\text { latency to feed } \\
\text { compared to naïve } \\
\text { animals. } \\
\text { Ibuprofen increased } \\
\text { latency to feed } \\
\text { relative to } \\
\text { VEH-treated animals. } \\
\text { Neither morphine nor } \\
\text { tramadol reduced the } \\
\text { latency to feed in } \\
\text { electrode-implanted } \\
\text { animals. }\end{array}$ \\
\hline $\begin{array}{l}\text { Torres-Sanchez et al. } \\
\text { (2018) [110] }\end{array}$ & vmPFC & $\begin{array}{l}\text { Wistar rats, } \\
\text { male. }\end{array}$ & $\begin{array}{l}\text { Naive animal model. } \\
\text { DBS for (i) } 4 \mathrm{~h} \text { at } 24 \mathrm{~h} \text { after surgery, then } \\
\text { (ii) } 2 \mathrm{~h} \text { at } 48 \mathrm{~h} \text { after surgery }\end{array}$ & $\begin{array}{l}\text { Bipolar Monophasic } \\
\text { Frequency: } 130 \mathrm{~Hz} \\
\text { Amplitude: } 100 \mu \mathrm{A} \\
\text { Pulse Width: } 90 \mu \mathrm{s}\end{array}$ & Forced swim test & $\begin{array}{c}\text { Reduced immobility } \\
\text { time and increased } \\
\text { climbing compared to } \\
\text { control. }\end{array}$ \\
\hline Volle et al. (2018) [119] & vmPFC & $\begin{array}{l}\text { Sprague-Dawley rats, } \\
\text { male. }\end{array}$ & $\begin{array}{l}\text { Stimulation was delivered } 1 \text { week after } \\
\text { surgery for either } \\
\text { (i) a single day (acute stimulation; } \\
8 \mathrm{~h} / \text { day) or } \\
\text { (ii) } 12 \text { days (chronic stimulation daily for } \\
8 \mathrm{~h} / \text { day) using a portable stimulator } \\
\text { (ANS model } 3510 \text { ) to different groups } \\
\text { of rats }\end{array}$ & $\begin{array}{l}\text { Frequency: } 130 \mathrm{~Hz} \\
\text { Amplitude: } 200 \mu \mathrm{A} \\
\text { Pulse Width: } 90 \mu \mathrm{s}\end{array}$ & N.A. & $\begin{array}{l}\text { Both treatments } \\
\text { increase serotonin } \\
\text { (5-HT) release, } \\
\text { although fluoxetine } \\
\text { resulted in a higher } \\
\text { sustained } \\
\text { concentration, even } \\
\text { upon chronic } \\
\text { treatment. Chronic } \\
\text { DBS resulted in } \\
\text { lowered 5HT release } \\
\text { by Day 12. DBS } \\
\text { reduced raphe SERT } \\
\text { expression. DBS } \\
\text { induced changes in } \\
\text { 5-HT1B receptor } \\
\text { expression, whereas } \\
\text { fluoxetine induced } \\
\text { changes in 5-HT1A } \\
\text { receptors expression } \\
\text { in the prefrontal } \\
\text { cortex. Research } \\
\text { highlighted different } \\
\text { effects of both } \\
\text { treatments on the } \\
\text { serotonergic system. }\end{array}$ \\
\hline
\end{tabular}


Table 3. Cont.

\begin{tabular}{|c|c|c|c|c|c|c|}
\hline Authors & Target & Animal & Animal Models \& DBS Design & Stimulation Parameters & Behavioral Tests & Outcomes \\
\hline \multirow{4}{*}{ Reznikov et al. (2017) [109] } & \multirow{4}{*}{$\mathrm{IL}$} & \multirow{4}{*}{$\begin{array}{l}\text { Sprague-Dawley rats, } \\
\text { male. }\end{array}$} & \multirow{4}{*}{$\begin{array}{l}\text { Posttraumatic stress disorder animal } \\
\text { model. } \\
\text { 3-day fear conditioning } \\
\text { DBS from } 1 \text { week after extinction recall } \\
\text { to the end of experiment, } 8 \mathrm{~h} / \text { day, or } 2 \mathrm{~h} \\
\text { before and } 4 \mathrm{~h} \text { after behavioral tests. }\end{array}$} & \multirow{4}{*}{$\begin{array}{l}\text { Frequency: } 130 \mathrm{~Hz} \\
\text { Amplitude: } 100 \mu \mathrm{A} \\
\text { Pulse Width: } 90 \mu \mathrm{s}\end{array}$} & Extinction recall test & $\begin{array}{l}\text { Higher freezing } \\
\text { scores in DBS-weak } \\
\text { extinction than } \\
\text { DBS-strong } \\
\text { extinction. }\end{array}$ \\
\hline & & & & & Open field test & $\begin{array}{c}\text { No significant } \\
\text { difference between } \\
\text { groups. }\end{array}$ \\
\hline & & & & & $\begin{array}{l}\text { Novelty suppressed } \\
\text { feeding test }\end{array}$ & $\begin{array}{l}\text { Reduced latency to } \\
\text { feeding in DBS-weak } \\
\text { extinction, but not } \\
\text { strong extinction. }\end{array}$ \\
\hline & & & & & Elevated plus maze & $\begin{array}{c}\text { No significant } \\
\text { difference observed } \\
\text { between groups. }\end{array}$ \\
\hline \multirow{3}{*}{$\begin{array}{l}\text { Bruchim-Samuel et al. } \\
\text { (2016) [96] }\end{array}$} & \multirow{3}{*}{$\begin{array}{l}\text { vmPFC } \\
\text { VTA }\end{array}$} & \multirow{3}{*}{$\begin{array}{c}\text { Flinders Sensitive Line rats, } \\
\text { male. } \\
\text { Sprague-Dawley rats, male. } \\
\text { (Control) }\end{array}$} & \multirow{3}{*}{$\begin{array}{l}\text { Flinders Sensitive Line model. } \\
\text { DBS for } 15 \mathrm{~min} / \text { day, for } 10 \text { days. }\end{array}$} & \multirow{3}{*}{$\begin{array}{c}\text { Bilateral, Monopolar } \\
\text { vmPFC Stimulation } \\
\text { Frequency: } 20 \mathrm{~Hz} \\
\text { Amplitude: } 400 \mu \mathrm{A} \\
\text { Pulse Width: } 200 \mu \mathrm{s} \\
\text { VTA Stimulation (control) } \\
\text { Frequency: } 10 \mathrm{~Hz} \\
\text { Amplitude: } 300 \mu \mathrm{A} \\
\text { Pulse Width: } 200 \mu \mathrm{s}\end{array}$} & $\begin{array}{l}\text { Sweetened condensed milk } \\
\text { intake test }\end{array}$ & $\begin{array}{c}\text { No significant } \\
\text { difference between } \\
\text { vmPFC groups. } \\
\text { Significant difference } \\
\text { between VTA groups } \\
\text { for Flinders Sensitive } \\
\text { Line rats. } \\
\end{array}$ \\
\hline & & & & & Novelty Exploration Test & $\begin{array}{c}\text { No significant } \\
\text { difference between } \\
\text { vmPFC groups. } \\
\text { Significant difference } \\
\text { between VTA groups } \\
\text { for Flinders sensitive } \\
\text { line rats. } \\
\end{array}$ \\
\hline & & & & & Forced swim test & $\begin{array}{c}\text { Decreased immobility } \\
\text { for } \\
\text { vmPFC-stimulated } \\
\text { rats after DBS for } 10 \\
\text { days, half relapsed at } \\
\text { day 28. } \\
\text { VTA-stimulated } \\
\text { Sprague-Dawley rats } \\
\text { had persistently } \\
\text { reduced immobility } \\
\text { until the end of the } \\
\text { experiment. }\end{array}$ \\
\hline $\begin{array}{c}\text { Jiménez-Sánchez et al. } \\
\text { (2016) [33] }\end{array}$ & $\mathrm{IL}$ & $\begin{array}{l}\text { Wistar rats, } \\
\text { male. }\end{array}$ & $\begin{array}{l}\text { Olfactory bulbectomized model animal } \\
\text { model. } \\
\text { DBS } 1 \mathrm{~h} \mathrm{daily} \mathrm{stimulation,} \mathrm{beginning} 2 \\
\text { days after electrode implantation before } \\
\text { behavioral testing. }\end{array}$ & $\begin{array}{l}\text { Bipolar Biphasic } \\
\text { Frequency: } 130 \mathrm{~Hz} \\
\text { Amplitude: } 200 \mu \mathrm{A} \\
\text { Pulse Width: } 90 \mu \mathrm{s}\end{array}$ & Social interaction test & $\begin{array}{l}\text { Increased duration of } \\
\text { active contact. }\end{array}$ \\
\hline
\end{tabular}


Table 3. Cont

\begin{tabular}{|c|c|c|c|c|c|c|}
\hline Authors & Target & Animal & Animal Models \& DBS Design & Stimulation Parameters & Behavioral Tests & Outcomes \\
\hline & & & & & Sucrose preference test & $\begin{array}{l}\text { Increased percentage } \\
\text { of sucrose } \\
\text { consumption in total } \\
\text { liquid consumption. }\end{array}$ \\
\hline & & & & & Forced swim test & $\begin{array}{l}\text { Reduced immobility } \\
\text { and increased } \\
\text { climbing but not } \\
\text { swimming. }\end{array}$ \\
\hline & & & & & Hyperemotionality test & $\begin{array}{c}\text { Reduced total } \\
\text { behavioral scores } \\
\text { when compared to } \\
\text { olfactory } \\
\text { bulbectomized sham } \\
\text { rats. } \\
\end{array}$ \\
\hline \multirow{3}{*}{$\begin{array}{l}\text { Jiménez-Sánchez et al. } \\
\text { (2016) [81] }\end{array}$} & \multirow{3}{*}{$\begin{array}{l}\mathrm{IL} \\
\mathrm{PrL}\end{array}$} & \multirow{3}{*}{$\begin{array}{l}\text { Wistar rats, } \\
\text { male. }\end{array}$} & \multirow{3}{*}{$\begin{array}{l}\text { Naïve animal model. } \\
\text { DBS for } 1 \mathrm{~h} \text { daily before behavioral } \\
\text { testing. }\end{array}$} & \multirow{3}{*}{$\begin{array}{l}\text { Bipolar Biphasic } \\
\text { Frequency: } 130 \mathrm{~Hz} \\
\text { Amplitude: } 200 \mu \mathrm{A} \\
\text { Pulse Width: } 90 \mu \mathrm{s}\end{array}$} & Forced swim test & $\begin{array}{c}\text { Reduced immobility } \\
\text { and increased } \\
\text { climbing in IL-DBS } \\
\text { No significant } \\
\text { behavioral changes in } \\
\text { PrL-DBS. } \\
\end{array}$ \\
\hline & & & & & Open field test & $\begin{array}{c}\text { Insignificant } \\
\text { locomotor changes in } \\
\text { IL-DBS. } \\
\end{array}$ \\
\hline & & & & & $\begin{array}{l}\text { Novelty suppressed } \\
\text { feeding test }\end{array}$ & $\begin{array}{l}\text { Decreased latency to } \\
\text { feed in IL-DBS. }\end{array}$ \\
\hline Rummel et al. (2016) [113] & vmPFC & $\begin{array}{c}\text { Flinders Sensitive Line rats, } \\
\text { male. } \\
\text { Congenitally learned } \\
\text { helplessness rats, } \\
\text { Male. }\end{array}$ & $\begin{array}{l}\text { Experiment } 1 \\
\text { Chronic intermittent DBS } 1 \text { week after } \\
\text { surgery in Flinders sensitive line rats, } 30 \\
\text { min each morning and extra } 30 \text {-min } \\
\text { stimulation on afternoons before the day } \\
\text { of behavioral test. } \\
\text { Experiment } 2 \\
\text { Chronic continuous DBS } 1 \text { week after } \\
\text { surgery for } 16 \text { days. } \\
\text { Experiment } 3 \\
\text { Chronic intermittent DBS in congenitally } \\
\text { learned helpless rats, procedures } \\
\text { followed that in experiment } 1 .\end{array}$ & $\begin{array}{c}\text { Chronic intermittent DBS } \\
\text { Frequency: } 130 \mathrm{~Hz} \\
\text { Amplitude: } 300 \mu \mathrm{A} \\
\text { Pulse Width: } 100 \mu \mathrm{s} \\
\text { Chronic continuous DBS } \\
\text { Frequency: } 130 \mathrm{~Hz} \\
\text { Amplitude: } 150 \mu \mathrm{A} \\
\text { Pulse Width: } 100 \mu \mathrm{s}\end{array}$ & Sucrose consumption test & $\begin{array}{l}\text { Chronic intermittent } \\
\text { DBS increased } \\
\text { sucrose intake in } \\
\text { Flinders sensitive line } \\
\text { rats but not in } \\
\text { congenitally learned } \\
\text { helplessness rats. } \\
\text { Chronic continuous } \\
\text { DBS did not affect } \\
\text { sucrose intake in } \\
\text { Flinders sensitive line } \\
\text { rats and } \\
\text { congenitally learned } \\
\text { helplessness rats. }\end{array}$ \\
\hline
\end{tabular}


Table 3. Cont.

\begin{tabular}{|c|c|c|c|c|c|c|}
\hline Authors & Target & Animal & Animal Models \& DBS Design & Stimulation Parameters & Behavioral Tests & Outcomes \\
\hline & & & & & Forced swim test & $\begin{array}{l}\text { Chronic intermittent } \\
\text { DBS and chronic } \\
\text { continuous DBS } \\
\text { increased latency to } \\
\text { immobility in } \\
\text { Flinders sensitive line } \\
\text { rats but not } \\
\text { congenitally learned } \\
\text { helplessness rats. }\end{array}$ \\
\hline & & & & & $\begin{array}{l}\text { Learned helplessness } \\
\text { paradigm }\end{array}$ & $\begin{array}{c}\text { Chronic intermittent } \\
\text { DBS and chronic } \\
\text { continuous DBS } \\
\text { decreased } \\
\text { helplessness in } \\
\text { Flinders sensitive line } \\
\text { rats but not cLH rats. }\end{array}$ \\
\hline & & & & & Sucrose intake test & $\begin{array}{c}\text { No significant } \\
\text { difference observed. }\end{array}$ \\
\hline & & & & & Novelty exploration test & $\begin{array}{c}\text { No significant } \\
\text { difference observed. }\end{array}$ \\
\hline Bambico et al. (2015) [98] & vmPFC & $\begin{array}{c}\text { Fisher rats, } \\
\text { Male. }\end{array}$ & $\begin{array}{c}\text { CUS animal model } \\
\text { CUS for } \sim 4 \text { weeks until anhedonia } \\
\text { inferred by SPI scores, then } \\
\text { performed implantation } \\
\text { DBS for } 3 \text { weeks after implantation, } 8 \mathrm{~h} \\
\text { per day, } 7 \text { days per week }\end{array}$ & $\begin{array}{l}\text { Frequency: } 130 \mathrm{~Hz} \\
\text { Amplitude: } 100 \mu \mathrm{A} \\
\text { Pulse Width: } 90 \mu \mathrm{s}\end{array}$ & $\begin{array}{l}\text { Novelty-suppressed } \\
\text { feeding test }\end{array}$ & $\begin{array}{l}\text { Reduced latency to } \\
\text { feeding in CUS-DBS } \\
\text { animals compared to } \\
\text { CUS-sham animals. }\end{array}$ \\
\hline
\end{tabular}


Table 3. Cont.

\begin{tabular}{|c|c|c|c|c|c|c|}
\hline Authors & Target & Animal & Animal Models \& DBS Design & Stimulation Parameters & Behavioral Tests & Outcomes \\
\hline & & & & & Open field test & $\begin{array}{c}\text { No significant } \\
\text { difference observed. }\end{array}$ \\
\hline & & & & & Elevated plus maze test & $\begin{array}{l}\text { More time in open } \\
\text { arms in CUS-DBS } \\
\text { animals compared to } \\
\text { CUS-sham animals. }\end{array}$ \\
\hline & & & & & Forced swim test & $\begin{array}{l}\text { Reduced immobility } \\
\text { time in CUS-DBS } \\
\text { animals compared to } \\
\text { CUS-sham animals. } \\
\end{array}$ \\
\hline $\begin{array}{l}\text { Edemann-Callesen et al. } \\
\text { (2015) [94] }\end{array}$ & $\begin{array}{l}\text { vmPFC; } \\
\text { Medial forebrain } \\
\text { bundle. }\end{array}$ & $\begin{array}{l}\text { Flinders sensitive line rats, } \\
\text { male. } \\
\text { Sprague-Dawley rats, male. }\end{array}$ & $\begin{array}{l}\text { Naïve animal model } \\
\text { DBS was applied in an Intra-cranial self- } \\
\text { stimulation protocol. }\end{array}$ & $\begin{array}{l}\text { Bilateral, Monopolar } \\
\text { Frequency: } 20-200 \mathrm{~Hz} \\
\text { Amplitude: } 170-560 \mu \mathrm{A} \\
\text { Pulse Width: } 100 \mu \mathrm{s}\end{array}$ & $\begin{array}{c}\text { Intra-cranial } \\
\text { self-stimulation }\end{array}$ & $\begin{array}{l}\text { For Flinders Sensitive } \\
\text { Line rats, } \\
\text { vmPFC-DBS did not } \\
\text { affect the } \\
\text { reward-seeking } \\
\text { behavior compared to } \\
\text { medial forebrain } \\
\text { bundle DBS. }\end{array}$ \\
\hline Etiévant et al. (2015) [103] & $\mathrm{IL}$ & Sprague-Dawley rats, male. & $\begin{array}{l}\text { Naïve animal model. } \\
\text { DBS for } 4 \mathrm{~h} \text { after forced swim pre-test } \\
\text { and } 2 \mathrm{~h} \text { before forced swim test. }\end{array}$ & $\begin{array}{l}\text { Bipolar Unilateral } \\
\text { Frequency: } 130 \mathrm{~Hz} \\
\text { Amplitude: } 150 \mu \mathrm{A} \\
\text { Pulse Width: } 60 \mu \mathrm{s}\end{array}$ & Forced swim test & $\begin{array}{l}\text { Reduced immobility } \\
\text { duration in IL-DBS } \\
\text { compared to control. }\end{array}$ \\
\hline Insel et al. (2015) [111] & $\mathrm{IL}$ & Sprague-Dawley rats, male. & $\begin{array}{l}\text { Naïve animal model. } \\
\text { DBS for } 8 \mathrm{~h} \text { per day, for } 10 \text { days. }\end{array}$ & $\begin{array}{l}\text { Monopolar Bilateral } \\
\text { Frequency: } 130 \mathrm{~Hz} \\
\text { Amplitude: } 100 \mu \mathrm{A} \\
\text { Pulse Width: } 90 \mu \mathrm{s}\end{array}$ & $\begin{array}{l}\text { Spontaneous behavior } \\
\text { recording }\end{array}$ & $\begin{array}{c}\text { Coherence between } \\
\text { ventral hippocampus } \\
\text { and IL was reduced } \\
\text { after 10-day DBS } \\
\text { compared to sham in } \\
2-4 \mathrm{~Hz} \text { brain activity } \\
\text { range, but was not } \\
\text { reduced after only } 1 \\
\text { day of treatment. } \\
\text { Coherence was not } \\
\text { affected by fluoxetine, } \\
\text { indicating that } \\
\text { IL-DBS observations } \\
\text { were independent of } \\
\text { the serotonergic } \\
\text { pathways. }\end{array}$ \\
\hline
\end{tabular}


Table 3. Cont.

\begin{tabular}{|c|c|c|c|c|c|c|}
\hline Authors & Target & Animal & Animal Models \& DBS Design & Stimulation Parameters & Behavioral Tests & Outcomes \\
\hline \multirow{5}{*}{ Lim et al. (2015) [102] } & \multirow{5}{*}{ vmPFC } & \multirow{5}{*}{ Sprague-Dawley rats, male. } & \multirow{5}{*}{$\begin{array}{c}\text { Experiment } 1 \\
\text { Naive animal model. } \\
\text { Experiment } 2 \\
\text { CUS animal model. } \\
\text { CUS for } 3 \text { weeks, each stressor lasted for } \\
10-14 \mathrm{~h} \\
\text { DBS for } 15 \text { min before home-cage } \\
\text { emergence test, before and during open } \\
\text { field test. }\end{array}$} & \multirow{5}{*}{$\begin{array}{l}\text { Bipolar Biphasic } \\
\text { Experiment } 1 \\
\text { Frequency:10/100 Hz } \\
\text { Amplitude: } 100 \mu \mathrm{A} \\
\text { Pulse Width: } 100 \mu \mathrm{s} \\
\text { Experiment } 2 \\
\text { Frequency: } 100 \mathrm{~Hz} \\
\text { Amplitude: } 100 \mu \mathrm{A} \\
\text { Pulse Width: } 100 \mu \mathrm{s}\end{array}$} & Home-cage emergence test & $\begin{array}{l}\text { HFS reduced escape } \\
\text { latency time in Naive } \\
\text { and CUS animal } \\
\text { model. }\end{array}$ \\
\hline & & & & & Open-field test & $\begin{array}{l}\text { Insignificant effect in } \\
\text { Naïve animals for } \\
\text { both HFS and LFS. } \\
\text { Increased time spent } \\
\text { in the central zone for } \\
\text { HFS-CUS. } \\
\end{array}$ \\
\hline & & & & & Food-intake test & $\begin{array}{l}\text { HFS increased food } \\
\text { intake in naïve } \\
\text { animals. } \\
\text { No significance } \\
\text { difference observed in } \\
\text { CUS animals. } \\
\end{array}$ \\
\hline & & & & & Sucrose-intake test & $\begin{array}{c}\text { Insignificant in Naïve } \\
\text { animals for both HFS } \\
\text { and LFS. } \\
\text { Increased sucrose } \\
\text { intake in HFS-CUS. }\end{array}$ \\
\hline & & & & & Forced swim test & $\begin{array}{l}\text { Insignificant in Naiive } \\
\text { for both HFS and LFS. } \\
\text { Reduced immobility } \\
\text { duration in HFS-CUS. }\end{array}$ \\
\hline \multirow[b]{2}{*}{ Lim et al. (2015) [108] } & \multirow[b]{2}{*}{ PrL } & \multirow[b]{2}{*}{ Sprague-Dawley rats, male. } & \multirow{2}{*}{$\begin{array}{l}\text { Naïve animal model. } \\
\text { DBS for } 15 \text { min before and during } \\
\text { sucrose intake test (same for forced } \\
\text { swim test) and before sacrifice for } 1 \mathrm{~h} \text {. }\end{array}$} & \multirow{2}{*}{$\begin{array}{l}\text { Bipolar Biphasic } \\
\text { Frequency: } 100 \mathrm{~Hz} \\
\text { Amplitude: } 100 \mu \mathrm{A} \\
\text { Pulse Width: } 100 \mu \mathrm{s}\end{array}$} & Sucrose intake test & $\begin{array}{l}\text { Increased sucrose } \\
\text { consumption. }\end{array}$ \\
\hline & & & & & Forced swim test & Reduced immobility. \\
\hline Liu et al. (2015) [99] & vmPFC & $\begin{array}{l}\text { Sprague-Dawley rats, male. } \\
4 \text { months old and } \\
12 \text { months old. }\end{array}$ & $\begin{array}{c}\text { Acute DBS } \\
\text { Naive animal model. } \\
\text { DBS for } 30 \text { min before the behavioral } \\
\text { tests. } \\
\text { Chronic DBS } \\
\text { Naive animal model. } \\
\text { DBS for } 1 \mathrm{~h} \text { daily including days of } \\
\text { behavioral tests. }\end{array}$ & $\begin{array}{c}\text { Bipolar Biphasic } \\
\text { Acute DBS } \\
\text { Frequency: } 10 / 100 \mathrm{~Hz} \\
\text { Amplitude: } 50 / 100 / 200 / 400 \mu \mathrm{A} \\
\text { Pulse Width: } 100 \mu \mathrm{s} \\
\text { Chronic DBS } \\
\text { Frequency: } 100 \mathrm{~Hz} \\
\text { Amplitude: } 100 \mu \mathrm{A} \\
\text { Pulse Width: } 100 \mu \mathrm{s}\end{array}$ & $\begin{array}{l}\text { Novel object } \\
\text { recognition test }\end{array}$ & $\begin{array}{l}\text { Acute HFS at } 200 \mu \mathrm{A} \\
\text { produced higher } \\
\text { novel object } \\
\text { exploration than } \\
\text { familiar object in } \\
\text { short-term } \\
\text { memory test. } \\
\text { Chronic HFS } \\
\text { increased novel object } \\
\text { exploration in short- } \\
\text { and long-term } \\
\text { memory than familiar } \\
\text { object, as well as the } \\
\text { durations. }\end{array}$ \\
\hline
\end{tabular}


Table 3. Cont.

\begin{tabular}{|c|c|c|c|c|c|c|}
\hline Authors & Target & Animal & Animal Models \& DBS Design & Stimulation Parameters & Behavioral Tests & Outcomes \\
\hline & & & & & Morris water maze & $\begin{array}{l}\text { Shorter latency to } \\
\text { reach platform on day } \\
1 \text { and } 2 \text { in chronic HFS } \\
\text { compared to sham. } \\
\text { More time spent in } \\
\text { the target quadrant } \\
\text { and less in the } \\
\text { opposite quadrant in } \\
\text { chronic HFS } \\
\text { compared to sham. }\end{array}$ \\
\hline \multirow[t]{2}{*}{ Hamani et al. (2014) [100] } & \multirow[t]{2}{*}{ vmPFC } & \multirow[t]{2}{*}{ Sprague-Dawley rats, male. } & \multirow{2}{*}{$\begin{array}{l}\text { Naïve animal model. } \\
\text { DBS for } 4 \mathrm{~h} \text { after FST on day } 1,2 \mathrm{~h} \text { before } \\
\text { swimming on day } 2 \\
\text { DBS } 1 \text { week after forced swim test, } 4 \mathrm{~h} \\
\text { on day } 1,2 \mathrm{~h} \text { on day } 2, \text { then assessed in } \\
\text { open field test }\end{array}$} & \multirow{2}{*}{$\begin{array}{l}\text { Monopolar } \\
\text { Frequency: } 130 \mathrm{~Hz} \\
\text { Amplitude: } 100 \mu \mathrm{A} \\
\text { Pulse Width: } 90 \mu \mathrm{s}\end{array}$} & Forced swim test & $\begin{array}{l}\text { Reduced immobility } \\
\text { and increased } \\
\text { climbing frequency } \\
\text { between groups. }\end{array}$ \\
\hline & & & & & Open field test & $\begin{array}{c}\text { No significant } \\
\text { difference in } \\
\text { locomotion observed. }\end{array}$ \\
\hline \multirow[t]{2}{*}{ Laver et al. (2014) [112] } & \multirow[t]{2}{*}{ vmPFC } & \multirow[t]{2}{*}{ Sprague-Dawley rats, male. } & \multirow[t]{2}{*}{$\begin{array}{l}\text { Naïve animal model. } \\
\text { Serotonin reuptake inhibitors/vehicle } \\
\text { were injected i.p. } 1 \mathrm{~h} \text { and } 5 \mathrm{~h} \text { after forced } \\
\text { swim test on day } 1 \text { and } 1 \mathrm{~h} \text { before forced } \\
\text { swim test on day } 2 \text {. } \\
\text { DBS for } 4 \mathrm{~h} \text { on day } 1 \text { of forced swim } \\
\text { pre-test and } 2 \mathrm{~h} \text { before forced swim test } \\
\text { on day } 2 \text {. }\end{array}$} & \multirow[t]{2}{*}{$\begin{array}{l}\text { Monopolar Bilateral } \\
\text { Frequency: } 130 \mathrm{~Hz} \\
\text { Amplitude: } 100 \mu \mathrm{A} \\
\text { Pulse Width: } 90 \mu \mathrm{s}\end{array}$} & Forced swim test & $\begin{array}{c}\text { DBS-saline, } \\
\text { DBS-buspirone, } \\
\text { DBS-Risperidone, } \\
\text { DBS-pindolol-treated } \\
\text { animals had higher } \\
\text { swimming and lower } \\
\text { observed immobility } \\
\text { frequencies. }\end{array}$ \\
\hline & & & & & Open field test & $\begin{array}{c}\text { No significant } \\
\text { difference observed }\end{array}$ \\
\hline $\begin{array}{l}\text { Perez-Caballero et al. (2013) } \\
\text { [45] }\end{array}$ & $\mathrm{IL}$ & $\begin{array}{l}\text { Wistar rats, } \\
\text { male. }\end{array}$ & $\begin{array}{l}\text { CUS animal model. } \\
\text { Electrode implantation after week } 4 \text { of } \\
\text { CUS, then CUS resumed. } \\
\text { DBS for } 4 \mathrm{~h} \text { after forced swim pre-test } \\
\text { and } 2 \mathrm{~h} \text { before forced swim test. } \\
\text { Some animals received pre-treatment } \\
\text { with indomethacin or ibuprofen. }\end{array}$ & $\begin{array}{l}\text { Bipolar Monophasic } \\
\text { Frequency: } 130 \mathrm{~Hz} \\
\text { Amplitude: } 100 \mu \mathrm{A} \\
\text { Pulse Width: } 90 \mu \mathrm{s}\end{array}$ & Forced swim test & $\begin{array}{c}\text { Reduced immobility } \\
\text { and increased } \\
\text { swimming in } \\
\text { DBS-off-IL and } \\
\text { DBS-on-IL compared } \\
\text { to sham- and } \\
\text { naïve-animals. } \\
\text { Increased immobility } \\
\text { and reduced } \\
\text { swimming in } \\
\text { DBS-off-IL animals } \\
\text { treated with NSAIDs. }\end{array}$ \\
\hline
\end{tabular}


Table 3. Cont

\begin{tabular}{|c|c|c|c|c|c|c|}
\hline Authors & Target & Animal & Animal Models \& DBS Design & Stimulation Parameters & Behavioral Tests & Outcomes \\
\hline & & & & & Open field test & $\begin{array}{l}\text { No significant } \\
\text { difference. }\end{array}$ \\
\hline \multirow{2}{*}{ Rea et al. (2014) [95] } & \multirow{2}{*}{ vmPFC } & \multirow{2}{*}{$\begin{array}{l}\text { Flinders sensitive line rats, } \\
\text { male. } \\
\text { Flinders resistant line rats, } \\
\text { male. } \\
\text { (Control) }\end{array}$} & \multirow{2}{*}{$\begin{array}{l}\text { DBS for } 30 \text { min each morning for } 2 \\
\text { weeks. } \\
\text { Extra DBS for } 30 \text { min in the afternoon } \\
\text { before the day of behavioral tests and } \\
\text { during behavioral tests. }\end{array}$} & \multirow{2}{*}{$\begin{array}{l}\text { Monopolar } \\
\text { Frequency: } 130 \mathrm{~Hz} \\
\text { Amplitude: } 300 \mu \mathrm{A} \\
\text { Pulse Width: } 100 \mu \mathrm{s}\end{array}$} & Forced swim test & $\begin{array}{c}\text { DBS reduced } \\
\text { immobility in both } \\
\text { groups of rats. }\end{array}$ \\
\hline & & & & & Sucrose consumption test & $\begin{array}{c}\text { DBS increased } \\
\text { sucrose consumption } \\
\text { in both groups of rats. }\end{array}$ \\
\hline $\begin{array}{l}\text { Veerakumar et al. } \\
\text { (2014) [104] }\end{array}$ & vmPFC & C57BL/6 mice, male. & $\begin{array}{c}\text { Chronic social defeat stress } \\
\text { animal model. } \\
\text { DBS for } 5 \mathrm{~h} / \text { day, for } 7 \text { days. }\end{array}$ & $\begin{array}{l}\text { Bipolar Unilateral } \\
\text { Frequency: } 160 \mathrm{~Hz} \\
\text { Amplitude: } 150 \mu \mathrm{A} \\
\text { Pulse Width: } 60 \mu \mathrm{s}\end{array}$ & Social interaction test & $\begin{array}{c}\text { Before DBS, } \\
\text { defeat-susceptible } \\
\text { mice showed lower } \\
\text { interaction times. } \\
\text { Defeated animals } \\
\text { with DBS spent } \\
\text { longer in the } \\
\text { interaction zone than } \\
\text { sham and similar to } \\
\text { non-stressed animals. } \\
\text { DBS increased the } \\
\text { total distance } \\
\text { traveled. }\end{array}$ \\
\hline Hamani et al. (2012) [92] & vmPFC & $\begin{array}{l}\text { Wistar rats, } \\
\text { male. }\end{array}$ & $\begin{array}{c}\text { CUS animal model. } \\
\text { DBS for } 8 \mathrm{~h} / \text { day, for } 2 \text { weeks }\end{array}$ & $\begin{array}{l}\text { Monopolar } \\
\text { Frequency: } 130 \mathrm{~Hz} \\
\text { Amplitude: } 200 \mu \mathrm{A} \\
\text { Pulse Width: } 90 \mu \mathrm{s}\end{array}$ & Sucrose preference test & $\begin{array}{l}\text { Higher preference } \\
\text { observed in } \\
\text { CUS-treated DBS } \\
\text { animals when } \\
\text { compared to } \\
\text { CUS-sham animals. } \\
\text { Higher sucrose } \\
\text { consumption in } \\
\text { CUS-treated DBS than } \\
\text { CUS-sham alone. }\end{array}$ \\
\hline Hamani et al. (2010) [93] & vmPFC, IL, PrL & $\begin{array}{c}\text { Sprague-Dawley rats, } \\
\text { male }\end{array}$ & $\begin{array}{l}\text { Naïve animal model } \\
\text { DBS for } 4 \mathrm{~h} \text { after FST on day } 1,2 \mathrm{~h} \text { before } \\
\text { swimming on day } 2\end{array}$ & $\begin{array}{c}\text { Frequency: } 20 \mathrm{~Hz} / 130 \mathrm{~Hz} \\
\text { Amplitude: } 100 / 200 / 300 / 400 \mu \mathrm{A} \\
\text { Pulse Width: } 90 \mu \mathrm{s}\end{array}$ & Forced swim test & $\begin{array}{c}\text { Parameters of } 130 \mathrm{~Hz}, \\
90 \mu \mathrm{s}, 200 \mu \mathrm{A} \text { reduced } \\
\text { immobility the most } \\
\text { in vmPFC-DBS, also } \\
\text { at } 100 \mu \mathrm{A} \text { and } 300 \mu \mathrm{A} \text {. } \\
\text { PrL stimulation at } 130 \\
\mathrm{~Hz}, 90 \mu \mathrm{s}, 200 \mu \mathrm{A} \\
\text { reduced immobility, } \\
\text { but IL stimulation } \\
\text { was insignificant. }\end{array}$ \\
\hline
\end{tabular}


Table 3. Cont.

\begin{tabular}{|c|c|c|c|c|c|c|}
\hline Authors & Target & Animal & Animal Models \& DBS Design & Stimulation Parameters & Behavioral Tests & Outcomes \\
\hline \multirow[t]{4}{*}{ Hamani et al. (2010) [91] } & \multirow[t]{4}{*}{ vmPFC } & \multirow[t]{4}{*}{$\begin{array}{l}\text { Sprague-Dawley rats, } \\
\text { male }\end{array}$} & \multirow[t]{4}{*}{$\begin{array}{l}\text { Naïve animal model, with serotonergic } \\
\text { depletion, or norepinephrine lesion. } \\
\text { DBS for Forced Swim Test } \\
\text { Day 1: } 4 \mathrm{~h} \text { after forced swim test } \\
\text { Day 2: } 2 \mathrm{~h} \text { before forced swim test } \\
\text { DBS for open field test, } \\
\text { novelty suppressed feeding test, } \\
\frac{\text { and learned helplessness. }}{\text { Pre-test Day 1: } 4 \mathrm{~h}} \\
\text { Pre-test Day 2: } 2 \mathrm{~h}\end{array}$} & \multirow[t]{4}{*}{$\begin{array}{l}\text { Monopolar } \\
\text { Frequency: } 130 \mathrm{~Hz} \\
\text { Amplitude: } 100 \mu \mathrm{A} \\
\text { Pulse Width: } 90 \mu \mathrm{s}\end{array}$} & Forced swim test & $\begin{array}{c}\text { DBS reduced } \\
\text { immobility and } \\
\text { increased swimming } \\
\text { counts in naïve } \\
\text { animals. } \\
\text { DBS in animals with } \\
\text { ibotenic acid injection } \\
\text { had lower immobility } \\
\text { and higher swimming } \\
\text { counts than control. } \\
\text { Rats with DBS } \\
\text { without serotonergic } \\
\text { depletion exhibited } \\
\text { lower immobility } \\
\text { than DBS animals } \\
\text { with serotonergic } \\
\text { depletion. } \\
\text { Rats with DBS } \\
\text { without } \\
\text { norepinephrine lesion } \\
\text { had lower immobility } \\
\text { than control, similar } \\
\text { reduction in } \\
\text { immobility was } \\
\text { shown in animals } \\
\text { with DBS and } \\
\text { norepinephrine } \\
\text { lesion. }\end{array}$ \\
\hline & & & & & Open field test & $\begin{array}{l}\text { No significant } \\
\text { difference. }\end{array}$ \\
\hline & & & & & $\begin{array}{l}\text { Novelty suppressed } \\
\text { feeding test }\end{array}$ & $\begin{array}{c}\text { DBS reduced latency } \\
\text { to feed compared to } \\
\text { control. }\end{array}$ \\
\hline & & & & & $\begin{array}{l}\text { Learned helplessness } \\
\text { paradigm }\end{array}$ & $\begin{array}{c}\text { Insignificant } \\
\text { difference in escape } \\
\text { latency between DBS } \\
\text { and control. }\end{array}$ \\
\hline
\end{tabular}


Table 3. Cont.

\begin{tabular}{|c|c|c|c|c|c|c|}
\hline Authors & Target & Animal & Animal Models \& DBS Design & Stimulation Parameters & Behavioral Tests & Outcomes \\
\hline & & & $\begin{array}{l}\text { Animals predisposed to helplessness. } \\
\text { DBS for } 2 \mathrm{~h} \text { before baseline assessment, } \\
2 \mathrm{~h} \text { before footshock at } 2 \text { days after } \\
\text { baseline test, and DBS for } 2 \mathrm{~h} \text { before } \\
\text { sucrose consumption test on the } \\
\text { next day }\end{array}$ & $\begin{array}{l}\text { Monopolar } \\
\text { Frequency: } 130 \mathrm{~Hz} \\
\text { Amplitude: } 100 \mu \mathrm{A} \\
\text { Pulse Width: } 90 \mu \mathrm{s}\end{array}$ & Sucrose consumption test & $\begin{array}{l}\text { DBS reduced the } \\
\text { sucrose drinking time } \\
\text { in animals after } \\
\text { footshock, but this } \\
\text { was insignificant. }\end{array}$ \\
\hline
\end{tabular}

Abbreviations: Cg, cingulate cortex; CUS, chronic unpredictable stress; EPM, elevated plus maze; FST, forced swim test; HFS, high-frequency stimulation; IL, infralimbic cortex; LFS, low-frequency stimulation; MWM, Morris water maze test; NORT, novel object recognition test; NSFT, novelty-suppressed feeding test; OBX, olfactory bulbectomy; OFT, open field test; PrL, prelimbic cortex; SD, Sprague-Dawley; SPI, sucrose preference index; vmPFC, ventromedial prefrontal cortex. 
Preclinical studies have progressed from studying the behavioral effects of DBS to understanding the accompanying cellular and molecular changes, be they local or distal nodes in the neurocircuitry. However, issues concerning the rodent homologs of SCC and the effect of stimulation in the subdivisions of vmPFC have yet to be resolved and are discussed in the later part of the review.

\section{Potential Mechanisms of Stimulation-Induced Antidepressant-Like Activities}

Several preclinical studies reported that DBS modulates neuronal activities in different brain regions, leading to antidepressant-like behaviors (Figure 1A). The network-wide cellular and molecular changes caused by vmPFC-DBS can be classified into neuroplasticity-dependent and -independent changes (Figure 1B). Neuroplasticity-dependent effects included neurogenesis, increased synaptic plasticity, enhanced neurotrophin signaling, and potential activation of glial cell-mediated changes, whereas neuroplasticity-independent effects included changes in serotonergic (5-HT) and glutamatergic neurotransmission patterns, either locally or in distal structures. Other changes outside the scope of this review might also be relevant.

(A)

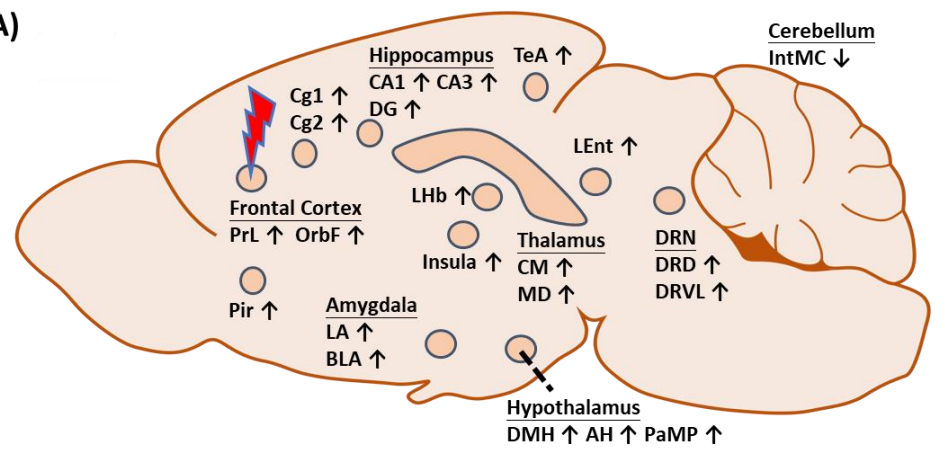

(B)

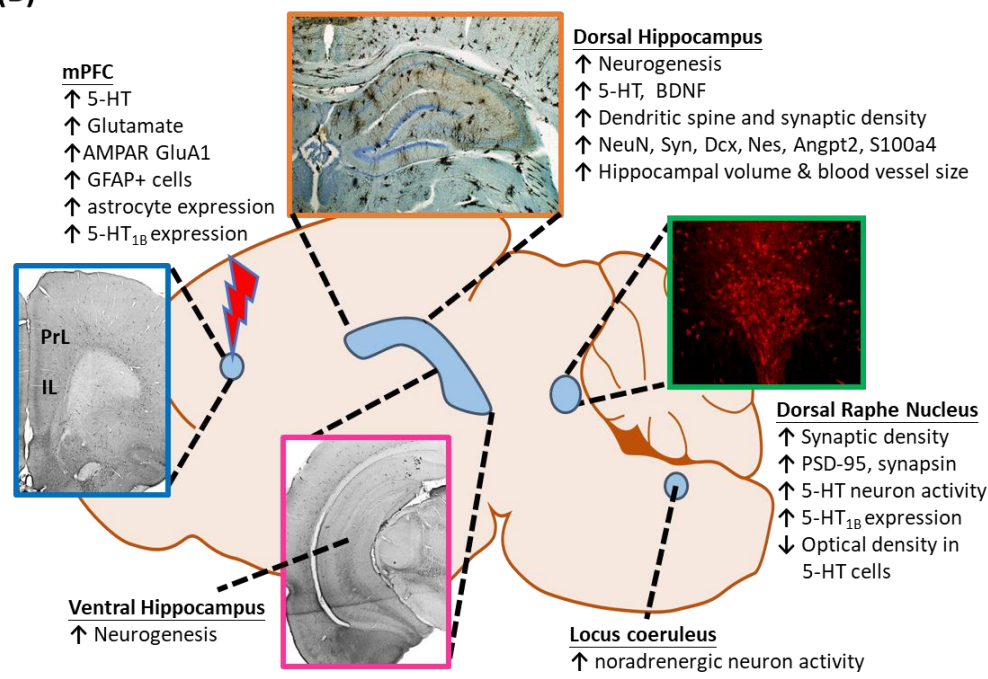

Figure 1. (A) Changes in local and distal neuronal activity after electrical stimulation of the ventromedial prefrontal cortex. (B) Neuroplasticity-dependent and -independent changes in different structures following vmPFC-DBS. Abbreviations: AH, anterior hypothalamus; AMPAR, $\alpha$-amino-3-hydroxy-5-methyl-4-isoxazolepropionic acid receptor; BDNF, brain-derived neurotrophic factor; BLA, basolateral amygdaloid nucleus; $\mathrm{Cg} 1,2$, cingulate gyrus area 1, 2; CM, centromedial thalamic nucleus; DG, dentate gyrus; DMH, dorsomedial hypothalamus; DRD, dorsal raphe nucleus, dorsal part; DRVL, dorsal raphe nucleus, ventrolateral part; IntMC, interposed cerebellar nucleus, magnocelluar part; LA, lateral amygdaloid nucleus; LEnt, lateral entorhinal cortex; LHb, lateral habenula; MD, mediodorsal thalamic nucleus; mPFC, medial prefrontal cortex; OrbF, orbitofrontal cortex; PaMP, paraventricular hypothalamic nucleus, medial parvicellular; Pir, piriform cortex; PrL, prelimbic cortex; TeA, temporal association area; and 5-HT, serotonin. 


\section{Neuroplasticity-Dependent Effects of Electrical Stimulation}

\section{(i) Neurogenesis is a Long-Term Cellular Change Brought About by Electrical Stimulation}

Post-mortem studies, pharmacological analyses, and electroconvulsive therapy reports have led to the neurogenic hypothesis of the pathogenesis of depression, whereby atrophy and apoptosis of hippocampal neurons correlated with depression and neurogenesis induce antidepressant-like effects [120,121]. As CA1 and subiculum in the hippocampus project substantially to the IL and the latter feeds back to the hippocampus via the relay nucleus reuniens in the thalamus [102,122], vmPFC-DBS induces a corollary of hippocampal neuromodulation that may mediate the antidepressant-like outcome. Etiévant et al. found that there was increased neurogenesis in the dentate gyrus of the dorsal and ventral hippocampus in rodents after 1-h IL-DBS, as detected by positive BrdU cells, and this was accompanied by reduced immobility in FST [103]. Similarly, Liu et al. reported proliferation of neuronal progenitors after chronic vmPFC-DBS, as demonstrated by increased positive BrdU and Dcx cell counts, as well as upregulated expressions of genes related to neurogenesis (NeuN, Syn, Dcx, Nes) and neuronal differentiation and protective functions (Angpt2, S100a4). These results were correlated with enhanced memory function, which may serve as another indication of vmPFC-DBS [99]. Bambico et al. confirmed that new cells with mature neuronal phenotype were found in the hippocampus after vmPFC-DBS, as detected by BrdU and NeuN co-expression. They also reported that temozolomide-induced reduction of these cells led to a longer latency to feed in a novelty-suppressed feeding test, but did not significantly change immobility in FST. This prompted the authors to further examine the anxiolytic-like and anti-anhedonic-like effects of vmPFC-DBS. In contrast, Winter et al. showed that $1 \mathrm{~h}$ of vmPFC-DBS with established DBS parameters in rodents did not increase the percentage of BrdU and Dcx double-stained cells in the dentate gyrus compared to the control [123]. Although research on the interaction between neurogenesis and substrates such as serotonin is ongoing, BDNF may be required to exert this antidepressant-like effect [98]. Neurogenesis is a widely investigated mechanism of DBS and these results indicate positive effects in the hippocampal region.

\section{(ii) Synaptic Plasticity is Altered More Rapidly by Electrical Stimulation than by Neurogenesis}

Disruption in synaptic functions and signaling are implicated in the pathophysiology of MDD, considering their importance in neurotransmission and ultimately, in cell survival [20]. Chronic stress, a risk factor of MDD, was shown to cause retraction of dendrites in the medial prefrontal cortex [124] and CA3 of the hippocampus [125] in rodents. In the latter, the long-term potentiation (LTP) of synapses was compromised, affecting long-term memory formation [126]. Regarding the changes in synaptic plasticity caused by vmPFC-DBS, Liu et al. reported denser secondary dendritic spines in the dentate gyrus, as demonstrated by upregulated Syn expression correlated with Nes and Dcx. The authors also reported a slight (1.2 fold) increase in hippocampal BDNF gene expression, a regulator of synaptic plasticity [99]. More recently, Bezchlibnyk et al. found that $1 \mathrm{~h}$ of IL-DBS resulted in longer dendritic length and branch points localized in the basal and apical regions of hippocampal CA1 neurons, respectively. These results indicated that the acute stimulation stressed the indispensable connections between the hippocampus and vmPFC, which may have implications in MDD and its treatment [127].

Chakravarty et al. found that 5 days of 6-h vmPFC-DBS daily in 9-week-old C57Bl/6 mice resulted in a larger hippocampal volume and increased hippocampal synaptic density, as indicated by upregulated synaptophysin expression, a presynaptic marker [128]. Similarly, Veerakumar et al. found that chronic vmPFC-DBS in transgenic mice increased dendrite length and complexity of the 5-HT DRN neurons and upregulated the expression of postsynaptic markers synaptophysin and PSD-95 [104]. Moreover, Etiévant et al. reported synaptogenesis in the DRN, as indicated by higher expressions of PSD-95 and synapsin. This may explain the prolonged DRN neuronal activation during and after vmPFC-DBS, leading to an antidepressant-like effect [103]. According to earlier reviews, dendritic spines can respond swiftly and provide surfaces for synapse formation [126,129]. Given the 
more dynamic properties of synapses compared to neurogenesis, synaptic plasticity may serve as an early indicator of vmPFC-DBS efficacy. More preclinical studies characterizing the dynamics of synaptic plasticity under vmPFC-DBS are anticipated.

\section{(iii) Neurotrophin Signaling Underlies the Antidepressant-Like Effect of Electrical Stimulation}

The neurotrophin BDNF is important in synaptic regulation, neuronal survival, and differentiation of new neuron terminals in the adult brain [130-132]. Preclinical studies reported that depressive-like rats subjected to chronic unpredictable stress [92,98] or olfactory bulbectomy [33] had lower BDNF levels, whereas DBS increased BDNF levels, thus preventing the development of depressive-like behaviors. Extracellularly, pro-BDNF is cleaved by tissue plasminogen activator/plasmin to form mature BDNF. The high-affinity tropomyosin-related kinase B (TrkB) receptor is activated by BDNF [133], leading to downstream phosphorylation of kinases, including protein kinase B (Akt) and extracellular signal-regulated kinases (ERK), which are important mediators of anti-apoptosis and proliferation, respectively [134]. Moreover, BDNF-TrkB triggers Serine 133 phosphorylation of transcription factor cAMP response element binding (CREB), leading to the formation of the dimer. The phosphorylated CREB dimer forms a larger transcriptional complex and alters multiple gene expressions including BDNF itself [135]. Encouragingly, Jiménez-Sánchez et al. showed that IL-DBS administered to olfactory bulbectomized rats activated these signaling pathways, as demonstrated by lowered Akt/pAkt, ERK/pERK, and CREB/pCREB ratios during $1 \mathrm{~h}$ of stimulation that increased again after stopping the stimulation, which was similar to the expression pattern of BDNF [33]. Further molecular studies are needed to characterize the action of vmPFC-DBS toward different targets in this signaling cascade.

\section{(iv) Potential Involvement of Glial Cells in Mediating the Outcome of Electrical Stimulation}

Glial cells may be involved in the pathogenesis of depression, as revealed by post-mortem studies of MDD patients, which found lower densities in the prefrontal cortex and amygdala, but increased levels in the hippocampal hilus [136-138]. The latter may be activated as a result of neuronal injury and decreasing neuronal populations $[139,140]$. Glial cells metabolically support neurons and regulate glutamate synthesis and thus, regulate synaptic plasticity. They may be modulated by DBS to potentiate the therapeutic effects [136]. This mechanism was supported in a study by Etiévant et al., which found that glial lesion by L-alpha-aminoadipic acid injection diminished antidepressant-like behaviors, hippocampal neurogenesis, and LTP induced by IL-DBS [103]. These findings led to the hypothesis that the neuronal-glial relationship is a determinant of the antidepressant-like efficacy of DBS, but this requires further study. Perez-Caballero et al. also studied the effects of electrode implantation and analgesic supplements [115]. They found that implantation with non-NSAID analgesic treatments, like tramadol and morphine, did not ameliorate the anti-depressant effects of the electrode implantation. This observation was accompanied by an increase in glial marker GFAP-positive cells. This finding suggests that the supplementation of non-NSAIDs postoperatively could improve the comfort of patients.

\section{Neuroplasticity-Independent Effects of Electrical Stimulation}

Besides modulating neuroplasticity-dependent mechanisms, DBS may manipulate some neuroplasticity-independent pathways to induce antidepressant-like effects. In a chronic mild stress model, depressive-like behaviors developed without significant deterioration of hippocampal neurogenesis or neuronal survival [141]. There are two likely inter-related neurotransmission systems that potentiate DBS efficacy, namely serotonergic and glutamatergic systems.

\section{(i) An Alternative Action of the Serotonergic System by Electrical Stimulation}

Results from preclinical studies have established an important role of the vmPFC-DRN axis and downstream 5-HT neurotransmission in the treatment of depression. Hamani et al. first reported that 5-HT neurotransmission was augmented by DBS, as shown by a four-fold increase in hippocampal 
5-HT after $1 \mathrm{~h}$ of vmPFC-DBS [91]. The authors also suggested a relationship between the integral 5-HT system and DBS efficacy, as 5-HT depletion induced by DRN lesions with 5,7-dihydroxytryptamine injection diminished the antidepressant-like effects of vmPFC-DBS [91,92]. Similarly, a study by Perez-Caballero et al. showed that the administration of para-chlorophenylalanine ester impeded 5-HT biosynthesis and diminished the antidepressant-like behaviors in early DBS among IL sham-treated animals [59]. Interestingly, Volle et al. showed that DBS and fluoxetine could rescue the 5-HT system via different mechanisms [119]. Both treatments increased the amount of 5-HT at the end of the chronic treatments, but chronic fluoxetine treatment was associated with decreased expression of 5- $\mathrm{HT}_{1 \mathrm{~A}}$ in the prefrontal cortex and the hippocampus, whereas chronic DBS increased 5- $\mathrm{HT}_{1 \mathrm{~B}}$ expression in the prefrontal cortex, globus pallidus, substantia nigra, and raphe nuclei.

A study by Veerakumar et al. in a transgenic mouse model of chronic social defeat stress revealed normalization of 5-HT neuron excitability in DRN after vmPFC-DBS [104]. Moreover, Jiménez-Sánchez et al. found increased prefrontal 5-HT efflux after $1 \mathrm{~h}$ of IL-DBS in olfactory bulbectomized rats [33] and in naive rats [81]. Etiévant et al. also found spontaneous DRN 5-HT neuron activity increased with IL-DBS [103]. Strikingly, an electrophysiological study performed by Srejic et al. showed that IL-DBS decreased the firing rate of DRN neurons, including serotonergic subtypes via the activation of GABAergic interneurons and possibly by the inhibition of excitatory glutamatergic neurons that modulate the firing of 5-HT [142]. Hence, the positive response to DBS could be enhanced by more selective targeting of the neuronal population by pharmacological adjuncts or coupling with optogenetic techniques. A study by Bregman et al. in 2018 using a serotonin transporter knockout mouse model found that DBS increased hippocampal 5-HT concentration, despite mice responding poorly to fluoxetine, a conventional selective serotonin reuptake inhibitor that acts on serotonin transporter [97]. These findings revealed a novel antidepressant-like activity of DBS involving the 5-HT system primarily in the DRN [79].

\section{(ii) Glutamatergic Neurotransmission is a Promising Target of Electrical Stimulation}

Jiménez-Sánchez et al. showed that there was enhanced prefrontal glutamatergic efflux together with changes in the local 5-HT profile $[33,81]$. The administration of AMPAR agonist and antagonist and subsequent FST showed that the increased glutamate led to antidepressant-like behaviors in animals [81]. The authors also found increased synthesis of the GluA1 subunit of AMPAR and postulated that their postsynaptic membrane insertion may explain the antidepressant-like outcome after $1 \mathrm{~h}$ of IL-DBS [33]. The activated glutamate output from the medial prefrontal cortex and frontal cortex enhanced 5-HT neuronal firing in the DRN [143], resulting in the antidepressant-like effect. However, Etiévant et al. argued that their activation was attributed to increased synaptogenesis in the DRN as previously described [103]. Nevertheless, Lim et al. hypothesized that a glutamatergic projection from the vmPFC to the medial subthalamic nucleus [144] may account for the antidepressant-like effects of vmPFC-DBS, as seen by increased c-Fos-immunoreactive cells in the medial subthalamic nucleus, increased sucrose consumption, and reduced immobility duration in FST [108]. With the emergence of glutamate-targeting pharmacotherapy [81], the ability to modulate glutamatergic transmission of DBS would add to the therapeutic novelty.

\section{Concerns and Limitations of the Electrical Stimulation Studies}

The small sample sizes in several clinical studies might compromise the credibility of the DBS efficacy, even in studies with similar recruited DBS subjects or consistent outcomes $[18,56]$. Most of the clinical studies were open-label, which means the responses could be prone to the placebo effect, despite the early response characterized by Perez-Caballero [59]. Efficacy of DBS treatment could be overestimated, unless countered by long stimulation, randomization, and blinding [18,145], such as double-blinded and sham-controlled studies [55]. A major criterion in preclinical studies is that they should mimic clinical studies. As it is unfeasible to stimulate animals for $24 \mathrm{~h}$ a day as in clinical designs [128], the scheduling of the stimulations and behavioral assessments will thus be relevant 
to the validity of the outcomes. Stimulation during behavioral tests will be most similar to clinical studies, but this may interfere with the physiological functions of the animals [79]. Besides, DBS is normally carried out in animals for relatively short periods and the effects might not correlate well with chronic stimulation [33]. Some preclinical studies were conducted in naive animal models and would not be compatible with clinical trials as TRD patients will be recruited exclusively in clinical settings $[33,93]$. Moreover, carry-over effects and lesion effects may interfere with the results in both settings. Carry-over effects refer to behavioral or neurochemical changes after DBS ceases. This needs to be counteracted by a washout period to allow the subjects to resume their baseline physiological states before the next stimulation [79]. Lesion effects occur where responses are observed after electrode implantation $[19,60,72]$. This needs to be differentiated from true responses observed in preclinical studies by sham-treatment [67], otherwise, the therapeutic effect will be over-estimated. Generally speaking, care must be taken in the design of experiments and data analysis of preclinical studies to increase their translational value to clinical studies.

\section{Prospective Approaches to Enhance Deep Brain Stimulation Safety and Efficacy}

Clinical response to SCC-DBS and various predictors can facilitate precise patient selection and customize the stimulation targets, thereby yielding maximal therapeutic outcomes with minimal adverse effects. For example, a lower baseline frontal theta cordance and incremental increase in the early stage of DBS indicates a clinical response [63]. Efforts have been made toward a more standardized approach to localize SCG in DBS responders [70]. Recently, real-time recording of the local field potential at the site of electrode implantation coupled with electroencephalogram have revealed network-wide clinical changes in DBS, which may improve the surgical precision [146]. Tractography-guided localization of electrodes, being more customized and precise, can improve the response rate [49]. A rechargeable DBS system should also be considered for long-term stimulation to reduce the need for surgery to replace batteries [17]. Lastly, given the high cost and invasiveness of DBS, more stringent regulation and evidence from randomized controlled studies are necessary to justify the benefits in TRD patients $[147,148]$.

\section{Conclusions}

Major depressive disorder is a debilitating psychiatric condition, which is affected by treatment resistance. Although safety concerns were raised on the risks of ablative treatment, it paved the way for deep brain stimulation as an adjustable therapy against depression. This review summarized the efficacies of deep brain stimulation in the subcallosal cingulate, one of the most extensively studied targets of stimulation, and in the ventromedial prefrontal cortex, which is the rodent homolog. Research on DBS initially focused on symptomatic relief. As the decades have progressed, studies have started to branch out and utilize modern technology to improve targeting of brain regions and to investigate a broader list of symptoms in patients. This has allowed us to better understand the impact of DBS on underreported parameters, such as heart rate, skin conductance, and brain waveforms. Additionally, preclinical research has expanded our understanding of the molecular factors modulated by stimulation. Besides the local effects, DBS has been shown to modulate distal structures, which can involve numerous projections to and from the stimulated targets, and can contribute to the antidepressant effects. This review also described some of the neuroplasticity-dependent and -independent changes brought about by DBS. Progress in different areas of research has helped lay the groundwork for the next wave of DBS research investigating more targeted and more effective applications of DBS for treating MDD. Last but not least, with further customization, more precise approaches, and more stringent regulation, it is anticipated that deep brain stimulation has great promise to treat severe, refractory depressive disorders in the near future.

Author Contributions: Methodology, Investigation, Formal Analysis, Data Curation, Visualization, \& Writing-Original Draft Preparation, Review \& Editing: S.K., F.Y.N. and L.W.L.; Conceptualization, Supervision, Project Administration, Resources, \& Funding Acquisition: L.W.L.; Validation: S.K., L.A., W.L.L. \& L.W.L.; 
Manuscript Intellectual Inputs: W.L.L., L.A., N.A.K., M.-L.F., Y.-S.C. and Y.T. All authors have read and agreed to the published version of the manuscript.

Funding: This scientific work was funded by grants from the Hong Kong Research Grants Council (27104616; 17119420) and the University of Hong Kong Seed Funding for Basic Research awarded to L.W.L.

Conflicts of Interest: The authors declare no conflict of interest.

\section{References}

1. Collaborators. GBD 2017 Disease and Injury Incidence and Prevalence. Global, regional, and national incidence, prevalence, and years lived with disability for 354 diseases and injuries for 195 countries and territories, 1990-2017: A systematic analysis for the Global Burden of Disease Study 2017. Lancet 2018, 392, 1789-1858.

2. Ferrari, A.J.; Charlson, F.J.; Norman, R.E.; Patten, S.B.; Freedman, G.; Murray, C.J.; Vos, T.; Whiteford, H.A. Burden of depressive disorders by country, sex, age, and year: Findings from the global burden of disease study 2010. PloS Med. 2013, 10, e1001547. [CrossRef] [PubMed]

3. Greenberg, P.E.; Fournier, A.-A.; Sisitsky, T.; Pike, C.T.; Kessler, R.C. The economic burden of adults with major depressive disorder in the United States (2005 and 2010). J. Clin. Psychiatry 2015, 76, 155-162. [CrossRef] [PubMed]

4. American Psychiatric Association. Diagnostic and Statistical Manual of Mental Disorders, 5th ed.; American Psychiatric Association Publishing: Washington, DC, USA, 2013.

5. Kendler, K.S.; Walters, E.E.; Neale, M.C.; Kessler, R.C.; Heath, A.C.; Eaves, L.J. The Structure of the Genetic and Environmental Risk Factors for Six Major Psychiatric Disorders in Women: Phobia, Generalized Anxiety Disorder, Panic Disorder, Bulimia, Major Depression, and Alcoholism. Arch. Gen. Psychiatry 1995, 52, $374-383$. [CrossRef] [PubMed]

6. Kendler, K.S.; Gardner, C.O.; Lichtenstein, P. A developmental twin study of symptoms of anxiety and depression: Evidence for genetic innovation and attenuation. Psychol. Med. 2008, 38, 1567-1575. [CrossRef]

7. Rudolph, M.; Rosanowski, F.; Eysholdt, U.; Kummer, P. Anxiety and depression in mothers of speech impaired children. Int. J. Pediatric Otorhinolaryngol. 2003, 67, 1337-1341. [CrossRef]

8. Montero-Pedrazuela, A.; Venero, C.; Lavado-Autric, R.; Fernández-Lamo, I.; García-Verdugo, J.M.; Bernal, J.; Guadaño-Ferraz, A. Modulation of adult hippocampal neurogenesis by thyroid hormones: Implications in depressive-like behavior. Mol. Psychiatry 2006, 11, 361-371. [CrossRef]

9. Monteagudo, P.T.; Falcão, A.A.; Verreschi, I.T.N.; Zanella, M.-T. The imbalance of sex-hormones related to depressive symptoms in obese men. Aging Male 2016, 19, 20-26. [CrossRef]

10. Rohr, U.D. The impact of testosterone imbalance on depression and women's health. Maturitas 2002, 41, 25-46. [CrossRef]

11. Malhi, G.S.; Bridges, P.K.; Malizia, A.L. Neurosurgery for mental disorders (NMD) A clinical worldwide perspective: Past, present and future. Int. J. Psychiatry Clin. Pract. 1997, 1, 119-129. [CrossRef]

12. López-Muñoz, F.; Alamo, C. Monoaminergic Neurotransmission: The History of the Discovery of Antidepressants from 1950s Until Today. Curr. Pharm. Des. 2009, 15, 1563-1586. [CrossRef]

13. Baghai, T.C.; Blier, P.; Baldwin, D.S.; Bauer, M.; Goodwin, G.M.; Fountoulakis, K.N.; Kasper, S.; Leonard, B.E.; Malt, U.F.; Stein, D.; et al. General and comparative efficacy and effectiveness of antidepressants in the acute treatment of depressive disorders: A report by the WPA section of pharmacopsychiatry. Eur. Arch. Psychiatry Clin. Neurosci. 2011, 261, 207-245. [CrossRef]

14. McInerney, S.J.; McNeely, H.E.; Geraci, J.; Giacobbe, P.; Rizvi, S.J.; Ceniti, A.K.; Cyriac, A.; Mayberg, H.S.; Lozano, A.M.; Kennedy, S.H. Neurocognitive Predictors of Response in Treatment Resistant Depression to Subcallosal Cingulate Gyrus Deep Brain Stimulation. Front. Hum. Neurosci. 2017, 11, 74. [CrossRef] [PubMed]

15. Holtzheimer, P.E.; Husain, M.M.; Lisanby, S.H.; Taylor, S.F.; Whitworth, L.A.; McClintock, S.; Slavin, K.V.; Berman, J.; McKhann, G.M.; Patil, P.G.; et al. Subcallosal cingulate deep brain stimulation for treatment-resistant depression: A multisite, randomised, sham-controlled trial. Lancet Psychiatry 2017, 4, 839-849. [CrossRef] 
16. Temel, Y.; Hescham, S.A.; Jahanshahi, A.; Janssen, M.L.; Tan, S.K.; van Overbeeke, J.J.; Ackermans, L.; Oosterloo, M.; Duits, A.; Leentjens, A.F.; et al. Neuromodulation in psychiatric disorders. Int. Rev. Neurobiol. 2012, 107, 283-314. [PubMed]

17. Morishita, T.; Fayad, S.M.; Higuchi, M.-a.; Nestor, K.A.; Foote, K.D. Deep Brain Stimulation for Treatment-resistant Depression: Systematic Review of Clinical Outcomes. Neurotherapeutics 2014, 11, 475-484. [CrossRef]

18. Kennedy, S.H.; Giacobbe, P.; Rizvi, S.J.; Placenza, F.M.; Nishikawa, Y.; Mayberg, H.S.; Lozano, A.M. Deep Brain Stimulation for Treatment-Resistant Depression: Follow-Up After 3 to 6 Years. Am. J. Psychiatry 2011, 168, 502-510. [CrossRef] [PubMed]

19. Mayberg, H.S.; Lozano, A.M.; Voon, V.; McNeely, H.E.; Seminowicz, D.; Hamani, C.; Schwalb, J.M.; Kennedy, S.H. Deep Brain Stimulation for Treatment-Resistant Depression. Neuron 2005, 45, 651-660. [CrossRef]

20. Duman, R.S.; Aghajanian, G.K.; Sanacora, G.; Krystal, J.H. Synaptic plasticity and depression: New insights from stress and rapid-acting antidepressants. Nat. Med. 2016, 22, 238-249. [CrossRef]

21. Gardner, J. A history of deep brain stimulation: Technological innovation and the role of clinical assessment tools. Soc. Stud. Sci. 2013, 43, 707-728. [CrossRef]

22. Benabid, A.L.; Pollak, P.; Louveau, A.; Henry, S.; de Rougemont, J. Combined (thalamotomy and stimulation) stereotactic surgery of the VIM thalamic nucleus for bilateral Parkinson disease. Appl. Neurophysiol. 1987, 50, 344-346. [CrossRef] [PubMed]

23. Faria, M.A. Violence, mental illness, and the brain-A brief history of psychosurgery: Part 2-From the limbic system and cingulotomy to deep brain stimulation. Surg. Neurol. Int. 2013, 4, 75. [CrossRef] [PubMed]

24. Krüger, S.; Seminowicz, D.; Goldapple, K.; Kennedy, S.H.; Mayberg, H.S. State and trait influences on mood regulation in bipolar disorder: Blood flow differences with an acute mood challenge. Biol. Psychiatry 2003, 54, 1274-1283. [CrossRef]

25. Zald, D.H.; Mattson, D.L.; Pardo, J.V. Brain activity in ventromedial prefrontal cortex correlates with individual differences in negative affect. Proc. Natl. Acad. Sci. USA 2002, 99, 2450-2454. [CrossRef]

26. Jimenez, F.; Velasco, F.; Salin-Pascual, R.; Hernandez, J.A.; Velasco, M.; Criales, J.L.; Nicolini, H. A patient with a resistant major depression disorder treated with deep brain stimulation in the inferior thalamic peduncle. Neurosurgery 2005, 57, 585-593. [CrossRef]

27. Schlaepfer, T.E.; Cohen, M.X.; Frick, C.; Kosel, M.; Brodesser, D.; Axmacher, N.; Joe, A.Y.; Kreft, M.; Lenartz, D.; Sturm, V. Deep brain stimulation to reward circuitry alleviates anhedonia in refractory major depression. Neuropsychopharmacology 2008, 33, 368-377. [CrossRef]

28. Schlaepfer, T.E.; Bewernick, B.H.; Kayser, S.; Madler, B.; Coenen, V.A. Rapid effects of deep brain stimulation for treatment-resistant major depression. Biol. Psychiatry 2013, 73, 1204-1212. [CrossRef]

29. Kringelbach, M.L.; Aziz, T.Z. Deep brain stimulation: Avoiding the errors of psychosurgery. JAMA 2009, 301, 1705-1707. [CrossRef]

30. Holtzheimer, P.E.; Mayberg, H.S. Neuromodulation for treatment-resistant depression. F1000 Med. Rep. 2012, 4, 22. [CrossRef]

31. Philip, M.L.; Richard, H.T.; Jeffrey, V.R.; Paul, B.F. Brain Neuromodulation Techniques: A Review. Neuroscientist 2016, 22, 406-421.

32. Blomstedt, P.; Hariz, M.I. Deep brain stimulation for movement disorders before DBS for movement disorders. Parkinsonism Relat. Disord. 2010, 16, 429-433. [CrossRef] [PubMed]

33. Jiménez-Sánchez, L.; Linge, R.; Campa, L.; Valdizán, E.M.; Pazos, Á.; Díaz, Á.; Adell, A. Behavioral, neurochemical and molecular changes after acute deep brain stimulation of the infralimbic prefrontal cortex. Neuropharmacology 2016, 108, 91-102. [CrossRef] [PubMed]

34. Greenberg, B.D.; Gabriels, L.A.; Malone, D.A.; Friehs, G.M.; Okun, M.S.; Shapira, N.A.; Foote, K.D.; Cosyns, P.R.; Kubu, C.S.; Malloy, P.F.; et al. Deep brain stimulation of the ventral internal capsule/ventral striatum for obsessive-compulsive disorder: Worldwide experience. Mol. Psychiatry 2010, 15, 64-79.

35. Kriston, L.; von Wolff, A. Not as golden as standards should be: Interpretation of the Hamilton Rating Scale for Depression. J. Affect. Disord. 2011, 128, 175-177. [CrossRef]

36. Hamani, C.; Mayberg, H.; Stone, S.; Laxton, A.; Haber, S.; Lozano, A.M. The subcallosal cingulate gyrus in the context of major depression. Biol. Psychiatry 2011, 69, 301-308. [CrossRef] 
37. Andrade, P.; Noblesse, L.H.M.; Temel, Y.; Ackermans, L.; Lim, L.W.; Steinbusch, H.W.M.; Visser-Vandewalle, V. Neurostimulatory and ablative treatment options in major depressive disorder: A systematic review. Acta Neurochir. 2010, 152, 565-577. [CrossRef]

38. Fitzgerald, P.B.; Laird, A.R.; Maller, J.; Daskalakis, Z.J. A meta-analytic study of changes in brain activation in depression. Hum. Brain Mapp. 2008, 29, 683-695. [CrossRef]

39. Mayberg, S.H.; Brannan, K.S.; Mahurin, K.R.; Jerabek, A.P.; Brickman, S.J.; Tekell, L.J.; Silva, A.J.; McGinnis, G.S.; Glass, C.T.; Martin, T.C.; et al. Cingulate function in depression: A potential predictor of treatment response. NeuroReport 1997, 8, 1057-1061. [CrossRef]

40. Sankar, T.; Chakravarty, M.M.; Jawa, N.; Li, S.X.; Giacobbe, P.; Kennedy, S.H.; Rizvi, S.J.; Mayberg, H.S.; Hamani, C.; Lozano, A.M. Neuroanatomical predictors of response to subcallosal cingulate deep brain stimulation for treatment-resistant depression. J. Psychiatry Neurosci. 2020, 45, 45-54. [CrossRef]

41. Riva-Posse, P.; Inman, C.S.; Choi, K.S.; Crowell, A.L.; Gross, R.E.; Hamann, S.; Mayberg, H.S. Autonomic arousal elicited by subcallosal cingulate stimulation is explained by white matter connectivity. Brain Stimul. 2019, 12, 743-751. [CrossRef]

42. Eitan, R.; Fontaine, D.; Benoit, M.; Giordana, C.; Darmon, N.; Israel, Z.; Linesky, E.; Arkadir, D.; Ben-Naim, S.; Iserlles, M.; et al. One year double blind study of high vs low frequency subcallosal cingulate stimulation for depression. J. Psychiatr. Res. 2018, 96, 124-134. [CrossRef]

43. Merkl, A.; Aust, S.; Schneider, G.-H.; Visser-Vandewalle, V.; Horn, A.; Kühn, A.A.; Kuhn, J.; Bajbouj, M. Deep brain stimulation of the subcallosal cingulate gyrus in patients with treatment-resistant depression: A double-blinded randomized controlled study and long-term follow-up in eight patients. J. Affect. Disord. 2018, 227, 521-529. [CrossRef]

44. Howell, B.; Choi, K.S.; Gunalan, K.; Rajendra, J.; Mayberg, H.S.; McIntyre, C.C. Quantifying the axonal pathways directly stimulated in therapeutic subcallosal cingulate deep brain stimulation. Hum. Brain Mapp. 2019, 40, 889-903. [CrossRef]

45. Waters, A.C.; Veerakumar, A.; Choi, K.S.; Howell, B.; Tiruvadi, V.; Bijanki, K.R.; Crowell, A.; Riva-Posse, P.; Mayberg, H.S. Test-retest reliability of a stimulation-locked evoked response to deep brain stimulation in subcallosal cingulate for treatment resistant depression. Hum. Brain Mapp. 2018, 39, 4844-4856. [CrossRef]

46. Smart, O.; Choi, K.S.; Riva-Posse, P.; Tiruvadi, V.; Rajendra, J.; Waters, A.C.; Crowell, A.L.; Edwards, J.; Gross, R.E.; Mayberg, H.S. Initial Unilateral Exposure to Deep Brain Stimulation in Treatment-Resistant Depression Patients Alters Spectral Power in the Subcallosal Cingulate. Front. Comput. Neurosci. $2018,12$. [CrossRef]

47. Choi, K.S.; Noecker, A.M.; Riva-Posse, P.; Rajendra, J.K.; Gross, R.E.; Mayberg, H.S.; McIntyre, C.C. Impact of brain shift on subcallosal cingulate deep brain stimulation. Brain Stimul. 2018, 11, 445-453. [CrossRef]

48. Conen, S.; Matthews, J.C.; Patel, N.K.; Anton-Rodriguez, J.; Talbot, P.S. Acute and chronic changes in brain activity with deep brain stimulation for refractory depression. J. Psychopharmacol. 2018, 32, 430-440. [CrossRef]

49. Riva-Posse, P.; Choi, K.S.; Holtzheimer, P.E.; Crowell, A.L.; Garlow, S.J.; Rajendra, J.K.; McIntyre, C.C.; Gross, R.E.; Mayberg, H.S. A connectomic approach for subcallosal cingulate deep brain stimulation surgery: Prospective targeting in treatment-resistant depression. Mol. Psychiatry 2017. [CrossRef]

50. Tsolaki, E.; Espinoza, R.; Pouratian, N. Using probabilistic tractography to target the subcallosal cingulate cortex in patients with treatment resistant depression. Psychiatry Res. Neuroimaging 2017, 261, 72-74. [CrossRef]

51. Accolla, E.A.; Aust, S.; Merkl, A.; Schneider, G.-H.; Kühn, A.A.; Bajbouj, M.; Draganski, B. Deep brain stimulation of the posterior gyrus rectus region for treatment resistant depression. J. Affect. Disord. 2016, 194, 33-37. [CrossRef]

52. Richieri, R.; Borius, P.Y.; Lagrange, G.; Faget-Agius, C.; Guedj, E.; Mc Gonigal, A.; Régis, J.M.; Lançon, C.; Bartolomei, F. Unmasking Partial Seizure after Deep Brain Stimulation for Treatment-Resistant Depression: A Case Report. Brain Stimul. 2016, 9, 636-638. [CrossRef]

53. Hilimire, M.R.; Mayberg, H.S.; Holtzheimer, P.E.; Broadway, J.M.; Parks, N.A.; DeVylder, J.E.; Corballis, P.M. Effects of Subcallosal Cingulate Deep Brain Stimulation on Negative Self-Bias in Patients with Treatment-Resistant Depression. Brain Stimul. 2015, 8, 185-191. [CrossRef] 
54. Martin-Blanco, A.; Serra-Blasco, M.; Perez-Egea, R.; de Diego-Adelino, J.; Carceller-Sindreu, M.; Puigdemont, D.; Molet, J.; Alvarez, E.; Perez, V.; Portella, M.J. Immediate cerebral metabolic changes induced by discontinuation of deep brain stimulation of subcallosal cingulate gyrus in treatment-resistant depression. J. Affect. Disord. 2015, 173, 159-162. [CrossRef]

55. Puigdemont, D.; Portella, M.J.; Pérez-Egea, R.; Molet, J.; Gironell, A.; de Diego-Adeliño, J.; Martín, A.; Rodríguez, R.; Àlvarez, E.; Artigas, F.; et al. A randomized double-blind crossover trial of deep brain stimulation of the subcallosal cingulate gyrus in patients with treatment-resistant depression: A pilot study of relapse prevention. J. Psychiatry Neurosci. Jpn. 2015, 40, 224-231. [CrossRef]

56. Serra-Blasco, M.; de Vita, S.; Rodriguez, M.R.; de Diego-Adelino, J.; Puigdemont, D.; Martin-Blanco, A.; Perez-Egea, R.; Molet, J.; Alvarez, E.; Perez, V.; et al. Cognitive functioning after deep brain stimulation in subcallosal cingulate gyrus for treatment-resistant depression: An exploratory study. Psychiatry Res. 2015, 225, 341-346. [CrossRef]

57. Choi, K.S.; Riva-Posse, P.; Gross, R.E.; Mayberg, H.S. Mapping the “Depression Switch” During Intraoperative Testing of Subcallosal Cingulate Deep Brain Stimulation. JAMA Neurol. 2015, 72, 1252-1260. [CrossRef]

58. Sun, Y.; Giacobbe, P.; Tang, C.W.; Barr, M.S.; Rajji, T.; Kennedy, S.H.; Fitzgerald, P.B.; Lozano, A.M.; Wong, W.; Daskalakis, Z.J. Deep Brain Stimulation Modulates Gamma Oscillations and Theta-Gamma Coupling in Treatment Resistant Depression. Brain Stimul. 2015, 8, 1033-1042. [CrossRef]

59. Perez-Caballero, L.; Pérez-Egea, R.; Romero-Grimaldi, C.; Puigdemont, D.; Molet, J.; Caso, J.R.; Mico, J.A.; Pérez, V.; Leza, J.C.; Berrocoso, E. Early responses to deep brain stimulation in depression are modulated by anti-inflammatory drugs. Mol. Psychiatry 2013, 19, 607. [CrossRef]

60. Merkl, A.; Schneider, G.-H.; Schönecker, T.; Aust, S.; Kühl, K.-P.; Kupsch, A.; Kühn, A.A.; Bajbouj, M. Antidepressant effects after short-term and chronic stimulation of the subgenual cingulate gyrus in treatment-resistant depression. Exp. Neurol. 2013, 249, 160-168. [CrossRef]

61. Ramasubbu, R.; Anderson, S.; Haffenden, A.; Chavda, S.; Kiss, Z.H. Double-blind optimization of subcallosal cingulate deep brain stimulation for treatment-resistant depression: A pilot study. J. Psychiatry Neurosci. 2013, 38, 325-332. [CrossRef] [PubMed]

62. Torres, C.V.; Ezquiaga, E.; Navas, M.; Sola, R.G. Deep brain stimulation of the subcallosal cingulate for medication-resistant type I bipolar depression: Case report. Bipolar. Disord. 2013, 15, 719-721. [CrossRef] [PubMed]

63. Broadway, J.M.; Holtzheimer, P.E.; Hilimire, M.R.; Parks, N.A.; DeVylder, J.E.; Mayberg, H.S.; Corballis, P.M. Frontal Theta Cordance Predicts 6-Month Antidepressant Response to Subcallosal Cingulate Deep Brain Stimulation for Treatment-Resistant Depression: A Pilot Study. Neuropsychopharmacology 2012, 37, 1764-1772. [CrossRef] [PubMed]

64. Hamani, C.; Giacobbe, P.; Diwan, M.; Balbino, E.S.; Tong, J.; Bridgman, A.; Lipsman, N.; Lozano, A.M.; Kennedy, S.H.; Nobrega, J.N. Monoamine oxidase inhibitors potentiate the effects of deep brain stimulation. Am. J. Psychiatry 2012, 169, 1320-1321. [CrossRef] [PubMed]

65. Holtzheimer, P.E.; Kelley, M.E.; Gross, R.E.; Filkowski, M.M.; Garlow, S.J.; Barrocas, A.; Wint, D.; Craighead, M.C.; Kozarsky, J.; Chismar, R.; et al. Subcallosal Cingulate Deep Brain Stimulation for Treatment-Resistant Unipolar and Bipolar Depression. Arch. Gen. Psychiatry 2012, 69, 150-158. [CrossRef] [PubMed]

66. Lozano, A.M.; Giacobbe, P.; Hamani, C.; Rizvi, S.J.; Kennedy, S.H.; Kolivakis, T.T.; Debonnel, G.; Sadikot, A.F.; Lam, R.W.; Howard, A.K.; et al. A multicenter pilot study of subcallosal cingulate area deep brain stimulation for treatment-resistant depression. J. Neurosurg. 2011, 116, 315-322. [CrossRef] [PubMed]

67. Puigdemont, D.; Pérez-Egea, R.; Portella, M.J.; Molet, J.; de Diego-Adeliño, J.; Gironell, A.; Radua, J.; Gómez-Anson, B.; Rodríguez, R.; Serra, M.; et al. Deep brain stimulation of the subcallosal cingulate gyrus: Further evidence in treatment-resistant major depression. Int. J. Neuropsychopharmacol. 2012, 15, 121-133. [CrossRef]

68. Guinjoan, S.M.; Mayberg, H.S.; Costanzo, E.Y.; Fahrer, R.D.; Tenca, E.; Antico, J.; Cerquetti, D.; Smyth, E.; Leiguarda, R.C.; Nemeroff, C.B. Asymmetrical contribution of brain structures to treatment-resistant depression as illustrated by effects of right subgenual cingulum stimulation. J. Neuropsychiatry Clin. Neurosci. 2010, 22, 265-277. [CrossRef] [PubMed]

69. Holtzheimer, P.E., 3rd; Mayberg, H.S. Deep brain stimulation for treatment-resistant depression. Am. J. Psychiatry 2010, 167, 1437-1444. [CrossRef] 
70. Hamani, C.; Mayberg, H.; Snyder, B.; Giacobbe, P.; Kennedy, S.; Lozano, A.M. Deep brain stimulation of the subcallosal cingulate gyrus for depression: Anatomical location of active contacts in clinical responders and a suggested guideline for targeting. J. Neurosurg. 2009, 111, 1209. [CrossRef]

71. Puigdemont, D.; Portella, M.J.; Pérez-Egea, R.; de Diego-Adeliño, J.; Gironell, A.; Molet, J.; Duran-Sindreu, S.; Álvarez, E.; Pérez, V. Depressive Relapse After Initial Response to Subcallosal Cingulate Gyrus-Deep Brain Stimulation in a Patient with a Treatment-Resistant Depression: Electroconvulsive Therapy as a Feasible Strategy. Biol. Psychiatry 2009, 66, e11-e12. [CrossRef]

72. Lozano, A.M.; Mayberg, H.S.; Giacobbe, P.; Hamani, C.; Craddock, R.C.; Kennedy, S.H. Subcallosal Cingulate Gyrus Deep Brain Stimulation for Treatment-Resistant Depression. Biol. Psychiatry 2008, 64, 461-467. [CrossRef] [PubMed]

73. McNeely, E.H.; Mayberg, S.H.; Lozano, M.A.; Kennedy, H.S. Neuropsychological Impact of Cg25 Deep Brain Stimulation for Treatment-Resistant Depression: Preliminary Results Over 12 Months. J. Nerv. Ment. Dis. 2008, 196, 405-410. [CrossRef] [PubMed]

74. Neimat, J.S.; Hamani, C.; Giacobbe, P.; Merskey, H.; Kennedy, S.H.; Mayberg, H.S.; Lozano, A.M. Neural stimulation successfully treats depression in patients with prior ablative cingulotomy. Am. J. Psychiatry 2008, 165, 687. [CrossRef] [PubMed]

75. Bari, A.A.; Mikell, C.B.; Abosch, A.; Ben-Haim, S.; Buchanan, R.J.; Burton, A.W.; Carcieri, S.; Cosgrove, G.R.; D'Haese, P.-F.; Daskalakis, Z.J.; et al. Charting the road forward in psychiatric neurosurgery: Proceedings of the 2016 American Society for Stereotactic and Functional Neurosurgery workshop on neuromodulation for psychiatric disorders. J. Neurol. Neurosurg. Psychiatry 2018, 89, 886-896. [CrossRef]

76. Moreines, J.L.; McClintock, S.M.; Kelley, M.E.; Holtzheimer, P.E.; Mayberg, H.S. Neuropsychological function before and after subcallosal cingulate deep brain stimulation in patients with treatment-resistant depression. Depress. Anxiety 2014, 31, 690-698. [CrossRef]

77. Nyandege, A.N.; Slattum, P.W.; Harpe, S.E. Risk of Fracture and the Concomitant Use of Bisphosphonates With Osteoporosis-Inducing Medications. Ann. Pharmacother. 2015, 49, 437-447. [CrossRef]

78. Cox, G.R.; Callahan, P.; Churchill, R.; Hunot, V.; Merry, S.N.; Parker, A.G.; Hetrick, S.E. Psychological therapies versus antidepressant medication, alone and in combination for depression in children and adolescents. Cochrane Database Syst. Rev. 2014, 11, CD008324. [CrossRef]

79. Hamani, C.; Nobrega, J.N. Preclinical Studies Modeling Deep Brain Stimulation for Depression. Biol. Psychiatry 2012, 72, 916-923. [CrossRef]

80. Vertes, R.P. Interactions among the medial prefrontal cortex, hippocampus and midline thalamus in emotional and cognitive processing in the rat. Neuroscience 2006, 142, 1-20. [CrossRef]

81. Jiménez-Sánchez, L.; Castañé, A.; Pérez-Caballero, L.; Grifoll-Escoda, M.; López-Gil, X.; Campa, L.; Galofré, M.; Berrocoso, E.; Adell, A. Activation of AMPA Receptors Mediates the Antidepressant Action of Deep Brain Stimulation of the Infralimbic Prefrontal Cortex. Cereb. Cortex 2016, 26, 2778-2789. [CrossRef]

82. Ferry, A.T.; Öngür, D.; An, X.; Price, J.L. Prefrontal cortical projections to the striatum in macaque monkeys: Evidence for an organization related to prefrontal networks. J. Comp. Neurol. 2000, 425, 447-470. [CrossRef]

83. McFarland, N.R.; Haber, S.N. Thalamic Relay Nuclei of the Basal Ganglia Form Both Reciprocal and Nonreciprocal Cortical Connections, Linking Multiple Frontal Cortical Areas. J. Neurosci. 2002, 22, 8117-8132. [CrossRef]

84. Parthoens, J.; Verhaeghe, J.; Stroobants, S.; Staelens, S. Deep Brain Stimulation of the Prelimbic Medial Prefrontal Cortex: Quantification of the Effect on Glucose Metabolism in the Rat Brain Using [18 F] FDG MicroPET. Mol. Imaging Biol. 2014, 16, 838-845. [CrossRef]

85. Hajós, M.; Richards, C.D.; Székely, A.D.; Sharp, T. An electrophysiological and neuroanatomical study of the medial prefrontal cortical projection to the midbrain raphe nuclei in the rat. Neuroscience 1998, 87, 95-108. [CrossRef]

86. Wood, M.; Adil, O.; Wallace, T.; Fourman, S.; Wilson, S.P.; Herman, J.P.; Myers, B. Infralimbic prefrontal cortex structural and functional connectivity with the limbic forebrain: A combined viral genetic and optogenetic analysis. Brain Struct. Funct. 2019, 224, 73-97. [CrossRef] [PubMed]

87. Sesack, S.R.; Deutch, A.Y.; Roth, R.H.; Bunney, B.S. Topographical organization of the efferent projections of the medial prefrontal cortex in the rat: An anterograde tract-tracing study with Phaseolus vulgaris leucoagglutinin. J. Comp. Neurol. 1989, 290, 213-242. [CrossRef] [PubMed] 
88. Chandler, L.J.; Gass, J.T. The plasticity of extinction: Contribution of the prefrontal cortex in treating addiction though inhibitory learning. Front. Psychiatry 2013, 4. [CrossRef]

89. Uylings, H.B.; van Eden, C.G. Qualitative and quantitative comparison of the prefrontal cortex in rat and in primates, including humans. Prog. Brain Res. 1990, 85, 31-62. [PubMed]

90. Lim, L.W.; Tan, S.K.; Groenewegen, H.J.; Temel, Y. Electrical brain stimulation in depression: Which target(s)? Biol. Psychiatry 2011, 69, e5-e6, author reply e7-e8. [CrossRef] [PubMed]

91. Hamani, C.; Diwan, M.; Macedo, C.E.; Brandao, M.L.; Shumake, J.; Gonzalez-Lima, F.; Raymond, R.; Lozano, A.M.; Fletcher, P.J.; Nobrega, J.N. Antidepressant-like effects of medial prefrontal cortex deep brain stimulation in rats. Biol. Psychiatry 2010, 67, 117-124. [CrossRef]

92. Hamani, C.; Machado, D.C.; Hipólide, D.C.; Dubiela, F.P.; Suchecki, D.; Macedo, C.E.; Tescarollo, F.; Martins, U.; Covolan, L.; Nobrega, J.N. Deep Brain Stimulation Reverses Anhedonic-Like Behavior in a Chronic Model of Depression: Role of Serotonin and Brain Derived Neurotrophic Factor. Biol. Psychiatry 2012, 71, 30-35. [CrossRef] [PubMed]

93. Hamani, C.; Diwan, M.; Isabella, S.; Lozano, A.M.; Nobrega, J.N. Effects of different stimulation parameters on the antidepressant-like response of medial prefrontal cortex deep brain stimulation in rats. J. Psychiatr. Res. 2010, 44, 683-687. [CrossRef] [PubMed]

94. Edemann-Callesen, H.; Voget, M.; Empl, L.; Vogel, M.; Wieske, F.; Rummel, J.; Heinz, A.; Mathé, A.A.; Hadar, R.; Winter, C. Medial Forebrain Bundle Deep Brain Stimulation has Symptom-specific Anti-depressant Effects in Rats and as Opposed to Ventromedial Prefrontal Cortex Stimulation Interacts With the Reward System. Brain Stimul. 2015, 8, 714-723. [CrossRef] [PubMed]

95. Rea, E.; Rummel, J.; Schmidt, T.T.; Hadar, R.; Heinz, A.; Mathé, A.A.; Winter, C. Anti-Anhedonic Effect of Deep Brain Stimulation of the Prefrontal Cortex and the Dopaminergic Reward System in a Genetic Rat Model of Depression: An Intracranial Self-Stimulation Paradigm Study. Brain Stimul. 2014, 7, 21-28. [CrossRef] [PubMed]

96. Bruchim-Samuel, M.; Lax, E.; Gazit, T.; Friedman, A.; Ahdoot, H.; Bairachnaya, M.; Pinhasov, A.; Yadid, G. Electrical stimulation of the vmPFC serves as a remote control to affect VTA activity and improve depressive-like behavior. Exp. Neurol. 2016, 283, 255-263. [CrossRef] [PubMed]

97. Bregman, T.; Nona, C.; Volle, J.; Diwan, M.; Raymond, R.; Fletcher, P.J.; Nobrega, J.N.; Hamani, C. Deep brain stimulation induces antidepressant-like effects in serotonin transporter knockout mice. Brain Stimul. 2018, 11, 423-425. [CrossRef]

98. Bambico, F.R.; Bregman, T.; Diwan, M.; Li, J.; Darvish-Ghane, S.; Li, Z.; Laver, B.; Amorim, B.O.; Covolan, L.; Nobrega, J.N.; et al. Neuroplasticity-dependent and -independent mechanisms of chronic deep brain stimulation in stressed rats. Transl. Psychiatry 2015, 5, e674. [CrossRef]

99. Liu, A.; Jain, N.; Vyas, A.; Lim, L.W. Ventromedial prefrontal cortex stimulation enhances memory and hippocampal neurogenesis in the middle-aged rats. eLife 2015, 4, e04803. [CrossRef]

100. Hamani, C.; Amorim, B.O.; Wheeler, A.L.; Diwan, M.; Driesslein, K.; Covolan, L.; Butson, C.R.; Nobrega, J.N. Deep brain stimulation in rats: Different targets induce similar antidepressant-like effects but influence different circuits. Neurobiol. Dis. 2014, 71, 205-214. [CrossRef]

101. Bregman, T.; Reznikov, R.; Diwan, M.; Raymond, R.; Butson, C.R.; Nobrega, J.N.; Hamani, C. Antidepressant-like Effects of Medial Forebrain Bundle Deep Brain Stimulation in Rats are not Associated With Accumbens Dopamine Release. Brain Stimul. 2015, 8, 708-713. [CrossRef]

102. Lim, L.W.; Prickaerts, J.; Huguet, G.; Kadar, E.; Hartung, H.; Sharp, T.; Temel, Y. Electrical stimulation alleviates depressive-like behaviors of rats: Investigation of brain targets and potential mechanisms. Transl. Psychiatry 2015, 5, e535. [CrossRef] [PubMed]

103. Etiévant, A.; Oosterhof, C.; Bétry, C.; Abrial, E.; Novo-Perez, M.; Rovera, R.; Scarna, H.; Devader, C.; Mazella, J.; Wegener, G.; et al. Astroglial Control of the Antidepressant-Like Effects of Prefrontal Cortex Deep Brain Stimulation. EBioMedicine 2015, 2, 898-908. [CrossRef] [PubMed]

104. Veerakumar, A.; Challis, C.; Gupta, P.; Da, J.; Upadhyay, A.; Beck, S.G.; Berton, O. Antidepressant-like effects of cortical deep brain stimulation coincide with pro-neuroplastic adaptations of serotonin systems. Biol. Psychiatry 2014, 76, 203-212. [CrossRef] [PubMed]

105. Bhaskar, Y.; Lim, L.W.; Mitra, R. Enriched Environment Facilitates Anxiolytic Efficacy Driven by Deep-Brain Stimulation of Medial Prefrontal Cortex. Front. Behav. Neurosci. 2018, 12, 204. [CrossRef] [PubMed] 
106. Creed, M.C.; Hamani, C.; Nobrega, J.N. Effects of repeated deep brain stimulation on depressive- and anxiety-like behavior in rats: Comparing entopeduncular and subthalamic nuclei. Brain Stimul. 2013, 6, 506-514. [CrossRef]

107. Meng, H.; Wang, Y.; Huang, M.; Lin, W.; Wang, S.; Zhang, B. Chronic deep brain stimulation of the lateral habenula nucleus in a rat model of depression. Brain Res. 2011, 1422, 32-38. [CrossRef]

108. Lim, L.W.; Janssen, M.L.F.; Kocabicak, E.; Temel, Y. The antidepressant effects of ventromedial prefrontal cortex stimulation is associated with neural activation in the medial part of the subthalamic nucleus. Behav. Brain Res. 2015, 279, 17-21. [CrossRef]

109. Reznikov, R.; Bambico, F.R.; Diwan, M.; Raymond, R.J.; Nashed, M.G.; Nobrega, J.N.; Hamani, C. Prefrontal Cortex Deep Brain Stimulation Improves Fear and Anxiety-Like Behavior and Reduces Basolateral Amygdala Activity in a Preclinical Model of Posttraumatic Stress Disorder. Neuropsychopharmacology 2017, 43, 1099-1106. [CrossRef]

110. Torres-Sanchez, S.; Perez-Caballero, L.; Mico, J.A.; Celada, P.; Berrocoso, E. Effect of Deep Brain Stimulation of the ventromedial prefrontal cortex on the noradrenergic system in rats. Brain Stimul. 2018, 11, 222-230. [CrossRef]

111. Insel, N.; Pilkiw, M.; Nobrega, J.N.; Hutchison, W.D.; Takehara-Nishiuchi, K.; Hamani, C. Chronic deep brain stimulation of the rat ventral medial prefrontal cortex disrupts hippocampal-prefrontal coherence. Exp. Neurol. 2015, 269, 1-7. [CrossRef]

112. Laver, B.; Diwan, M.; Nobrega, J.N.; Hamani, C. Augmentative therapies do not potentiate the antidepressant-like effects of deep brain stimulation in rats. J. Affect. Disord. 2014, 161, 87-90. [CrossRef]

113. Rummel, J.; Voget, M.; Hadar, R.; Ewing, S.; Sohr, R.; Klein, J.; Sartorius, A.; Heinz, A.; Mathé, A.A.; Vollmayr, B.; et al. Testing different paradigms to optimize antidepressant deep brain stimulation in different rat models of depression. J. Psychiatr. Res. 2016, 81, 36-45. [CrossRef] [PubMed]

114. Lehto, L.J.; Filip, P.; Laakso, H.; Sierra, A.; Slopsema, J.P.; Johnson, M.D.; Eberly, L.E.; Low, W.C.; Grohn, O.; Tanila, H.; et al. Tuning Neuromodulation Effects by Orientation Selective Deep Brain Stimulation in the Rat Medial Frontal Cortex. Front. Neurosci. 2018, 12, 899. [CrossRef] [PubMed]

115. Perez-Caballero, L.; Soto-Montenegro, M.L.; Hidalgo-Figueroa, M.; Mico, J.A.; Desco, M.; Berrocoso, E. Deep brain stimulation electrode insertion and depression: Patterns of activity and modulation by analgesics. Brain Stimul. 2018, 11, 1348-1355. [CrossRef] [PubMed]

116. Jia, L.; Sun, Z.; Shi, D.; Wang, M.; Jia, J.; He, Y.; Xue, F.; Ren, Y.; Yang, J.; Ma, X. Effects of different patterns of electric stimulation of the ventromedial prefrontal cortex on hippocampal-prefrontal coherence in a rat model of depression. Behav. Brain Res. 2019, 356, 179-188. [CrossRef]

117. Papp, M.; Gruca, P.; Lason, M.; Niemczyk, M.; Willner, P. The role of prefrontal cortex dopamine D2 and D3 receptors in the mechanism of action of venlafaxine and deep brain stimulation in animal models of treatment-responsive and treatment-resistant depression. J. Psychopharmacol. 2019, 33, 748-756. [CrossRef]

118. Papp, M.; Gruca, P.; Lason, M.; Tota-Glowczyk, K.; Niemczyk, M.; Litwa, E.; Willner, P. Rapid antidepressant effects of deep brain stimulation of the pre-frontal cortex in an animal model of treatment-resistant depression. J. Psychopharmacol 2018, 32, 1133-1140. [CrossRef]

119. Volle, J.; Bregman, T.; Scott, B.; Diwan, M.; Raymond, R.; Fletcher, P.J.; Nobrega, J.N.; Hamani, C. Deep brain stimulation and fluoxetine exert different long-term changes in the serotonergic system. Neuropharmacology 2018, 135, 63-72. [CrossRef]

120. Santarelli, L.; Saxe, M.; Gross, C.; Surget, A.; Battaglia, F.; Dulawa, S.; Weisstaub, N.; Lee, J.; Duman, R.; Arancio, O.; et al. Requirement of Hippocampal Neurogenesis for the Behavioral Effects of Antidepressants. Science 2003, 301, 805. [CrossRef]

121. Pittenger, C.; Duman, R.S. Stress, depression, and neuroplasticity: A convergence of mechanisms. Neuropsychopharmacology 2008, 33, 88-109. [CrossRef]

122. Vertes, R.P.; Hoover, W.B.; Szigeti-Buck, K.; Leranth, C. Nucleus reuniens of the midline thalamus: Link between the medial prefrontal cortex and the hippocampus. Brain Res. Bull. 2007, 71, 601-609. [CrossRef]

123. Winter, C.; Bregman, T.; Voget, M.; Raymond, R.; Hadar, R.; Nobrega, J.N.; Hamani, C. Acute high frequency stimulation of the prefrontal cortex or nucleus accumbens does not increase hippocampal neurogenesis in rats. J. Psychiatr. Res. 2015, 68, 27-29. [CrossRef] [PubMed] 
124. Cook, S.C.; Wellman, C.L. Chronic stress alters dendritic morphology in rat medial prefrontal cortex. J. Neurobiol. 2004, 60, 236-248. [CrossRef] [PubMed]

125. Magariños, A.M.; McEwen, B.S.; Flügge, G.; Fuchs, E. Chronic psychosocial stress causes apical dendritic atrophy of hippocampal CA3 pyramidal neurons in subordinate tree shrews. J. Neurosci. Off. J. Soc. Neurosci. 1996, 16, 3534. [CrossRef]

126. Shepherd, G.M. The dendritic spine: A multifunctional integrative unit. J. Neurophysiol. 1996, 75, $2197-2210$. [CrossRef] [PubMed]

127. Bezchlibnyk, Y.B.; Stone, S.S.D.; Hamani, C.; Lozano, A.M. High frequency stimulation of the infralimbic cortex induces morphological changes in rat hippocampal neurons. Brain Stimul. 2017, 10, 315-323. [CrossRef] [PubMed]

128. Chakravarty, M.M.; Hamani, C.; Martinez-Canabal, A.; Ellegood, J.; Laliberté, C.; Nobrega, J.N.; Sankar, T.; Lozano, A.M.; Frankland, P.W.; Lerch, J.P. Deep brain stimulation of the ventromedial prefrontal cortex causes reorganization of neuronal processes and vasculature. NeuroImage 2016, 125, 422-427. [CrossRef] [PubMed]

129. Hering, H.; Sheng, M. Dendritic spines: Structure, dynamics and regulation. Nat. Rev. Neurosci. 2001, 2, 880. [CrossRef]

130. Kuem-Ju, L.; Sung-Jin, K.; Suk-Won, K.; Song-Hyen, C.; You-Chan, S.; Sang-Ha, P.; Bo-Hyun, M.; Eujin, C.; Min-Soo, L.; Sang-Hyun, C.; et al. Chronic mild stress decreases survival, but not proliferation, of new-born cells in adult rat hippocampus. Exp. Amp; Mol. Med. 2006, 38, 44.

131. Gulyaeva, N.V. Molecular Mechanisms of Neuroplasticity: An Expanding Universe. Biochem. Mosc. 2017, 82, 237-242. [CrossRef]

132. Chan, J.P.; Cordeira, J.; Calderon, G.A.; Iyer, L.K.; Rios, M. Depletion of central BDNF in mice impedes terminal differentiation of new granule neurons in the adult hippocampus. Mol. Cell. Neurosci. 2008, 39, 372-383. [CrossRef] [PubMed]

133. Reichardt, L.F. Neurotrophin-Regulated Signalling Pathways. Philos. Trans. Biol. Sci. 2006, 361, $1545-1564$. [CrossRef]

134. Duman, R.S.; Voleti, B. Signaling pathways underlying the pathophysiology and treatment of depression: Novel mechanisms for rapid-acting agents. Trends Neurosci. 2012, 35, 47-56. [CrossRef] [PubMed]

135. Carlezon, W.A.; Duman, R.S.; Nestler, E.J. The many faces of CREB. Trends Neurosci. 2005, $28,436-445$. [CrossRef]

136. Öngür, D.; Drevets, W.C.; Price, J.L. Glial reduction in the subgenual prefrontal cortex in mood disorders. Proc. Natl. Acad. Sci. USA 1998, 95, 13290-13295. [CrossRef]

137. Bowley, M.P.; Drevets, W.C.; Öngür, D.; Price, J.L. Low glial numbers in the amygdala in major depressive disorder. Biol. Psychiatry 2002, 52, 404-412. [CrossRef]

138. Vostrikov, V.M.; Uranova, N.A.; Orlovskaya, D.D. Deficit of perineuronal oligodendrocytes in the prefrontal cortex in schizophrenia and mood disorders. Schizophr. Res. 2007, 94, 273-280. [CrossRef]

139. Stockmeier, C.A.; Mahajan, G.J.; Konick, L.C.; Overholser, J.C.; Jurjus, G.J.; Meltzer, H.Y.; Uylings, H.B.; Friedman, L.; Rajkowska, G. Cellular changes in the postmortem hippocampus in major depression. Biol. Psychiatry 2004, 56, 640-650. [CrossRef]

140. Rajkowska, G.; Miguel-Hidalgo, J.J.; Dubey, P.; Stockmeier, C.A.; Krishnan, K.R. Prominent reduction in pyramidal neurons density in the orbitofrontal cortex of elderly depressed patients. Biol. Psychiatry 2005, 58, 297-306. [CrossRef]

141. Airan, R.D.; Meltzer, L.A.; Roy, M.; Gong, Y.; Chen, H.; Deisseroth, K. High-Speed Imaging Reveals Neurophysiological Links to Behavior in an Animal Model of Depression. Science 2007, 317, 819. [CrossRef]

142. Srejic, L.R.; Hamani, C.; Hutchison, W.D. High-frequency stimulation of the medial prefrontal cortex decreases cellular firing in the dorsal raphe. Eur. J. Neurosci. 2015, 41, 1219-1226. [CrossRef] [PubMed]

143. Celada, P.; Puig, M.; Casanovas, J.; Guillazo, G.; Artigas, F. Control of Dorsal Raphe Serotonergic Neurons by the Medial Prefrontal Cortex: Involvement of Serotonin-1A, GABA sub(A), and Glutamate Receptors. J. Neurosci. 2001, 21, 9917-9929. [CrossRef]

144. Nambu, A.; Tokuno, H.; Takada, M. Functional significance of the cortico-subthalamo-pallidal 'hyperdirect' pathway. Neurosci. Res. 2002, 43, 111-117. [CrossRef] 
145. Anderson, R.J.; Frye, M.A.; Abulseoud, O.A.; Lee, K.H.; McGillivray, J.A.; Berk, M.; Tye, S.J. Deep brain stimulation for treatment-resistant depression: Efficacy, safety and mechanisms of action. Neurosci. Biobehav. Rev. 2012, 36, 1920-1933. [CrossRef]

146. Deeb, W.; Giordano, J.J.; Rossi, P.J.; Mogilner, A.Y.; Gunduz, A.; Judy, J.W.; Klassen, B.T.; Butson, C.R.; Van Horne, C.; Deny, D.; et al. Proceedings of the Fourth Annual Deep Brain Stimulation Think Tank: A Review of Emerging Issues and Technologies. Front. Integr. Neurosci. 2016, 10, 38. [CrossRef]

147. Schermer, M. Ethical Issues in Deep Brain Stimulation. Front. Integr. Neurosci. 2011, 5. [CrossRef]

148. Bell, E.; Mathieu, G.; Racine, E. Preparing the ethical future of deep brain stimulation. Surg. Neurol. 2009, 72, 577. [CrossRef]

(C) 2020 by the authors. Licensee MDPI, Basel, Switzerland. This article is an open access article distributed under the terms and conditions of the Creative Commons Attribution (CC BY) license (http://creativecommons.org/licenses/by/4.0/). 